NBER WORKING PAPER SERIES

\title{
QUALITY AND ACCOUNTABILITY IN HEALTHCARE DELIVERY: AUDIT-STUDY EVIDENCE FROM PRIMARY CARE IN INDIA
}

\author{
Jishnu Das \\ Alaka Holla \\ Aakash Mohpal \\ Karthik Muralidharan \\ Working Paper 21405 \\ http://www.nber.org/papers/w21405 \\ NATIONAL BUREAU OF ECONOMIC RESEARCH \\ 1050 Massachusetts Avenue \\ Cambridge, MA 02138 \\ July 2015
}

We are especially grateful to Michael Kremer for his involvement as a collaborator in the early stages of this project and for subsequent discussions. We thank Brian Chan, Veena Das, Ranendra Kumar Das, Diana Tabak and Manoj Mohanan for their contribution to designing and implementing the SP methodology, and thank Eun-Young Shim for her contribution to the theoretical appendix. We thank Prashant Bharadwaj, Gordon Dahl, Roger Gordon, Gordon Hanson, and Paul Niehaus for comments. We are grateful to Innovations for Poverty Action, CT, and Pratap Bhanu Mehta and the Center for Policy Research, New Delhi for hosting the project and providing logistical support and infrastructure. We also thank Sreela Dasgupta, L. Ravi and Anvesha Khandelwal for project management support, and Monisha Ashok, Carl Liebersohn, Prerna Mukharya, Suzanne Plant and Anand Shukla for excellent research assistance. The project would not have been possible without the dedication of our SPs and Purshottam, Rajan Singh, Devender, Charu Nanda, Simi Bajaj, Geeta and other staff at Institute of Socioeconomic Research on Democracy and Development (ISERDD) in Delhi. The Social and Rural Research Institute (SRI), New Delhi oversaw data collection of all other aspects other than SPs. This study was funded by the Global Health Program of the Bill and Melinda Gates Foundation through Grant No. 50728 and the Knowledge for Change Program at the World Bank. The findings, interpretations and conclusions expressed in this article are those of the authors and do not necessarily represent the views of the World Bank, its executive directors, the governments they represent, or the National Bureau of Economic Research.

NBER working papers are circulated for discussion and comment purposes. They have not been peerreviewed or been subject to the review by the NBER Board of Directors that accompanies official NBER publications.

(C) 2015 by Jishnu Das, Alaka Holla, Aakash Mohpal, and Karthik Muralidharan. All rights reserved. Short sections of text, not to exceed two paragraphs, may be quoted without explicit permission provided that full credit, including (C) notice, is given to the source. 
Quality and Accountability in Healthcare Delivery: Audit-Study Evidence from Primary Care in India Jishnu Das, Alaka Holla, Aakash Mohpal, and Karthik Muralidharan NBER Working Paper No. 21405

July 2015

JEL No. D40,H10,H42,I11,O15

\begin{abstract}
We present the first direct evidence on the relative quality of public and private healthcare in a lowincome setting, using a unique set of audit studies. We sent standardized (fake) patients to rural primary care providers in the Indian state of Madhya Pradesh, and recorded the quality of care provided and prices charged in each interaction. We report three main findings. First, most private providers lacked formal medical training, but they spent more time with patients and completed more essential checklist items than public providers, and were equally likely to provide a correct treatment. Second, we compare the performance of qualified public doctors across their public and private practices, and find that the same doctors exerted higher effort and were more likely to provide a correct treatment in their private practices. Third, in the private sector, we find that prices charged are positively correlated with provider effort and correct treatment, but also with unnecessary treatments. In the public sector, we find no correlation between provider salaries and any measure of quality. We develop a simple theoretical framework to interpret our results and show that in settings with low levels of effort in the public sector, the benefits of higher diagnostic effort in the private sector may outweigh the costs of market incentives to over treat. These differences in provider effort may partly explain the dominant market share of fee-charging private providers even in the presence of a system of free public healthcare.
\end{abstract}

Jishnu Das

The World Bank

jdas1@worldbank.org

Alaka Holla

World Bank

alaka.holla@gmail.com
Aakash Mohpal

University of Michigan

amohpal@umich.edu

Karthik Muralidharan

Department of Economics, 0508

University of California, San Diego

9500 Gilman Drive

La Jolla, CA 92093-0508

and NBER

kamurali@ucsd.edu 
"It is the general social consensus, clearly, that the laissez-faire solution for medicine is intolerable."

- Kenneth J. Arrow (1963)

\section{Introduction}

Healthcare is a credence good with substantial information asymmetries between patients and providers. This makes it difficult for patients to determine the quality of care they have received. It is widely believed therefore that unregulated market-based delivery of healthcare is socially undesirable (Arrow, 1963). Further, if optimal care requires the potential denial of services that patients value (such as steroids or antibiotics), market-based healthcare may be over-responsive to demand, leading to socially inefficient provision (Prendergast, 2003). Partly as a result of these considerations, the default policy approach to delivering healthcare for the poor in most low-income countries is through free or nominally priced medical care in publicly-run facilities staffed by qualified doctors and nurses, who are paid a fixed salary (World Bank, 2003).

However, for primary care services, a majority of households in low-income countries choose to visit fee-charging healthcare providers in the private sector, whose market share exceeds 70 percent in rural India (the focus of our study). ${ }^{1}$ This is surprising for two reasons. First, private healthcare providers in India face little de facto regulation and most have no formal medical training (Rohde and Viswanathan, 1995; Banerjee, Deaton and Duflo, 2004; CPR, 2011). Second, while the high use of the private sector could, in part, reflect the absence of public options, this cannot be the only explanation. In our data from rural India, the private sector share of primary care visits (constructed from a household census) is 80 percent even in markets with a qualified public doctor offering free care through public clinics, with more than 60 percent of the visits to private providers with no formal qualifications.

The high market share of unqualified private healthcare providers raises a number of questions about the functioning of healthcare markets in low-income settings. First, why would people choose to pay for care from (mostly) unqualified providers when public clinics are staffed with qualified doctors who offer care at a much lower price? Second, how does the quality of care received vary across public and private healthcare providers? Third, what does an unregulated healthcare market reward and how does this compare with the regulated public sector? Specifically, to what extent are prices in the market and wages in the public sector correlated with quality of care? Answers to these questions have been limited by the

\footnotetext{
${ }^{1}$ The market share of private providers is high in many low-income countries: Data from the DHS show that 50 percent of households seeking pediatric outpatient care in Africa and 70-80 percent in India visit the private sector with little variation over the 20 years that these surveys have been collected (DHS, 2007; Grepin, 2014). The World Health Surveys include adult morbidity and here, the numbers vary from 30 percent in Sub-Saharan Africa to between 70 and 80 percent in India (Wagstaff, 2013).
} 
lack of evidence on the actual quality of care provided in public and private health facilities in low-income settings. ${ }^{2}$

This paper uses data from an audit study conducted in rural areas of the Indian state of Madhya Pradesh (MP) to address this gap. Specifically, standardized (fake) patients (SPs) were coached to accurately present symptoms for three different conditions - unstable angina, asthma, and dysentery in a child (who is at home) - to multiple healthcare providers. SPs then made over 1,100 unannounced visits to public and private providers of primary healthcare services and recorded condition-specific metrics of quality of care provided for each interaction, as well as the price charged. ${ }^{3}$ The quality of care metrics include the providers' adherence to a checklist of questions and examinations deemed essential for making a correct diagnosis in each case, their likelihood of pronouncing a correct diagnosis, and the appropriateness of the treatments.

We present results from two sets of comparisons. First, we sent SPs to a (nearly) representative sample of public and private health facilities on a walk-in basis, and we use these data to compare the typical patient experience across public and private clinics. However, these differences reflect variation in both provider composition, as well as differential incentives across public and private clinics. To isolate the effect of practicing in the private sector holding provider characteristics constant, we identified the private practices of qualified public doctors (the majority of whom have one) and sent SPs to present the same medical case to the same set of doctors in both their public and private practices. Our second comparison uses this "dual practice sample" and compares the quality of care across the public and private practices of the same doctors on the same set of cases.

We report three main findings. First, while the majority of private providers in the representative sample have no medical qualifications, they exerted significantly higher effort than public providers and performed no worse on diagnosis and treatment. Private providers spent 1.5 minutes more with patients (62 percent more) and completed 7.4 percentage point more items on a checklist of essential history and examination items (47 percent more) than public providers. They were equally likely to pronounce a correct diagnosis (only 4 percent of public providers do so), to offer a correct treatment (27 percent of public providers do so),

\footnotetext{
${ }^{2}$ Earlier work has highlighted the problem of low doctor effort in the public sector (high absence, low time spent with patients) and low training in the private sector (Banerjee, Deaton and Duflo (2004); Chaudhury et al. (2006); Das and Hammer (2007)). The key evidence gap, however, is the lack of credible estimates of the actual quality of care provided in the public and private sector. For instance, Coarasa, Das and Gummerson (2014) examine 182 cited studies in two systematic reviews of the medical literature and find only one study that adjusts for differences in patients using an audit methodology (as we do here), and no study that adjusts for differences in providers across public and private practices (which we also do here).

${ }^{3}$ Typically used in medical education, SPs are coached to consistently portray a medical case and all of its physical and pyscho-social aspects. When used to evaluate care in hospitals and clinics, they are also trained to accurately recall all aspects of their interactions with the provider. See details in section 3.
} 
and to offer clinically unnecessary treatments (provided by 70 percent of public providers). These differences are not explained by the public sector having high patient loads and waiting times, or inadequate equipment and facilities, and the results hold even after controlling for these factors, and including market fixed effects.

Second, in the dual practice sample the same doctors spent more time with SPs, completed more items on the checklist, and were also more likely to offer a correct treatment in their private practices, relative to their public practices. Notably, we do not find evidence of differential over-treatment under market incentives, with equivalently high rates of unnecessary treatments, use of antibiotics, and total number of medicines in both types of practices. These differences are conditional on seeing the doctor and therefore understate the difference in the quality of patient experiences across public and private practices of the same doctor, because the expected number of trips to the clinic to see a qualified doctor is considerably higher in the public practice (due to high absence rates).

Third, we find a positive correlation between the fees charged by private providers and measures of quality such as the time spent, the fraction of checklist items completed, and likelihood of providing a correct treatment. However, we also find a positive correlation between prices and the total number of medications given - including unnecessary treatment. In the public clinics, SPs were provided free or nominally priced care. Since there is no variation in prices, we examine the correlation between doctors' compensation and quality of care and find no correlation between salaries (or desirability of posting) in the public sector and any measure of quality of care delivered.

To help interpret our results, we develop a simple theoretical framework that models provider-patient interactions in two stages: consultation and treatment. Patients present their initial symptoms to the provider, based on which he forms a prior distribution regarding the true ailment. Higher effort in the consultation stage yields a more precise posterior distribution. The treatment choice is determined by a combination of the physician's desire to cure the patient, market incentives for over-treatment, and patients' demand for medication. The main insight of the model is that while providers will typically exert more effort in their private practice, the effect on overall patient health is ambiguous. If the default effort level of doctors under low-powered incentives is reasonably high, the marginal gains from additional effort in private practice are outweighed by the costs of over-treatment resulting from market incentives. On the other hand, if the default effort level is low, the benefits of higher effort in the private sector (and the resulting increase in precision of the posterior) may outweigh the costs of over-treatment under market incentives. The increase in diagnostic precision from extra effort may also help explain why we do not find higher levels of over-treatment in the private sector in the dual sample, though we find a positive marginal incentive to over-treat. 
Our methodological contribution helps address the fundamental problem of inferring quality in healthcare, where the optimal action is patient and condition specific, and inefficiencies include under-treatment, over-treatment, or both (Pauly, 1980). Specifically, there are four advantages to the use of unannounced SPs relative to existing measures in the literature, which are based on tests of provider knowledge, or observation of medical practices.

First, the use of SPs ensures a common set of patient and illness characteristics, which limits concerns about differential patient sorting across clinics on the basis of personal or illness characteristics, as might be the case when observing real patient-provider interactions. Second, the SP method allows us to objectively score the quality of care using conditionspecific metrics (checklist completion, diagnosis, and treatment) because we know the actual illness being presented and the optimal care associated with the case. In the case of real observations, we would observe only the presenting symptoms and would have to speculate about the true underlying illness. ${ }^{4}$ Third, we are able to observe prices charged for completed transactions, which allows us to study the extent to which the unregulated market rewards quality, and improves upon audit studies in other settings that obtain price quotes but do not complete the purchase. ${ }^{5}$ Finally, Hawthorne effects are not a concern in the SP context because providers do not know that they are being observed. ${ }^{6}$

Substantively, the advances in measurement above combined with our ability to observe the same doctor across public and private practices allow us to provide the first direct comparison of the quality of care across public and private sectors. We also provide the first evidence on how market prices for healthcare behave in an unregulated setting and show that there is a positive correlation between price and checklist completion (and correct treatment), but also between price and unnecessary treatments. This suggests that unregulated market prices do reflect some information on the quality of care, but also that patients cannot evaluate whether they are being over-treated and charged for unnecessary treatments.

These findings are consistent with the broader empirical literature on credence goods that has demonstrated over-provision of services to the detriment of customer welfare in settings

\footnotetext{
${ }^{4}$ Medical vignettes, which measure provider knowledge, also allow for standardization of case-mix and knowledge of the actual illness underlying the presented symptoms, but do not measure actual provider practice, which has been shown to differ markedly from provider knowledge in multiple contexts (Rethans et al., 1991; Leonard and Masatu, 2005; Das and Hammer, 2007).

${ }^{5}$ For instance, first price offers can be very different from the price of the completed transaction if the distribution of willingness to pay is different across populations. See for instance, Ayres and Siegelman (1995) and Goldberg (1996) for an example of how the lack of completed sales data can lead to misleading conclusions in audit studies of car sales. In our case, the "sale" is always completed as the SP leaves only after the provider has completed the interaction and the price has been paid.

${ }^{6}$ The main limitation of the SP method is that only a few types of cases can be presented. To test whether our SP results are externally valid, we also observed the providers in our sample during a typical day's practice, and found very similar results across all their patient interactions (see section 7.1).
} 
ranging from caesarian sections to car mechanics and cab rides for tourists (Wolinsky, 1993; Gruber and Owings, 1996; Dulleck and Kerschbamer, 2006; Dulleck, Kerschbamer and Sutter, 2011; Schneider, 2012). However, as is well known, inefficiencies in market provision do not imply that public provision will do better, and a key contribution of our paper is the ability to compare public and private provision of a canonical credence good such as healthcare.

Combined with the theoretical framework, our results suggest that in settings of poor governance and administrative accountability in the delivery of primary healthcare services through the public sector (Banerjee, Deaton and Duflo, 2004; Banerjee, Duflo and Glennerster, 2008), market-based provision of healthcare may present a legitimate alternative in spite of its many theoretical (and empirical) weaknesses. Further, while public healthcare is free to the consumer, it is not free to the taxpayer. We calculate the per-patient cost in the public sector and conservatively estimate it to be four times higher than the fees charged by private providers in our sample. Thus, the unregulated private market for healthcare, which is mainly staffed by unqualified providers, appears to deliver higher provider effort and comparable quality of care, at a much lower cost per patient. Our results have direct implications for global policy debates on the organization and delivery of healthcare services in low-income countries with low state capacity to deliver effective oversight over public healthcare systems. We discuss these along with caveats in the conclusion.

The rest of this paper is organized as follows. Section 2 describes healthcare provision in rural India and Madhya Pradesh; section 3 describes the standardized patient (SP) methodology, sampling, data, and measures of healthcare quality; section 4 presents a theoretical framework to interpret our results; section 5 presents results on quality of care; section 6 covers pricing and cost-effectiveness; section 7 discusses robustness to alternative explanations; and section 8 concludes with a discussion of policy implications and caveats.

\section{Context}

\subsection{Healthcare in Rural India}

Healthcare in India is delivered by both public and private clinics and hospitals. In the public sector, patients can obtain primary care on a walk-in basis in facilities differentiated by their level of specialization ranging from district hospitals and Community Health Centers (CHCs) to Public Health Centers (PHCs) and sub-centers. ${ }^{7}$ PHCs, CHCs, and hospitals are supposed to be staffed with trained doctors, who are expected to make diagnoses and either treat or refer patients as appropriate. Sub-centers are supposed to be staffed with qualified

\footnotetext{
${ }^{7}$ Official guidelines stipulate that there should be a sub-center for every 5,000 people, a primary healthcare center for every 25,000 people, and a community health center for every 100,000 people.
} 
nurses with doctors visiting on a fixed rotation. Most doctors hold a Bachelor of Medicine and Bachelor of Surgery (MBBS) degree, the rough equivalent of an MD in the US, and receive a fixed salary from the government, with no variable compensation based on either patient load or quality of care. ${ }^{8}$

Consultations in public clinics are provided on a walk-in basis during opening hours (appointments are rarely used), and are free or nominally priced. Patients are also supposed to receive free medication, if available. Although a federally-funded insurance program for inpatient hospital care was introduced in 2007, the tax-funded public system of care was the only source of (implicit) public insurance for primary care.

In theory, public facilities are accountable to administrative norms and procedures (documented in the Civil Service Codes for each state). In practice, administrative accountability of public health-care providers is weak. Nationwide, doctor absences in public clinics averaged 43 percent on any given day in 2003 and 40 percent in 2010 (Muralidharan et al., 2011; CPR, 2011). These absences do not occur on predictable days or hours (Banerjee, Deaton and Duflo, 2004) and they are not easy to address at a system-level (Banerjee, Duflo and Glennerster, 2008; Hanna and Dhaliwal, 2015). When asked about adherence to administrative rules, more than 80 percent of public sector doctors agree that the rules and norms are frequently flouted and that appropriate 'payments' can allow providers to circumvent disciplinary proceedings, even for grave negligence (La Forgia and Nagpal, 2014).

While official policy documents of the Government mainly focus on improving the public system of primary healthcare (Planning Commission of India, 2013), data from household surveys consistently show that the fee-charging private sector accounts for over 70 percent of primary care visits (DHS, 2007; Selvaraj and Karan, 2009; CPR, 2011). Barriers to entry for private healthcare providers are low. Provider qualifications range from MBBS degrees to no medical training at all, and clinics can range from well-equipped structures to small one-room shops, the provider's residence, or the patients' home for providers that make home visits. Providers operate on a fee-for-service basis, and prices often include the cost of medicines. While providers operating without a medical license are not legal and face the threat of being shut down, they have come to be the dominant source of care in these markets (as the data below will show).

\footnotetext{
${ }^{8}$ India also recognizes medical degrees from alternative schools of medicine including the BAMS (Bachelors in Ayurvedic Medical Sciences), the BHMS (Bachelor of Homeopathic Medical Sciences) and the BUMS (Bachelor of Unani Medical Sciences). However, providers with these qualifications are only licensed to prescribe medication in line with their training and are not given prescription rights on allopathic medicine. They also are not typically posted in the frontline healthcare system of PHCs, CHCs, and district hospitals that prescribe allopathic medicine.
} 


\subsection{Sampling of Healthcare Markets, and Summary Statistics}

We carried out the SP study in the Indian state of Madhya Pradesh (MP), one of India's poorer states, with a GDP/capita of $\sim \$ 600 /$ year (or $\sim \$ 1500 /$ year in PPP terms) in 2010 11 (the period of the study). We first drew a representative sample of 100 villages across 5 districts, stratified by geographic region and an index of health outcomes. We then conducted a household census in these villages, where respondents named all providers from whom they sought primary care in the past thirty days and their locations (including providers outside the village, but in market clusters on the nearest main road). We then surveyed all providers in all of these locations, regardless of whether or not the providers themselves had been mentioned in the sample villages, thereby obtaining a census of all providers in the healthcare market that catered to sampled villages.

Table 1 (columns 1-3) presents summary statistics based on the provider census (Panel A) and the household census (Panel B) in these markets; columns 4-6 compare villages sampled for the SP study to the representative villages. The table highlights three key features of health markets in rural India. First, villages are served by a large number of providers once the health market is correctly accounted for by including locations that are near but outside the village. There are 11 primary care providers per market and 46 percent of households reported visiting a primary care provider in the 30 days prior to the survey.

Second, the majority of providers are private ( 7 out of 11 or 64 percent), and they account for 89 percent of household visits; excluding paramedical public health workers (typically responsible for preventive, maternity and child care) increases the fraction further to 93 percent. The share of visits to private providers (with or without qualifications) is 88 percent when there is a public provider in the market, and is 83 percent even when there is a public MBBS doctor in the same market.

Third, 48 percent of all providers and 77 percent of all private providers ( 5.4 per village) have no formal medical training, but account for 77 percent of household visits. There is less than one MBBS doctor per market, and they are rarely available within the village. The distribution of MBBS providers is uneven. Only 30 percent of all villages have recourse to an MBBS provider (public or private) in their market, and only 5 percent within village boundaries. Private unqualified providers remain the dominant providers of care in most settings, accounting for 74 percent of all visits even when there is a public provider in the same market, and 60 percent even when there is a public MBBS doctor in the same market. ${ }^{9}$ MBBS doctors account for only 4 percent of all patient interactions (Panel B).

\footnotetext{
${ }^{9}$ Note that even public facilities have many unqualified providers. While these are typically support staff (who are only supposed to assist a qualified doctor), we find that it is very common for these staff to act as the main healthcare providers in public clinics and prescribe medication (given high doctor absence rates).
} 


\section{Measuring Healthcare Quality Using Standardized Patients}

\subsection{The Standardized Patient (SP) Methodology}

Used routinely in the training and evaluation of medical students in high-income countries, including the United States, SPs are highly-trained 'fake patients' who present symptoms of an illness to a physician like any other normal patient. Details of the SP interaction can be used to evaluate the quality of care received by a typical patient (Rethans et al., 1991). SPs are coached to present their initial symptoms and answer any questions that the physician may ask as part of history taking, in a manner consistent with the underlying condition. We followed the same method (adapted to local conditions) and sent unannounced SPs to healthcare providers in our sample during the course of a normal working day.

A total of 22 SPs were recruited from the districts where the study was conducted. Using a team comprising of a professional SP trainer, two medical doctors, and a medical anthropologist familiar with local forms of presenting symptoms and illnesses, SPs were coached to accurately and consistently present one of three cases - unstable angina in a 45 year-old male, asthma in a 25 year-old female or male, and dysentery in a child who was at home presented by the father of the child (see Das et al. (2012) and Appendix B for details on SP protocols). ${ }^{10}$ SPs visited sampled providers, who did not know they were receiving standardized patients and therefore should have treated them as new patients. ${ }^{11}$ After the interaction, SPs were debriefed within an hour with a structured questionnaire that documented the questions and examinations that the provider completed or recommended, the treatments provided, and any diagnoses offered. The SPs retained any medicines dispensed in the clinic and paid all fees charged by providers at the end of the interaction.

The SPs depicted uncomplicated textbook presentations of the cases, and a panel of doctors who advised the project concurred that appropriate history taking and examinations should lead providers towards the correct diagnosis and treatment. Cases were specifically chosen so that the opening statement by the SPs would be consistent with multiple underlying illnesses, but further questioning should have led to an unambiguous (correct) diagnosis. This allows us to measure provider quality through adherence to an essential checklist of questions and examinations that would allow them to accurately make a diagnosis and pro-

\footnotetext{
${ }^{10}$ Das et al. (2012) discusses the SP methodology in further detail, and presents summary statistics on overall quality of care in this setting. The current paper focuses on the economics of unregulated healthcare markets and we do not replicate the analysis in Das et al. (2012). See Appendix B for further details on how the SP method was implemented, including further discussion on the choice of cases and their relevance. Details on case presentations and instruments are posted on www.healthandeducationinindia.org

${ }^{11}$ The research ethics board of Innovations for Poverty Action approved this design following a successful pilot in Delhi, where the detection rate of SP's was extremely low even among a set of doctors who were informed that they would receive an SP at some point in the next month.
} 
vide a correct treatment. We also chose these cases since they represented conditions with high or growing incidence in India and other middle- and low-income countries, and minimized risk to SPs that could arise from unsafe invasive examinations, such as a blood test with an unsterilized needle.

In these cases the role of suitable medical advice was important because real patients would be unlikely to be able to categorize the symptoms as "life threatening" or "potentially non-harmful" and triage themselves into clinics or hospitals. For instance, the SP with unstable angina complains of chest pain which, even in countries with advanced health systems, is often mistaken by patients as arising from heartburn, exertion or muscle strain. ${ }^{12}$ Similarly, wheezing and shortness of breath in asthma may arise from short-term allergies to environmental contaminants. Finally, for any child with diarrhea, a key contribution of a healthcare provider is to assess whether the symptoms reflect a bacterial or viral infection (and thus whether the patient requires antibiotics) and the degree of dehydration - each of which may be difficult for parents to assess.

\subsection{Healthcare Provider Sampling and Summary Statistics}

Our study first uses the census of healthcare providers described earlier to construct a near representative sample of public and private healthcare providers in rural MP in three of the five sampled districts. While our SPs were recruited from the districts in our sample, they were never residents of the villages where they presented themselves to health providers. Since providers in rural areas might know their patients, the SPs had to justify their presence in the area by mentioning, for example, work-related travel or visits to relatives. For such excuses to be plausible, our final sample dropped villages that could not be accessed by paved roads and comprised of a total of 46 villages across three districts. While these sampled villages have more providers on average than the entire representative set of villages, there is no difference in the composition of providers across the frame and sample (Table 1).

Since SPs visited clinics to obtain primary care, we excluded community health workers, midwives, and providers that only made home visits. We then sampled all public clinics (some large ones were sampled twice), and a maximum of six private providers in each market for a total of 235 clinics, and SPs completed interactions with 224 providers. ${ }^{13}$

Data from this 'representative sample' allow us to compare care provided across typical public and private clinics in rural MP (all estimates are re-weighted by the inverse of the

\footnotetext{
${ }^{12}$ The REACT study in the United States found that many chest pain patients delayed calling 911 because they confused their symptoms with heartburn (Faxon and Lenfant, 2001).

${ }^{13}$ In one case, a sampled village was near a market with over a hundred different healthcare providers. In this one case, we sampled over 20 private providers. See Appendix A for further details on sampling.
} 
sampling probabilities to provide population representative averages). However, this comparison would reflect a combination of varying composition of providers across public and private clinics, as well as the effect of practicing in the private sector.

To isolate the role of private sector practice, we identified the universe of public MBBS doctors posted to PHCs and CHCs from all five study districts, even if these clinics were not located in the village-based sampling scheme. We then identified the private practices of these doctors (we found a private practice for 61 percent). We sampled and successfully administered SP visits to 118 public MBBS doctors. Our 'dual sample' consists of the 88 doctors in this MBBS sample who also have a private practice, and for 69 of these, SPs presented cases in both their public and private practices. The 'dual sample' enables a comparison of the quality of care provided by the same doctor on the same case across his public and private practices. SP completion rates in the dual sample were higher in the private (93 percent) compared to public practices (75 percent), due to higher doctor absence rates in their public practice, leading to non-completion despite multiple attempts. If doctors who are more absent also provide lower quality care in their public sector practice, our public-private differences may be under-estimated.

Note that in the representative sample, the unit of analysis is the clinic and the SP experience is recorded based on whoever they saw in the clinic. In the dual sample, the unit of analysis is the doctor and the SP made repeat visits to see the sampled doctor if needed (especially in the public practice). Appendix A and Tables A.1 and A.2 provide further details on the sampling and construction of the representative and dual samples.

Table 2 (columns 1-3) provides summary statistics for the representative sample of providers. The providers are mostly middle-aged men and just under 60 percent have completed 12 or more years of education (Table 2, Panel A). Their practices have been open for 13-15 years, and private and public providers self-report an average of 16 and 28 patients per day, respectively. Most practices (82 percent of private and 100 percent of public) dispense medicines in the clinic itself and are equipped with the infrastructure and medical devices required for routine examinations, such as stethoscopes and blood pressure cuffs. In the representative sample, public providers are more likely to have an MBBS degrees (26 percent vs. 8 percent). Private providers charged an average of Rs.51 per interaction. Consistent with nominally priced public care, our SPs paid Rs.3.7 on average in public clinics.

Column 4 presents summary statistics on the universe of public MBBS doctors, while columns 5-7 present these for the 88 public MBBS doctors in the dual sample and test if they are comparable. Overall, doctors with and without dual practices are similar on observable characteristics, but the former have a longer tenure at their current location. There is no significant difference in the equipment reported across these practices (Columns 
8-10), although the overall number of patients seen is higher in the public practice and the fees charged are higher in the private practice.

We randomly assigned three SPs to each sampled clinic in the representative sample, one presenting each of the three cases. For the dual sample, we sent SPs presenting the asthma and dysentery cases to both practices of the same provider. ${ }^{14}$ Since the rarity of unstable angina could raise suspicions if providers saw two travelers presenting the same case (even though visits were typically separated by a few weeks), we randomized the providers into two groups - one that received an unstable angina patient in his/her private practice and another that received the case in the public clinic. We show that the randomization was valid in Table A.3.

\subsection{Measuring Quality of Care}

We use three measures of quality of care. Our first metric is the extent to which the provider adhered to a checklist of questions and examinations required for making a differential diagnosis on each of the presented cases. For instance, these questions and exams would allow a doctor to distinguish between heartburn (that has gastrointestinal origins) and a heart attack, or between viral diarrhea and dysentery. These items represent a parsimonious subset of the Indian government's own guidelines, and the list we use was developed by a panel of Indian and American doctors (the items are described for each case in Table A.4). ${ }^{15}$ While the most transparent measure of checklist adherence is the percentage of checklist items completed, we also compute an index score using Item Response Theory (IRT), which gives more weight to items that discriminate better among providers. Developed in the context of educational testing, IRT allows us to create a composite measure of provider quality based on questions asked across all three cases, with lower weights on checklist items that are less essential and higher weights on more essential questions that do a better job of discriminating between low and high quality providers (see Das and Hammer (2005) for details). We

\footnotetext{
${ }^{14}$ Since we had 22 SPs and 3 cases, we made sure that the same case was presented by different SPs in the public and private practices. To ensure that our standardized patients saw the sampled provider when (s)he visited the public clinic and not a substitute, we first interviewed all providers in their private practices or residences without revealing that we knew they also worked in the public sector, and we obtained either their photograph or a detailed description of their physical appearance. SPs portrayed a dummy case (e.g. headache) if the doctor was absent when they visited the public clinic, and we sent in other SPs on our subsequent attempts. As we discuss later, it took significantly more trips to complete an SP case in the public practice relative to the private one, due to the high rates of provider absence in the public practice.

${ }^{15}$ The Indian government's National Rural Health Mission (NRHM) has developed triage, management, and treatment protocols for unstable angina, asthma, and dysentery in public clinics, suggesting clear guidelines for patients presenting with any of these conditions. The checklist we use is more parsimonious than what the Indian government's own guidelines recommend. If we use the more extensive checklist, this would likely deflate the checklist adherence further below the low numbers that we document.
} 
report both measures in our analysis.

Second, we examine diagnoses - whether one was provided and whether it was correct. We consider a diagnosis incorrect when it cannot even be considered partially correct - for example, a provider tells an asthma patient that she has a gastrointestinal problem or an unstable angina patient that the weather is causing his ailment. Our definitions of correct and incorrect diagnoses are presented in Table A.4 - Panel B.

Third, we evaluate the quality of treatment provided. SPs noted all treatment instructions received and retained all prescriptions and medication dispensed in the clinic. These were then classified as correct, palliative, or unnecessary/harmful, based on inputs from our panel of doctors, pharmacists, and a pharmaceutical company (see Appendix B.4 for details; Table A.4 - Panel C lists specific treatments in each category). Since providers can dispense or prescribe multiple medicines, we classify each medicine as correct, palliative, or unnecessary/harmful and thus allow the total treatment protocol to be classified into multiple categories at the same time.

Correct treatment refers to a treatment that is clinically indicated for the specific case and that would relieve/mitigate the underlying condition. Palliative treatments are those that may provide symptomatic relief, or treatments where the providers correctly identified which system was being affected, but which on their own would not cure the patient of the condition that was being presented - for example, allergy medicine for the asthma patient. Treatments classified as unnecessary/harmful were neither correct nor palliative. We group these two potentially distinct categories together because it was difficult to achieve consensus among doctors on what should be considered harmful. Some, for example, would consider antibiotics for the unstable angina patient unnecessary. Others took a longer view with antibiotic resistance in mind and considered it as ultimately harmful. However, none of the treatments we observed were directly contra-indicated, and hence most of these represent unnecessary treatments as opposed to directly harmful ones. ${ }^{16}$

However, even after classifying all medicines as correct, palliative, and unnecessary/harmful, there are two challenges in coding the "correctness" of a treatment. The first is: How should we interpret a referral when incentives are very different? In some cases, this may be a good thing (if, for example, the provider refers a heart attack patient to a hospital). In other cases, a "referral" may simply reflect a provider who deflected the case without directing the

\footnotetext{
${ }^{16}$ If the overall quality of care were higher, we could have designed the SP case with a patient who is allergic to certain kinds of antibiotics or who is on regular medication for another illness. In this case, many treatments would have been harmful and the case would have required the doctor to watch out for drug interactions. Given the low-level of overall quality of care, designing such an SP case would not have been very useful at discriminating quality because SPs were never asked about existing allergies or whether they were currently taking any medication.
} 
patient usefully. ${ }^{17}$ Since we did not send the SPs to the place that was referred, there is no obvious way of coding the quality of referrals. We therefore try to be conservative in our main analysis and do not treat referrals as correct treatments. When we repeat the analysis treating referrals as correct in the angina case, our results are unchanged (results below).

A second challenge arises from the proxy nature of the dysentery case. Many providers did not provide a treatment because the child was not presented and instead asked to see the child. We therefore report results for 'checklist completion' using all three cases, but drop the dysentery case for 'diagnosis' and 'treatment' because the patient (the sick child) was not actually presented for this case. All results are robust to dropping the case completely.

\section{Theoretical Framework}

A simple theoretical framework helps to interpret our results, by characterizing the optimal effort and treatment choices that a provider is likely to make with and without market incentives, and the effects of their choices on patient health outcomes. We present the main insights here, with full derivations in Appendix C. The interaction between doctors and patients is modelled in two stages - consultation and treatment - where providers first engage in (Bayesian) learning about the patient's condition and then treat. A patient enters the clinic and presents her symptoms, based on which the provider forms a prior belief about the underlying disease that caused the symptoms given by:

$$
n^{\text {prior }} \sim N\left(\nu, \frac{1}{\alpha}\right)
$$

The provider, who has medical knowledge, $K$, exerts effort $e$ and draws a signal $s \sim$ $N\left(n^{\text {true }}, \frac{1}{\beta}\right)$, where $n^{\text {true }}$ is the correct underlying state and $\beta=e K$. Providers improve the precision of the signal by either exerting higher effort, or being more knowledgeable, or both.

The provider's posterior belief is then:

$$
n^{p o s t} \sim N\left(\mu, \frac{1}{\alpha+\beta}\right)
$$

where $\mu$ is the posterior mean given by: ${ }^{18}$

\footnotetext{
${ }^{17}$ Field notes suggest that this often happened in public clinics where the doctor was absent. The available provider did not ask questions or conduct any examinations, and told the SP to go elsewhere. By necessity, this is coded as a "referral" in our data, although the patient received no information from the interaction.

${ }^{18}$ Note that the marginal effect of $e$ on posterior precision diminishes as $e$ becomes larger as illustrated in Figure 1 (Panel B). Also, as in Rosenzweig (1995) a doctor with more knowledge may also have a more accurate prior to begin with, in addition to learning more with additional effort. We abstract away from this point to focus on deriving predictions for effort, treatment, and health outcomes for the same doctor across public and private practices. This corresponds to our dual sample.
} 


$$
\mu=\frac{\alpha \nu}{\alpha+\beta}+\frac{\beta s}{\alpha+\beta}
$$

In the second stage, the provider makes treatment choices based on the posterior belief about the true state. The choice of treatments is expressed as an interval $[\mu-n, \mu+n]$, which maps into the empirical observation that most providers in our setting provide multiple medications. A wider range of treatments has a higher probability of covering the true illness and curing the patient of the current ailment but also increases long-term health costs. ${ }^{19}$ The patient's health outcome given $e$ and $n$ is denoted by $H(e, n)=P_{e}(n)-h(n)$, where $P_{e}(n)$ is the probability that $n^{\text {true }}$ is covered by the treatment and $h(n)$ is the health cost which increases with $n$. Thus the optimal outcome for a patient is to receive only the correct treatment, and not receive any additional unnecessary treatments, and we can think of a high-quality provider as someone who provides this outcome, enabled by a precise posterior distribution of the true illness.

In practice, providers will choose effort and treatments to maximize their own utility, which may not be aligned with those of patients. We model provider utility as having three components. First, providers care about curing their patients and overall patient health. This can be attributed partly to altruism, intrinsic motivation to do the right thing, training and professionalism (Hippocratic oath), peer pressure and monitoring, and the liability and malpractice regime. We capture all of these factors with the parameter $\phi$, which should be thought about as representing the extent to which providers value patient health in their utility in a setting without high-powered financial incentives. Thus, a higher $\phi$ represents greater alignment between provider and patient utility.

Second, providers also care about financial rewards, which in turn depends on how they are compensated. Under market pricing, providers can charge a consultation fee $(\tau e)$ that is a function of a piece rate $\tau$ (determined by their qualifications and reputation) and effort expended (which is observable to patients), and a dispensing fee that increases linearly with the number of medicines provided. They also have an incentive for improving patient health because this helps build their reputation and raises future demand (which we can think of as an increase in their consulting piece rate over time). However, patients can observe whether they were "cured" more easily than the costs of excessive medication, and this creates an incentive to over-treat because over-treatment increases the probability of spanning the true illness and providing a correct treatment. We denote the observed health outcome as $H^{o}(e, n)$, and true health as $H(e, n)$.

Third, providers' treatment choice may respond to patient demand. Patients may self-

\footnotetext{
${ }^{19}$ This is a standard assumption in the medical literature and can be motivated by either the building of resistance to unnecessary drugs or by the potential for adverse interactions between drugs.
} 
diagnose their illnesses and demand medications that they think they need, ${ }^{20}$ or may simply seek pain-killers, steroids, and other drugs that provide symptomatic relief but are medically inappropriate for their condition. In such cases, it can be costly for providers to not provide medicines that patients demand, and we model patient-induced demand as a communication cost paid by providers to convince patients about the providers' choice of treatment.

In the absence of market incentives and patient-induced demand, providers optimize over:

$$
\begin{gathered}
V_{1}=\max _{e}\left\{-c(e)+V_{2}(e)\right\} \\
V_{2}(e)=\max _{n}\{\phi H(e, n)\}
\end{gathered}
$$

where $V_{1}$ and $V_{2}(e)$ are the maximized utilities in the consultation and treatment stage, and they choose a corresponding level of effort and treatment. Since there is no marginal incentive for either effort or treatment, these will depend only on $\phi$ and the cost of effort. The provider then chooses $n$ that maximizes $H(e, n)$ in the treatment stage (assuming that medicines are provided free to patients as is the norm in public clinics).

Under market incentives, providers maximize:

$$
\begin{gathered}
V_{1}=\max _{e}\left\{-c(e)+\tau e+V_{2}(e)\right\} \\
V_{2}(e)=\max _{n}\left\{\phi H(e, n)+\delta H^{o}(e, n)+n p\right\}
\end{gathered}
$$

where $\tau$ is a piece-rate consultation fee, $\delta$ represents the extent to which improving patients' current observed health improves the provider's reputation in the market and generates future pay-offs, and $p$ is a per unit profit from $n$. Because the health cost of $n$ is not fully observed in the market but the provider derives pecuniary benefits from $n$, he chooses excessive $n$ where $H(e, n)$ is decreasing in $n$. However, compensation for effort $(\tau e)$ and concern about reputation induces higher effort, which yields a more accurate posterior and increases the probability of spanning the true illness even with a smaller $n$, which pushes towards a smaller $n$. Note also that $n$ is bounded from going to infinity because the costs of excessive medication are observed by patients (albeit imperfectly), and also because doctors place a positive weight $\phi$ on $H(e, n)$, which is decreasing in $n$.

Figures 1 and 2 illustrate the main insights of the model. Market incentives typically lead to higher effort, as shown in panel (A) of Figure 1. When $\phi$ is low, providers choose low levels of effort without other incentives, and the difference in the level of effort with and without market incentives leads to a large difference in the posterior precision (panel (B)). Thus,

\footnotetext{
${ }^{20}$ For instance, Cohen, Dupas and Schaner (2015) show that patients with a fever in Kenya often selfdiagnose themselves as having malaria and try to obtain anti-malaria treatments though these are not medically warranted.
} 
while market compensation provides an incentive to over-treat, it also provides incentives for greater diagnostic effort, which yields a more precise posterior. Since increased posterior precision reduces the benefit of choosing large $n$, it is possible that $n$ may be smaller with market incentives as shown in panel $(\mathrm{C})$. With higher effort leading to a greater probability of providing the correct treatment and a smaller $n$ (due to increased diagnostic precision) the resulting health outcome could be better with market incentives. However, as $\phi$ increases, the default level of effort without market incentives also increases, and the marginal gain from additional effort on posterior precision is lower (panel $(B)$ ). In this case, the benefits of additional effort under market incentives are outweighed by the incentives to prescribe more (Panel (D)). Providers choose larger $n$ with market incentives, and the health outcomes are likely to be worse than the case without market incentives.

Figure 2 summarizes this point and shows that market incentives are likely to lead to worse outcomes in settings with a high $\phi$. This may be typical in high-income countries with better oversight of medical training and practice, which is the context where (Arrow, 1963) is implicitly set. However, in settings with very low $\phi$ as seen in India and other low-income countries - exemplified by high doctor absence rates (Chaudhury et al., 2006) - it is possible that market incentives may lead to better outcomes. ${ }^{21}$

Finally, we also add patient-induced demand to the provider's optimization problem. With this cost, we get $n$ closer to the value which the patient demands, though the cost is lower for providers who exert higher consultation effort (because this effort makes it easier to convince patients that their desired $n$ is not good for them). This mechanism provides a plausible explanation for the high levels of unnecessary treatment we observe among public providers (who have no marginal incentive to do so). ${ }^{22}$

We present our framework formally in Appendix C, where we specify and solve the provider's utility maximization problem with and without market incentives, and show how patient health outcomes vary as a function of $\phi$ and the presence of market incentives. We do not endogenize the dynamic price setting process - because the static framework maps into our data and is adequate to interpret our empirical results. A theoretical extension that provides a way of endogenizing the market incentives is available on request.

\footnotetext{
${ }^{21}$ See Muralidharan and Sundararaman (2011) for an adaptation of the multi-tasking framework of Holmstrom and Milgrom (1991) and Baker (1992) that yields similar insights in the context of performance-linked pay for teachers (showing that outcomes could improve under performance pay if the default level of teacher effort was low, but could worsen if the default level was high). A key difference in our context is that the high-powered incentives do not come from administratively set performance-linked bonuses, but market rewards for effort and reputation.

${ }^{22}$ Note that patient-induced demand is not necessary to explain high levels of unnecessary treatment in public clinics (though it may partly do so). Since a less precise posterior is correlated with giving out more medication, our model predicts that less knowledgeable providers as well as those who put in low effort will give out more medicines.
} 


\section{Results - Quality of Care across Public and Private Providers}

\subsection{Estimation Framework}

Our main interest is in estimating differences in the quality of care that patients received from providers in the public and private sectors. In the representative sample, we estimate:

$$
q_{(i(s c p) m)}=\beta_{0}+\beta_{1} \text { Private }_{i p}+\beta_{2} X_{p}+\delta_{s}+\delta_{c}+\delta_{m}+\epsilon_{i(s c p) m}
$$

where we regress each measure of quality $q$ (checklist completion, diagnosis, and treatment) in interaction $i$ between a standardized patient $s$ presenting case $c$, and a provider $p$ in market $m$ on an indicator for the sector (Private), with $\beta_{1}$ being the term of interest. Since we pool cases and SPs and there may be systematic differences across them, all our specifications include SP and case fixed effects $\left(\delta_{s}\right.$ and $\left.\delta_{c}\right)$. We report three sets of estimates for each quality measure. First, we include only SP and case fixed effects; then we add market fixed effects so that comparisons reflect relative performance in the same market (note that not all markets had both types of providers); finally, we add controls for provider and practice characteristics $X_{p}$, to adjust for observable differences across providers including demographics, reported qualifications, and number of patients waiting during the visit.

While $\beta_{1}$ provides a useful estimate of the differences in quality across public and private providers in a representative sample of providers, it is a composite estimate that includes differences in unobservable provider characteristics, as well as the effect of practicing in the private sector. To isolate the impact of private sector practice, we re-estimate equation 8 in the dual sample that only includes data from the cases where we sent the SPs to the public and private practices of the same MBBS doctor. We report three sets of estimates here as well. First, we include only SP and case fixed effects, ${ }^{23}$ then we add district fixed effects (since the dual practice sample was drawn from the universe of public MBBS doctors practicing in each district rather than the universe of providers practicing in sampled villages, as was the case for the representative sample); finally, we include controls for observable differences across the public and private practices of the doctors.

\footnotetext{
${ }^{23}$ Note that we do not include provider fixed effects since the angina case was not presented in both the public and private practices of the same doctor, and will drop out if we do so. Since the case was randomly allocated across the public and private practices of the doctor and assignment was balanced on measures of quality on the other case (see Table A.3), our estimates will be an unbiased estimate of the average quality difference across the public and private practices of public MBBS doctors. We also estimate equation 8 with provider fixed effects and the results are unchanged (but driven by variation in the asthma case).
} 


\subsection{Completion of Essential Checklist of History Taking and Examinations}

Columns 1-3 in Table 3 present results from estimating equation 8 in the representative sample. Our outcome variable is 'provider effort', measured by consultation length and checklist completion. While the results are similar across the three specifications, we focus our discussion on the estimates in Panel B, because they compare relative performance within the same market (without controlling for provider characteristics), which is the relevant choice set for patients. The base level of effort among representative public providers was low. The average public provider spent 2.4 minutes with the SP in a typical interaction and completed 16 percent of checklist items. Private providers spent 1.5 minutes more per patient and completed 7.4 percentage points more items on the checklist (62 percent and 47 percent more than the public providers respectively). When evaluated on the IRT scaled score, private providers scored 0.61 standard deviations higher. Figure A.1 shows that time spent with the patient is strongly correlated with the number of checklist items completed, which points to the credibility of the SP presenting the case, as more time spent with the patient led to greater checklist completion.

Columns 4-6 repeat the analysis in the dual sample, with similar results. Public MBBS doctors appear to be more productive than the typical public provider in the representative sample (many of whom are unqualified) because they complete a slightly higher fraction of checklist items (18 percent) in 35 percent less time (0.9 minutes less). However, this additional productivity is not used to complete more checklist items in the public practice, but rather to reduce the time spent with patients (1.56 minutes versus 2.4 minutes in the representative sample). In their private practices, the same doctors doubled consultation length, completed 50 percent more checklist items, and scored 0.73 standard deviations higher on the IRT-scaled measure of quality. It is worth comparing these differences with those obtained in interventions that are regarded as highly successful. For instance, Gertler and Vermeersch (2013) look at checklist completion as a result of the introduction of performance pay in Rwanda. They find that performance pay increased checklist completion by 0.13 standard deviations; we find that the difference in checklist completion across public and private practices of the same doctor is over five times larger.

These differences are seen clearly in Figures 3-5. Figure 3 plots the cumulative distribution functions (CDF) of the IRT-score (based on checklist completion) of public and private providers in the representative sample, Figure 4 does so for the dual sample, and Figure 5 pools all four samples together (Figures A.2 - A.4 plot the corresponding distributions). The distribution of checklist completion for private providers first-order stochastically dominates that of the public providers (Figure 3) and the corresponding distribution for the private 
practices of public providers also first-order stochastically dominates that of their public practices (Figure 4). Finally checklist completion is higher for public MBBS doctors than a representative public provider (as would be expected given that the former are more qualified), but it is lower for the public MBBS doctors even relative to a representative sample of private providers (most of whom are unqualified, Figure 5).

Focusing on individual checklist items (Table A.5) shows that private providers in both samples are significantly more likely to perform several items on the checklist on all three cases and are no less likely to perform any of the items (except for one in asthma). In addition to $\beta_{1}$, Table 3 (columns 1-3) also shows that there is no correlation between the possession of any formal medical qualification and checklist completion, suggesting that formal qualifications may be a poor predictor of provider effort.

\subsection{Diagnosis}

Results for diagnosis (Table 4) follow the same format as Table 3 but the dependent variables of interest are whether any diagnosis was given and whether a correct diagnosis was given (both conditional and unconditional on uttering a diagnosis). In the representative sample, 26 percent of public providers offer a diagnosis, of whom only 15 percent offer a correct one. The unconditional probability of a correct diagnosis was only 4 percent.

Private providers in the representative sample are more likely to offer a diagnosis but are not more likely to offer a correct one. The probability of offering a correct diagnosis is higher in the dual practice sample (15 percent vs. 4 percent), which is not surprising since these providers are all trained MBBS doctors. Even among these doctors, however, there is no difference in the rate of correct diagnosis between their public and private practices. Overall, the summary statistics, our price regressions (seen later), and our field work suggest that pronouncing a correct diagnosis (or even just a diagnosis) is not seen by providers (and the market) as being essential in this setting, where patient literacy levels are low.

\subsection{Treatment}

Table 5 reports on several outcomes related to the treatment offered, coded as discussed in section 3.3. The probability of receiving at least one correct treatment from a representative public provider was 21 percent. However, they offered non-indicated treatments at much higher rates, with a 53 percent probability of providing a palliative treatment, and a 74 percent probability of providing an unnecessary treatment. Since the majority of providers provide unnecessary treatments, the probability of receiving only a correct treatment and nothing more is 2.6 percent. We can also examine two potential proxies for over-treatment - 
the rate of antibiotic prescriptions and the total number of medicines provided. Antibiotics were prescribed or dispensed in 26 percent of interactions (though they were not indicated for the asthma and angina cases), and an average of 2 medicines per interaction were dispensed.

In the representative sample, we do not find a significant difference between public and private providers on the probability of providing a correct, palliative, or unnecessary treatment; however, point estimates suggest that private providers have a higher probability of providing both correct and unnecessary treatments. Private providers in the representative sample also provide significantly more medicines (over 3 medicines on average, which is 50 percent greater than the public clinics).

In the dual practice sample, we see that treatments provided in the private practice strictly dominate those provided in the public practice of the same doctor. The rate of correct treatment is 37 percent higher (14 percentage points on a base of 38 percent), the rate of providing a clinically non-indicated palliative treatment is 18 percent lower (11.6 percentage points on a base of 63 percent), and the rate of antibiotic provision is 25 percent lower (12.1 percentage points on a base of 48 percent) in the private relative to the public practice of the same doctor.

\subsection{Knowledge and Effort of Public and Private Providers}

As predicted by the model, there is a strong correlation between higher provider effort and probability of giving a correct treatment (Figure 6). Nevertheless, the results in Tables 3 and 5 suggest that the higher effort exerted by private providers in the representative sample does not translate into better treatment outcomes. A natural explanation is that the representative private provider has a lower level of medical knowledge, but compensates with higher effort, yielding comparable overall levels of treatment accuracy (in line with our theoretical framework). To examine this possibility further, we use the 'discrimination' parameter of each checklist item (as estimated by the IRT-model; see Table A.5), to classify individual items into terciles of low, medium, and high discrimination items. Here, higher discrimination items are those that are more effective at distinguishing provider quality. In the model, these would correspond to questions and exams that enable a provider to construct a more precise posterior distribution (since $\beta=e K$, this can be interpreted as a provider with more knowledge spending the effort more efficiently). ${ }^{24}$

Table A.6 reports the same specifications as in Table 3 but compares public and private providers on checklist completion for different levels of item discrimination. As expected, providers are much less likely to complete high discrimination items on the checklist (consis-

\footnotetext{
${ }^{24}$ The classification of items into terciles of difficulty is done within each case, but the results are robust to classifying the items jointly across all cases as well. The terciles for each item are indicated in Table A.5.
} 
tent with low overall quality of care). In the representative sample, private providers complete 11 percentage points more of the low-discrimination checklist items but are no more likely to complete high-discrimination checklist items. However, doctors in the dual sample are significantly more likely to complete both low and high-discrimination items in their private practice. These results suggest that while the representative private provider does exert more effort, their lower knowledge leads to this effort being directed towards questions that are easy to ask and interpret, and may limit the marginal product of their effort. The results also highlight the importance of using the dual sample for holding provider knowledge and unobservable characteristics constant, and isolating the effect of market incentives on quality of care provided.

\subsection{Robustness of checklist and treatment results}

Our main results pool data across cases to maximize power. For completeness, we also show the results from Tables 3-5 by case (Table A.7). The superior performance of private providers on consultation length and checklist completion is seen in each of the three cases and in both the representative and the dual samples. Consistent with the overall results, private providers in the representative sample do not do better on diagnosis or treatment in any of the individual cases. In the dual sample, MBBS doctors were 14 percentage points more likely to correctly diagnose and 28 percentage points more likely to correctly treat the unstable angina (heart attack) case in their private practice relative to their public practices. In the asthma case, they are 11 percentage points more likely to offer a correct treatment (but this is not statistically significant given the smaller case-specific sample size).

We confirm that the results in Table 5 are robust to alternative definitions of correct treatment. Table A.8 shows the specific treatments offered by case, including referral frequency. Table A.9 shows that the results in Table 5 are robust to treating all referrals as a correct treatment. As discussed earlier, we include the dysentery case for the analysis of checklist completion but exclude it from the analysis of correct diagnosis and treatment because of the large (and differential) fraction of cases where the provider did not provide these and instead asked to see the child (see Table A.8). Since checklist completion may also be censored in such cases, we also present the checklist completion results without the dysentery case and the results of Table 3 continue to hold (Table A.10). We also show the core results with controls for clinic-level infrastructure and facilities (Table A.11), and all the results continue to hold, suggesting that the results are not being driven by differences in facilities and infrastructure across public and private clinics. 


\section{Results - Pricing and Cost Effectiveness}

\subsection{Correlates of Prices Charged among Private Providers}

Table 6 presents correlations between prices charged and our various metrics of healthcare quality in the representative sample, dual sample, and pooled sample. The odd columns present binary correlations, while the even columns present multiple regressions. The market rewards several measures of quality of care including time spent, checklist completion rates, and provision of a correct treatment (Table 6, Columns 1, 3 and 5). On the other hand, there is no price premium for pronouncing a correct diagnosis, and a price penalty for referrals; whether this penalty is optimal (without a penalty, every provider should just refer the patient) or reduces provider incentives to refer patients adequately is unclear. Finally, there is a price premium for dispensing medicines, but not for prescribing them. The price charged is increasing in the total number of medicines dispensed, which may provide incentives for the provision of excessive medication and is consistent with our theoretical framework.

Most of these patterns are repeated in the multiple regressions (Table 6, Columns 2, 4 and 6). Note, however, that correct treatment is no longer rewarded in the multiple regressions. This is likely due to the high correlation between the provision of a correct treatment and the checklist completion rate (Figure 6), and the use of medicines. Thus the market appears to reward observable measures of quality such as time spent, checklist completion, and dispensing medicines (which are correlated with the provision of correct treatment), but patients do not appear to be able to discern whether they received the correct treatment conditioning on these observable measures.

The correlates of pricing observed in Table 6 are in line with those predicted by our modeling framework and point to both strengths and weaknesses of market-based incentives for healthcare provision. On one hand, there appear to be positive incentives for the provision of better quality care (including more effort and providing the correct treatment). On the other hand, the results are consistent with evidence from other settings, which show that markets for credence goods with asymmetric information between providers and customers often reward over-provision to the detriment of customer welfare. Overall, the results suggest that the market rewards providers who "do more", which is correlated with doing more "good" things as well as more "unnecessary" things. ${ }^{25}$

In sharp contrast to the market for private healthcare, the public sector rewards qualifications and age (experience), but there is no correlation between provider wages and any of our measures of quality including the time spent, checklist completion, or correct treatment

\footnotetext{
${ }^{25}$ Note that the results are robust to excluding observations where we were not able to identify the medicines provided and classify them as correct or not (see Table A.12).
} 
(Table 7). Since public employees receive non-pecuniary rewards for better performance through more desirable job postings, we also present correlations between the desirability of a posting and measures of quality and again find that the only significant correlate of a better posting is age - suggesting that the public sector does not reward the quality of care provided by doctors with either more pay or with more desirable job postings. ${ }^{26}$

\subsection{Comparative Cost Effectiveness}

While healthcare in the public sector is free or nominally priced to the user, it is not cost-free to the tax payer. Table A.13 presents estimates of the cost per patient in the public sector, and calculates that the cost per patient interaction is around Rs.240. This is a conservative calculation because it uses only the wage cost in the public sector and does not include any cost of infrastructure, facilities, equipment, medicines or administration. By contrast, the fees charged are the only source of revenue for private providers and hence will cover all operating costs. Thus, even though private providers charge higher consultation rates than public providers (as seen in Table 2), the per-consultation fee of Rs.51 charged by private providers is less than a fourth of the cost of a patient interaction in the public sector. ${ }^{27}$

\section{Robustness}

\subsection{Real Patients}

The use of SPs to measure quality of healthcare presents several advantages over the next best method of clinical observations. However, SPs are limited in the number and types of cases that can be presented. Further, we may worry that the SPs present "off equilibrium" situations in the market that do not extend to its general functioning. We therefore supplemented our data collection after completing the SP modules by conducting day-long clinical observations to code actual provider-patient interactions. We conducted these observations in both the representative and dual samples, and observed a provider in both his/her private and public practices. While we cannot code the actual quality of care from these observations (since we do not observe the underlying illness), we record several observable characteristics of each patient interaction based on over 1000 interactions in both samples.

\footnotetext{
${ }^{26}$ These results are similar to those found in publicly-provided education in India and Pakistan, where teacher salaries increase with qualifications and seniority, but are not correlated with their effectiveness at raising test scores (Muralidharan, 2013; Das and Bau, 2014).

${ }^{27}$ Note that we assume that there is a comparable case mix for primary-health visits across public and private facilities, as is standard in comparative cost effectiveness analysis of this sort. This is also consistent with our data from observing real patients (see section 7 below) where we observe considerable overlap in the symptoms presented across public and private clinics.
} 
Table 8 reports results from estimating Eq. 8 with data from real patient interactions. Private providers spend more time with patients, ask more questions, and are more likely to conduct a physical exam. They also give out more medicines on average. Results from the dual sample are also remarkably similar to those in Tables 3-5, with private providers still exhibiting higher effort but not providing more medicines. Thus, while our SPs present only three specific cases, our results from observing real interactions between patients and providers across the entire set of cases seen in a typical day are very similar to those from the SPs, suggesting that our SP-based results may be valid for a wider range of cases.

\subsection{Statistical Discrimination}

One issue to consider in interpreting our dual-sample results is the possibility that doctors expect to see different patients across their public and private practices, and that the differences we observe do not reflect market incentives as much as statistical discrimination.

We address this concern in three ways. First, we note that the cases are both standard and ubiquitous in our setting. Thus, there is no reason to expect that the optimal treatment protocol should vary by the affluence level of the patient or their ability to afford follow up treatments. Second, we conducted detailed exit interviews with a sample of patients from each clinic that we conducted physician observations in, and find that there aren't many differences on average in patient characteristics across public and private clinics (see Table A.14). Finally, since there are some differences in average patient characteristics across public and private clinics in Table A.14, we construct a propensity score of the likelihood that a patient with a given set of observable characteristics will be seen in a public or private clinic, and show that there is considerable common support in these distributions (Figure A.5). In other words, for the majority of observable characteristics, it is not the case that patients go exclusively to a public or private clinic, suggesting that our results are unlikely to be explained by statistical discrimination (see Appendix D for a more detailed discussion).

\subsection{Strategic Diversion of Effort in the Dual Sample}

A further issue in interpreting our dual-sample results is the possibility that doctors with private practices may deliberately under-provide effort in their free public practices to shift demand to their fee-for-service private practices (see Jayachandran (2014) for a similar example from education). While we cannot fully rule out this possibility, there is suggestive evidence against this. We compare public providers with and without a private practice and find that providers with a private practice are not any more likely to refer away an SP (Table A.15). Providers with a dual practice do provide less effort in their public practices relative 
to those without a private practice, but the lack of any evidence of differences in referral rates suggest that these differences may reflect selection rather than strategic behavior with more publicly conscientious doctors being less likely to have a private practice.

The relevant policy question is whether doctors will start exerting more effort in their public practice if the option of private practice did not exist. But it is worth noting that private practice by public MBBS doctors was illegal in MP during the time of our study, and that over 60 percent of providers still had a private practice, consistent with the idea that this is a low $\phi$ environment.

\subsection{Alternative Comparisons in the Representative Sample}

Finally, our representative sample analysis compares the average public and private provider in a market, but it is not clear if the average is the correct metric for quality since patients can choose the best provider in the market. We therefore present an alternative comparison between the best public and best private provider in each market in Table A.16 and find that our results are very similar to those in Tables 3-5.

\section{Discussion and Conclusion}

Using an audit methodology, we present the first set of results on the quality of public and privately provided healthcare in a low-income country that features a de facto unregulated private sector. Our data suggest that patients in our setting have few good options for healthcare - public or private. Private sector providers, the majority of whom have no formal medical training, spend more time with patients and are more likely to adhere to a checklist of recommended case-specific questions and examinations, but their effectiveness appears to be ultimately limited by their low level of medical knowledge. Public sector clinics, though theoretically staffed by qualified providers, are characterized by lower provider effort. Posts are vacant and doctors are frequently absent, so that even in a public sector clinic, the patient often sees a provider without formal training. The lower effort (compared to the private sector), appears to offset the benefit of more qualified providers in the public sector, and ultimately there is little difference in correct treatment or the overuse of incorrect medicines across a representative sample of public and private providers. Further, our best estimates of cost per patient interaction suggest that the public healthcare system in India spends at least four times more but does not deliver better outcomes than the private sector. ${ }^{28}$

\footnotetext{
${ }^{28}$ These results mirror recent experimental evidence on primary education. Muralidharan and Sundararaman (2015) find that private schools in rural India deliver equal or superior learning outcomes than public schools, even though public schools spend three times more per student. Private school teachers are less qualified than public teachers, but exert much higher levels of effort. Thus, private providers in both primary
} 
Comparing the same provider in the public and private sector allows us to isolate the effect of market-based accountability in the private sector and compare it with administrative accountability in the public sector. The first appears to perform better on all counts. Adherence to checklists and correct treatment rates are higher in the provider's private clinic, and the extent of unnecessary treatments is no different.

These results are consistent with the hedonic earnings-effort relationship in the private sector, which is absent in the public sector. Providers in the private sector earn more when they complete more of the medically necessary checklist and when they provide a correct treatment, showing that the market rewards certain key aspects of high quality. However, the market also rewards unnecessary treatments (consistent with healthcare being a credence good), and patients frequently receive treatments that they do not need, and pay for them.

Despite market incentives for over-treatment, one surprising result is that the rate of provision of unnecessary medication is equally high in the public clinics. Our theoretical framework provides a possible explanation for this result by showing that unnecessary treatments are not only driven by market incentives, but can also arise from low diagnostic effort. In our setting of low $\phi$, the increase in posterior precision enabled by higher effort in the private sector may offset the incentives for over-treatment under market incentives, yielding no net difference in the provision of unnecessary treatment. Overall, our results suggest that in low $\phi$ environments, the effort advantage of the private sector may outweigh the credence good costs of privately-provided healthcare.

Indian and global health policy debates have been hampered by a lack of empirical evidence on the quality of clinical interactions in the public and private sectors. Under the status quo, considerable attention has been focused on inadequate access to publicly-provided healthcare and the need to increase spending on the public healthcare system (Planning Commission of India, 2013). Our results suggest that enthusiasm for the public sector as the primary source of primary care services in resource poor settings has to be tempered by the extent to which administrative accountability is enforced in the system, and that poor incentives for effort may be a binding constraint to quality in the public system of healthcare delivery (where providers are more qualified). ${ }^{29}$

health and education appear to make up for lower qualifications with higher effort, yielding outcomes no worse than those provided by the public sector - which have much higher costs per student/patient.

${ }^{29}$ Designing incentives for public healthcare systems is a non-trivial problem, since fee-for-service compensation models are likely to induce over-treatment (Clemens and Gottlieb, 2014). On the other hand, it is also worth noting that the status quo public healthcare system in India provides negative incentives to doctors for exerting effort, since greater effort is likely to lead to an increased load of patients with no increase in compensation. One option worth evaluating could be the use of a capitation-fee based model that compensates providers for the number of patients who register with their practice (rewarding a long-term reputation for quality), but that limits financial incentives for over-treating patients in a given interaction. 
On the other hand, the marginal returns to better training and credentialing may be higher for private healthcare providers (who have better incentives for effort). However, current policy thinking often points in the opposite direction, with a focus on hiring, training, and capacity building in the public sector on one hand (without much attention to their incentives for effort), and considerable resistance to training and providing legitimacy to unqualified private providers on the other (Reddy et al., 2011; Shiva Kumar et al., 2011; Planning Commission of India, 2013).

This viewpoint is often justified by assuming that patients - particularly those who are poor and illiterate - make poor decisions regarding their health care. While certainly possible, a more nuanced understanding of patient behavior in low-income settings requires better empirical evidence on the actual quality of care obtained from different types of healthcare providers. Our paper presents some of the first evidence on this question, and expanding this methodology to other conditions and settings will allow for a richer understanding of the functioning of healthcare systems in settings with low resources and administrative capacity.

\section{References}

Arrow, Kenneth J. 1963. "Uncertainty and The Welfare Economics of Medical Care." American Economic Review, 63(5): 941-973.

Ayres, Ian, and Peter Siegelman. 1995. "Race and Gender Discrimination in Bargaining for a New Car." American Economic Review, 85(3): 304-21.

Baker, George. 1992. "Incentives Contracts and Performance Measurement." Journal of Political Economy, 100: 598-614.

Banerjee, Abhijit, Angus Deaton, and Esther Duflo. 2004. "Wealth, Health, and Health Services in Rural Rajasthan." American Economic Review, Papers and Proceedings, 94(2): 326-300.

Banerjee, Abhijit V, Esther Duflo, and Rachel Glennerster. 2008. "Putting a Band-Aid on a Corpse: Incentives for Nurses in the Public Health Care System." Journal of European Economic Association, 6(23): $487-500$.

Black, R E, S Cousens, H L Johnson, and et al. 2010. "Global, regional and national causes of child mortality in 2008: a systematic analysis." The Lancet, 375(9730): 1969-87.

Chaudhury, Nazmul, Jeffery Hammer, Michael Kremer, Karthik Muralidharan, and F. Halsey Rogers. 2006. "Missing in Action: Teacher and Health Worker Absence in Developing Countries." Journal of Economic Perspectives, 20(1): 91-116.

Clemens, Joshua, and Jeffrey Gottlieb. 2014. "Do Physicians' Financial Incentives Affect Medical Treatment and Patient Health?" American Economic Review, 104(4): 1320-49.

Coarasa, Jorge, Jishnu Das, and Elizabeth Gummerson. 2014. "Evaluating the Evidence on Public and Private Sector Quality of Care in Low and Middle Income Countries."

Cohen, Jessica, Pascaline Dupas, and Simone Schaner. 2015. "Price Subsidies, Diagnostic Tests, and Targeting of Malaria Treatment." American Economic Review, 105(2): 609-645. 
CPR. 2011. "Mapping Medical Providers in Rural India: Four Key Trends." Center For Policy Research.

Das, Jishnu, Ada Kwan, Ben Daniels, Srinath Satyanarayana, Ramnath Subbaraman, Sofi Bergkvist, Ranendra K Das, Veena Das, and Madhukar Pai. 2015. "First use of the standardized patient methodology to assess quality of Tuberculosis care." Lancet Infectious Diseases, forthcoming.

Das, Jishnu, Alaka Holla, Veena Das, Manoj Mohanan, Diana Tabak, and Brian Chan. 2012. "The Quality of Medical Care in Clinics: Evidence from a Standardized Patients Study in a Low-Income Settting." Health Affairs, 31(12): 2274-2784.

Das, Jishnu, and Jeffery Hammer. 2005. "Which Doctor? Combining Vignettes and Item Response Theory to Measure Doctor Quality." Journal of Development Economics, 78(3): 348-383.

Das, Jishnu, and Jeffery Hammer. 2007. "Money for Nothing: The Dire Straits of Medical Practice in Delhi, India." Journal of Development Economics, 83(1): 1-36.

Das, Jishnu, and Natalie Bau. 2014. "The Misallocation of Pay and Productivity in the Public Sector: Evidence from the Labor Market for Teachers." Working Paper.

DHS. 2007. "National Family Health Survey (NFHS-3) 2005-2006: Key Findings."

Dulleck, Uwe, and Rudolf Kerschbamer. 2006. "On Doctors, Mechanics, and Computer Specialists: The Economics of Credence Goods." Journal of Economic Literature, 44: 5-42.

Dulleck, Uwe, Rudolf Kerschbamer, and Matthias Sutter. 2011. "The Economics of Credence Goods: An Experiment on the Role of Liability, Verifiability, Reputation and Competition." American Economic Review, 101(3): 526-555.

Faxon, David, and Claude Lenfant. 2001. "Timing is everything - motivating patients to call 911 on onset of acute myocardial infarction." Circulation, 104(11): 1210.

Gertler, Paul J, and Christel Vermeersch. 2013. "Using Performance Incentives to Improve Medical Care Productivity and Health Outcomes." Working Paper.

Goldberg, Penelopi K. 1996. "Dealer Price Discrimination in New Car Purchases: Evidence from the Consumer Expenditure Survey." Journal of Political Economy, 104(3): 622-54.

Grepin, Karen. 2014. "The role of the private sector in delivering maternal and child health services in low-income and middle-income countries: an observational, longitudinal analysis." The Lancet, 387(S7).

Gruber, Jonathan, and Maria Owings. 1996. "Physician Financial Incentives and Cesarean Section Delivery." RAND Jounal of Economics, 27(1): 99-123.

Hanna, Rema, and Iqbal Dhaliwal. 2015. "Making a Deal with the Devil: Experiental Evidence on Bureaucratic Reform in India." Working Paper.

Holmstrom, Bengt, and Paul Milgrom. 1991. "Multi-Task Principal-Agent Problems: Incentive Contracts, Asset Ownership and Job Design." Journal of Law, Economics and Organization, 7(Special Issue): $24-52$.

Jayachandran, Seema. 2014. "Incentives to Teach Badly: After-School Tutoring in Developing Countries." Journal of Development Economics, 27(1): 99-123.

Jindal, S K, D Gupta, A N Aggarwal, and R Agarwal. 2005. "Guidelines for management of asthma at primary and secondary levels of health care in India." Indian J Chest Dis Allied Sci, 47(4): 308-343.

La Forgia, Gerrard, and Somil Nagpal. 2014. Government Sponsored Health Insurance in India: Are You Covered? The World Bank, Washington, DC. 
Leonard, Kenneth L, and Melkiory C Masatu. 2005. "The Use of Direct Clinical Observation and Vignettes for Health Services Quality Evaluation in Developing Countries." Social Science and Medicine, 61(9): 1944-1951.

Muralidharan, Karthik. 2013. "Priorities for Primary Education Policy in India's 12th Five Year Plan." India Policy Forum 2012-13, 9: 1-46.

Muralidharan, Karthik, and Venkatesh Sundararaman. 2011. "Teacher Performance Pay: Experimental Evidence from India." Journal of Political Economy, 119(1): 39-77.

Muralidharan, Karthik, and Venkatesh Sundararaman. 2015. "The Aggregate Effect of School Choice: Evidence from a Two-Stage Experiment in India." Quarterly Journal of Economics, forthcoming.

Muralidharan, Karthik, Nazmul Chudhury, Jeffrey Hammer, Michael Kremer, and F Halsey Rogers. 2011. "Is there a Doctor in the House? Absent Medical Providers in India." Working Paper.

Norris, Pauline. 2002. "Reasons why mystery shopping is a useful and justifiable research method." The Pharmaceutical Journal, 272: 746-747.

Patel, V, S Chatterji, D Chisholm, and et al. 2011. "Chronic diseases and injuries in India." The Lancet, 377(9763): 413-428.

Pauly, Mark. 1980. Doctors and Their Workshops: Economic Models of Physician Behaviors. National Bureau of Economic Research Monograph, Boston, MA.

Planning Commission of India. 2013. "Health, Nutrition and Family Welfare."

Prendergast, Canice. 2003. "The Limits of Bureaucratic Inefficiency." Journal of Political Economy, 111(5): 929-958.

Reddy, S K, Vikram Patel, Prabhat Jha, Vinod K Paul, A K Shiva Kumar, and Lalit Dandona. 2011. "Towards Achievement of Universal Health Care in India by 2020: A Call to Action." The Lancet, 377(9767): 760-768.

Rethans, Jan-Joos, Ferd Sturmans, Riet Drop, Cees van der Vlueten, and Pie Hobus. 1991. "Does Competence of General Practitioners Predict Their Performance." British Medical Journal, 303(6814): 1377-1380.

Rohde, Jon E, and Hema Viswanathan. 1995. The Rural Private Practitioner. Oxford University Press, New Delhi.

Rosenzweig, Mark R. 1995. "Why are there Returns to Schooling?" American Economic Review Papers and Proceedings, 85(2): 153-158.

Schneider, Henry S. 2012. "Agency Problems And Reputation in Expert Services: Evidence from Auto Repair." The Journal of Industrial Economics, 60(3): 406-433.

Selvaraj, S, and Anup K Karan. 2009. "Deepening Health Insecurity in India: Evidence from National Sample Surveys since 1980s." Economic and Political Weekly, 44(18): 55-60.

Shiva Kumar, A K, Lincoln C Chen, Mita Choudhury, Shiban Ganju, Vijay Mahajan, Amarjeet Sinha, and Abhijit Sen. 2011. "Financing Health Care for All: Challenges and Opportunities." The Lancet, 377(9766): 668-679.

Wagstaff, Adam. 2013. "What exactly is the public-private mix in health care?"

Wolinsky, A. 1993. "Competition in the Market for Informed Expert Services." RAND Jounal of Economics, 24(3): 380-398.

World Bank. 2003. World Development Report 2004. The World Bank, Washington, DC. 


\section{Figures and Tables}

\section{Figure 1: Optimal choice of effort and treatment with high and low $\phi$ with and without market incentives}

(A)

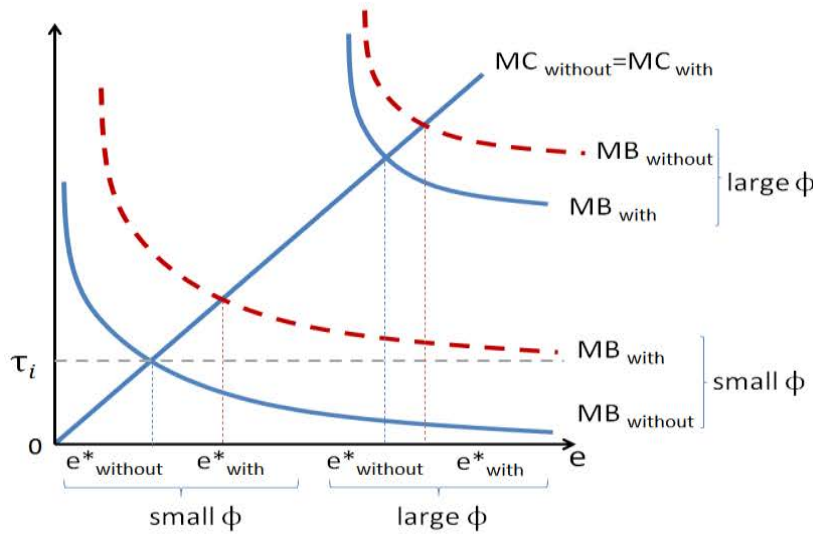

(C)

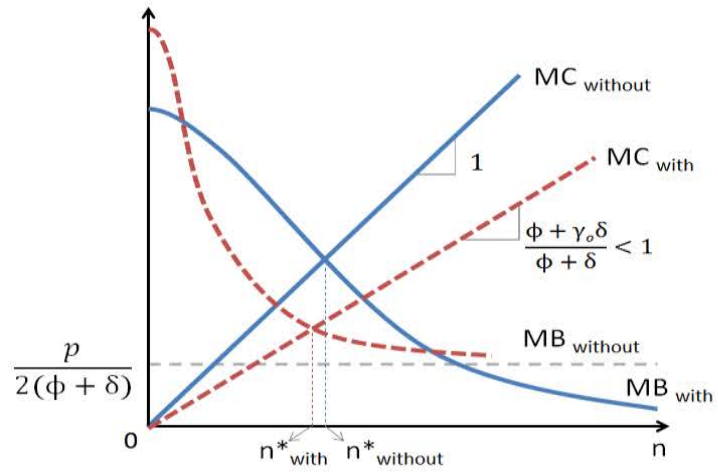

(B)

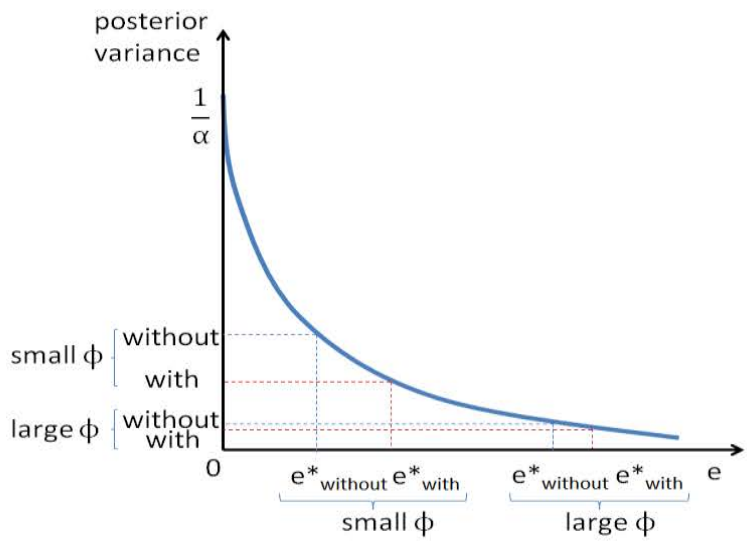

(D)

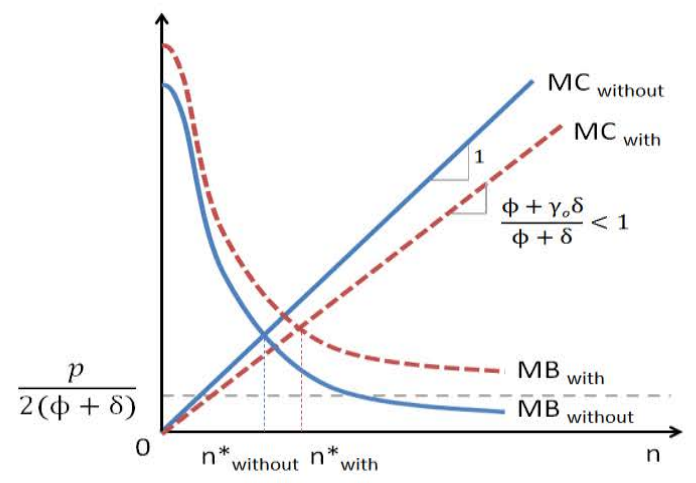

Notes: In panel (A), $\mathrm{MB}_{\text {with }}$ and $\mathrm{MC}_{\mathrm{with}}$ are the marginal benefit and the cost of $e$ with market incentives, and $\mathrm{MB}_{\text {without }}$ and $\mathrm{MC}_{\text {without }}$ are those without market incentives. $e_{\text {with }}^{*}$ and $e_{\text {without }}^{*}$ are optimal levels of effort with and without market incentives for small and large $\phi$ values. In panel (B), The graph traces the posterior variance $\frac{1}{\alpha+\beta}$ with $e$ on the $\mathrm{x}$-axis. The $\mathrm{y}$-axis intercept $\frac{1}{\alpha}$ is the posterior variance when $e=0$. In panel (C) and panel (D), $\mathrm{MB}_{\text {with }}$ and $\mathrm{MC}_{\text {with }}$ are the marginal benefit and the cost of $n$ with market incentives, and $\mathrm{MB}_{\text {without }}$ and $\mathrm{MC}_{\text {without }}$ are those without market incentives. $n_{\mathrm{with}}^{*}$ and $n_{\mathrm{without}}^{*}$ are optimal levels of treatment with and without market incentives for small and large $\phi$ values. Panel (C) and panel (D) compares the optimal level of treatment with and without market incentives when the posterior variance with market incentives is substantially smaller than that without market incentives and when the two posterior beliefs are similar.

\section{Figure 2: Health outcome with and without market incentives with varying $\phi$}

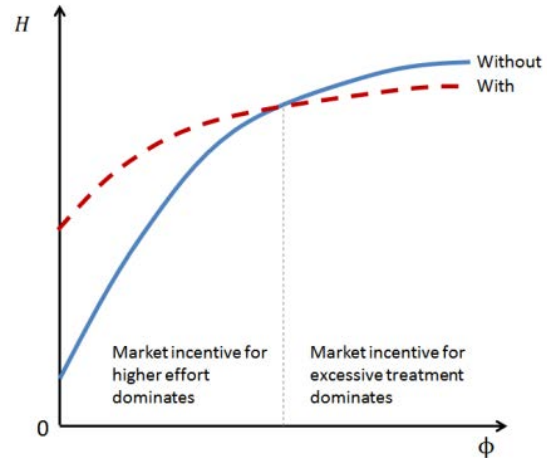

Notes: The graph illustrates the health outcome produced with and without market incentives with different values of $\phi$. The y-axis is the health outcome $H$ and $\mathrm{x}$-axis is the magnitude of low-powered incentive, $\phi$. The solid line traces $H$ without market incentives and the dotted line traces $H$ with market incentives. 


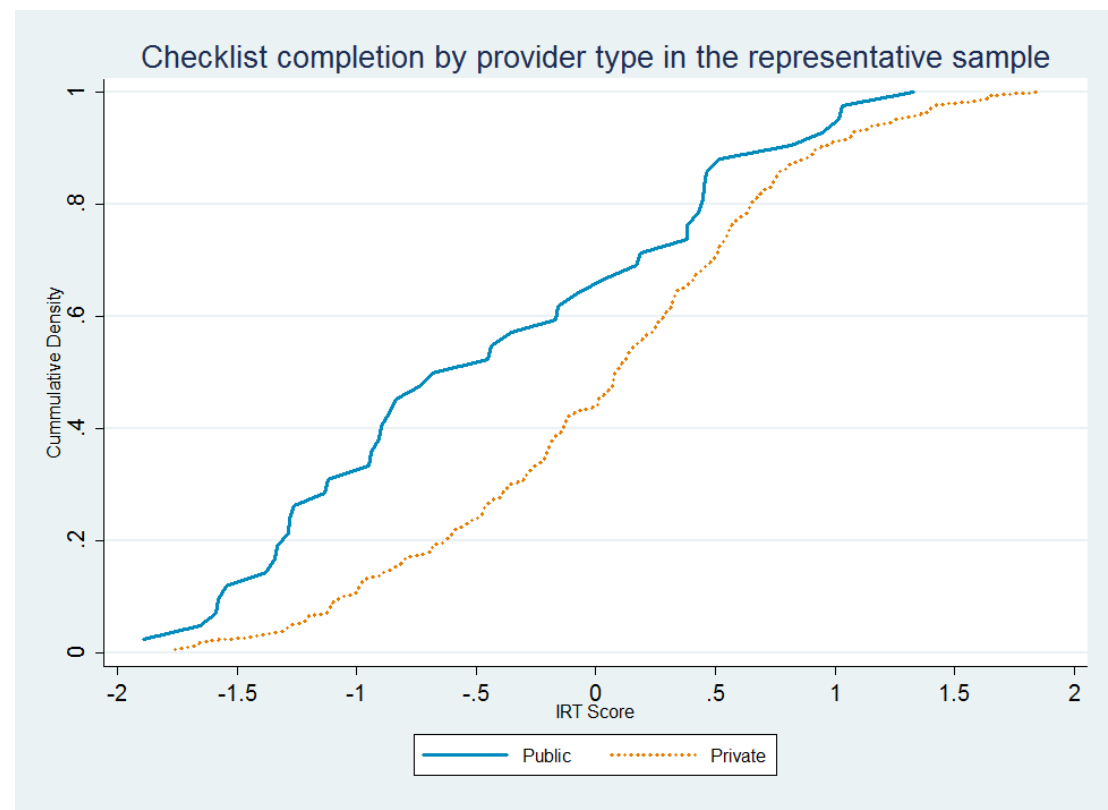

Figure 3

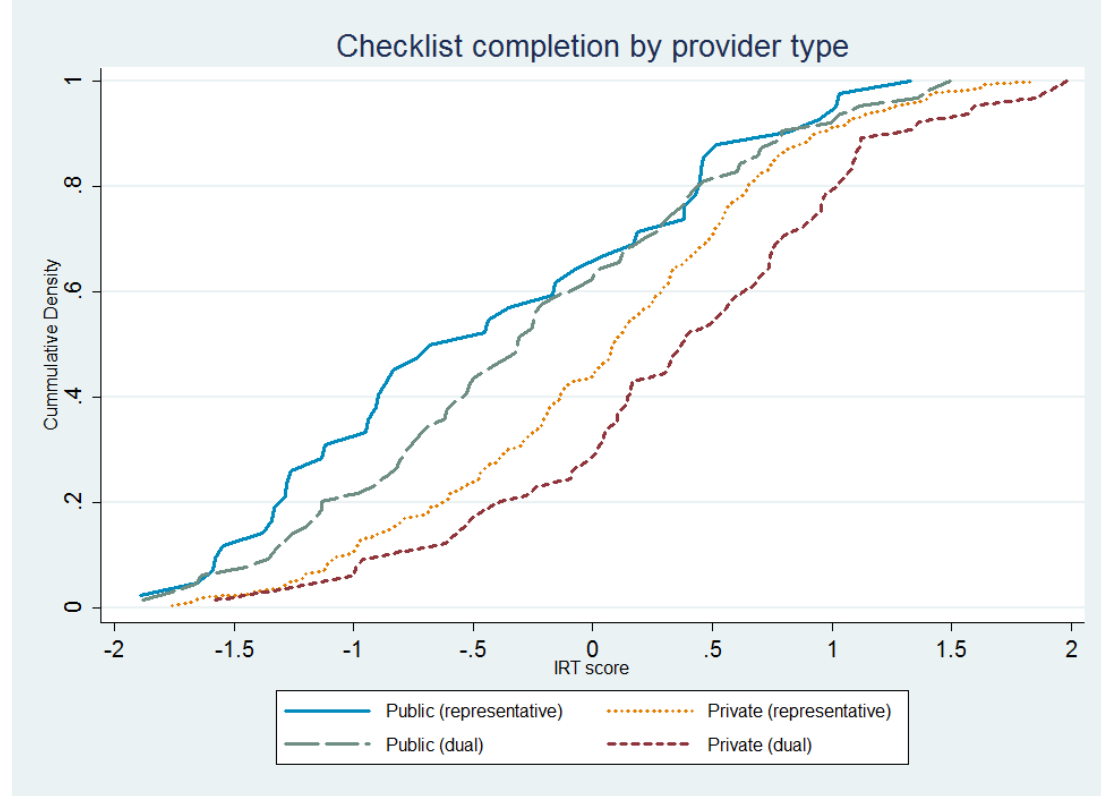

Figure 5

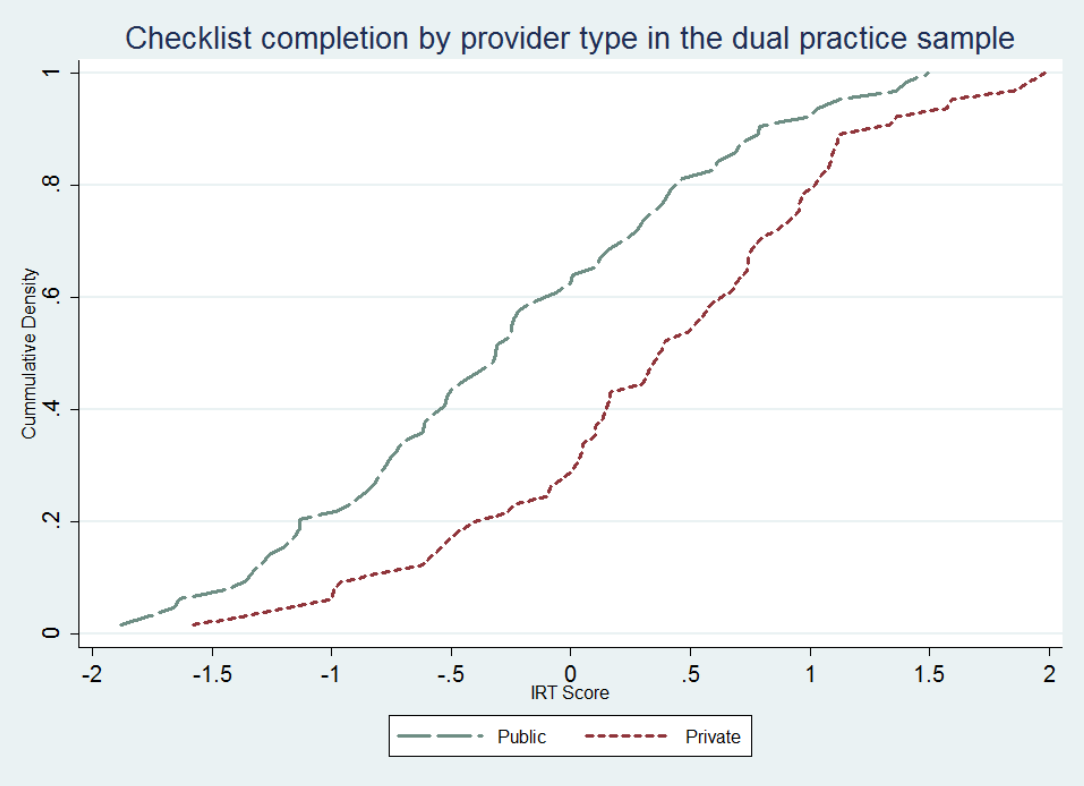

Figure 4

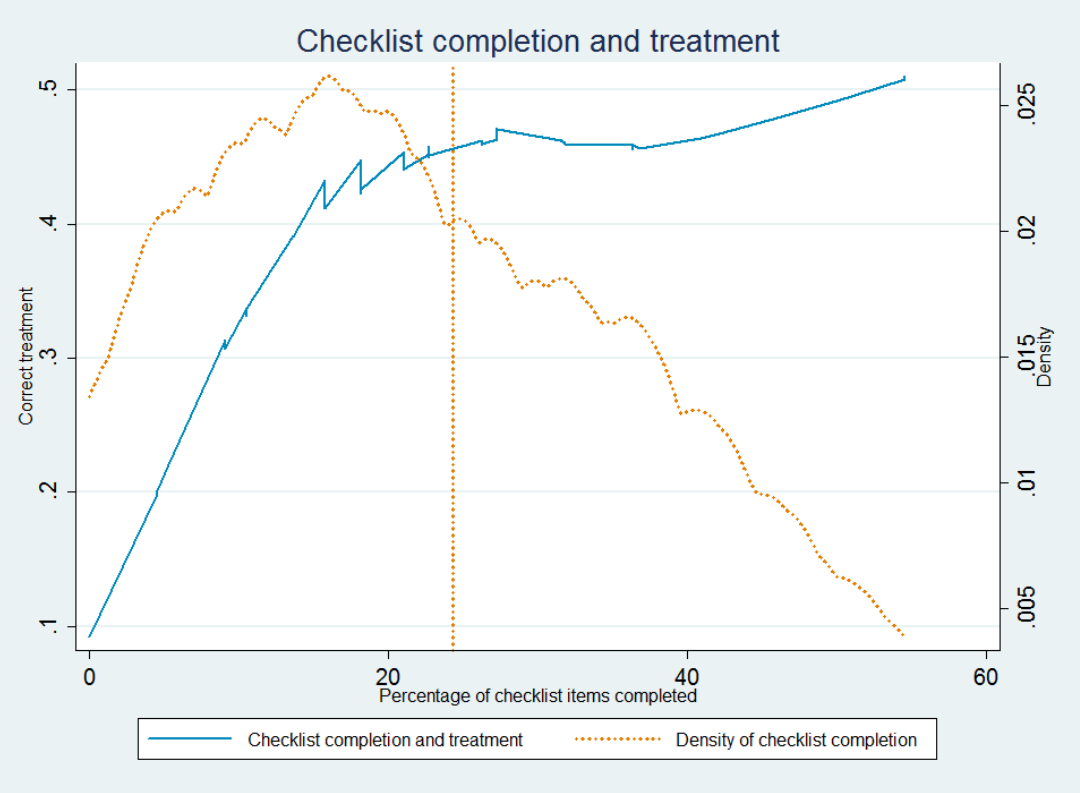

Figure 6 
Table 1: Health market attributes

\begin{tabular}{|c|c|c|c|c|c|c|}
\hline & (1) & (2) & (3) & (4) & (5) & (6) \\
\hline & \multicolumn{3}{|c|}{$\begin{array}{c}\text { Madhya Pradesh } \\
\text { (5 districts, } 100 \text { markets) }\end{array}$} & \multicolumn{3}{|c|}{$\begin{array}{l}\text { SP Sample Villages } \\
\text { (3 districts, } 46 \text { markets) }\end{array}$} \\
\hline & All & $\begin{array}{l}\text { Inside } \\
\text { village }\end{array}$ & $\begin{array}{l}\text { Outside } \\
\text { village }\end{array}$ & All & $\begin{array}{l}\text { Inside } \\
\text { village }\end{array}$ & $\begin{array}{l}\text { Outside } \\
\text { village }\end{array}$ \\
\hline \multicolumn{7}{|c|}{ Panel A: Composition of markets based on census of providers } \\
\hline Total & $\begin{array}{c}11.68 \\
(12.06)\end{array}$ & $\begin{array}{c}3.97 \\
(4.49)\end{array}$ & $\begin{array}{c}7.71 \\
(12.17)\end{array}$ & $\begin{array}{c}16.02 \\
(15.81)\end{array}$ & $\begin{array}{c}4.65 \\
(5.41)\end{array}$ & $\begin{array}{l}11.37 \\
(16.42)\end{array}$ \\
\hline Public MBBS & $\begin{array}{c}0.45 \\
(0.97)\end{array}$ & $\begin{array}{c}0.05 \\
(0.22)\end{array}$ & $\begin{array}{c}0.40 \\
(0.93)\end{array}$ & $\begin{array}{c}0.50 \\
(1.11)\end{array}$ & $\begin{array}{c}0.02 \\
(0.15)\end{array}$ & $\begin{array}{c}0.48 \\
(1.11)\end{array}$ \\
\hline Public alternative qualification & $\begin{array}{c}0.22 \\
(0.48)\end{array}$ & $\begin{array}{c}0.07 \\
(0.29)\end{array}$ & $\begin{array}{c}0.15 \\
(0.39)\end{array}$ & $\begin{array}{c}0.24 \\
(0.52)\end{array}$ & $\begin{array}{c}0.07 \\
(0.33)\end{array}$ & $\begin{array}{c}0.17 \\
(0.44)\end{array}$ \\
\hline Public paramedical & $\begin{array}{c}1.58 \\
(1.90)\end{array}$ & $\begin{array}{c}1.13 \\
(1.46)\end{array}$ & $\begin{array}{c}0.45 \\
(1.33)\end{array}$ & $\begin{array}{c}1.98 \\
(2.12)\end{array}$ & $\begin{array}{c}1.30 \\
(1.49)\end{array}$ & $\begin{array}{l}0.67 \\
(1.59)\end{array}$ \\
\hline Public unqualified & $\begin{array}{c}1.71 \\
(1.75)\end{array}$ & $\begin{array}{c}0.68 \\
(1.04)\end{array}$ & $\begin{array}{l}1.03 \\
(1.54)\end{array}$ & $\begin{array}{c}2.07 \\
(2.05)\end{array}$ & $\begin{array}{c}0.67 \\
(1.12)\end{array}$ & $\begin{array}{c}1.39 \\
(1.94)\end{array}$ \\
\hline Total public & $\begin{array}{l}3.96 \\
(3.20)\end{array}$ & $\begin{array}{c}1.93 \\
(2.28)\end{array}$ & $\begin{array}{c}2.03 \\
(2.63)\end{array}$ & $\begin{array}{c}4.78 \\
(3.53)\end{array}$ & $\begin{array}{c}2.07 \\
(2.45)\end{array}$ & $\begin{array}{c}2.72 \\
(3.17)\end{array}$ \\
\hline Private MBBS & $\begin{array}{c}0.40 \\
(1.57)\end{array}$ & $\begin{array}{c}0.00 \\
(0.00)\end{array}$ & $\begin{array}{c}0.40 \\
(1.57)\end{array}$ & $\begin{array}{c}0.59 \\
(2.15)\end{array}$ & $\begin{array}{c}0.00 \\
(0.00)\end{array}$ & $\begin{array}{c}0.59 \\
(2.15)\end{array}$ \\
\hline Private alternative qualification & $\begin{array}{c}1.92 \\
(3.65)\end{array}$ & $\begin{array}{c}0.23 \\
(0.66)\end{array}$ & $\begin{array}{c}1.69 \\
(3.65)\end{array}$ & $\begin{array}{c}2.67 \\
(4.86)\end{array}$ & $\begin{array}{c}0.33 \\
(0.90)\end{array}$ & $\begin{array}{c}2.35 \\
(4.89)\end{array}$ \\
\hline Private unqualified & $\begin{array}{c}5.40 \\
(6.01)\end{array}$ & $\begin{array}{c}1.81 \\
(2.23)\end{array}$ & $\begin{array}{c}3.59 \\
(6.14)\end{array}$ & $\begin{array}{c}7.98 \\
(7.88)\end{array}$ & $\begin{array}{c}2.26 \\
(2.74)\end{array}$ & $\begin{array}{c}5.72 \\
(8.32)\end{array}$ \\
\hline Total private & $\begin{array}{c}7.72 \\
(10.54) \\
\end{array}$ & $\begin{array}{c}2.04 \\
(2.69) \\
\end{array}$ & $\begin{array}{c}5.68 \\
(10.81) \\
\end{array}$ & $\begin{array}{c}11.24 \\
(14.31) \\
\end{array}$ & $\begin{array}{r}2.59 \\
(3.38) \\
\end{array}$ & $\begin{array}{c}8.65 \\
(14.87) \\
\end{array}$ \\
\hline \multicolumn{7}{|c|}{ Panel B: Composition of demand from census of households in sampled villages } \\
\hline $\begin{array}{l}\text { Fraction of households that visited a } \\
\text { provider in last } 30 \text { days }\end{array}$ & $\begin{array}{c}0.46 \\
(0.50)\end{array}$ & & & $\begin{array}{c}0.58 \\
(0.49)\end{array}$ & & \\
\hline Fraction provider visits inside/outside village & & $\begin{array}{c}0.66 \\
(0.47)\end{array}$ & $\begin{array}{c}0.34 \\
(0.47)\end{array}$ & & $\begin{array}{c}0.69 \\
(0.46)\end{array}$ & $\begin{array}{c}0.31 \\
(0.46)\end{array}$ \\
\hline Distance traveled to visited provider $(\mathrm{km})$ & $\begin{array}{c}1.61 \\
(2.14)\end{array}$ & $\begin{array}{c}0.40 \\
(0.65)\end{array}$ & $\begin{array}{c}3.83 \\
(2.14)\end{array}$ & $\begin{array}{c}1.37 \\
(2.37)\end{array}$ & $\begin{array}{c}0.38 \\
(1.16)\end{array}$ & $\begin{array}{c}3.51 \\
(2.84)\end{array}$ \\
\hline Fraction of visits to MBBS doctor & $\begin{array}{c}0.04 \\
(0.19)\end{array}$ & $\begin{array}{c}0.01 \\
(0.09)\end{array}$ & $\begin{array}{c}0.09 \\
(0.29)\end{array}$ & $\begin{array}{c}0.02 \\
(0.13)\end{array}$ & $\begin{array}{c}0.00 \\
(0.00)\end{array}$ & $\begin{array}{c}0.06 \\
(0.23)\end{array}$ \\
\hline Fraction of visits to private sector & $\begin{array}{c}0.89 \\
(0.31)\end{array}$ & $\begin{array}{c}0.92 \\
(0.28)\end{array}$ & $\begin{array}{c}0.85 \\
(0.36)\end{array}$ & $\begin{array}{c}0.96 \\
(0.21)\end{array}$ & $\begin{array}{c}0.97 \\
(0.18)\end{array}$ & $\begin{array}{c}0.93 \\
(0.26)\end{array}$ \\
\hline $\begin{array}{l}\text { Fraction of visits to private sector } \\
\text { (conditional on public availability) }\end{array}$ & $\begin{array}{c}0.88 \\
(0.33)\end{array}$ & $\begin{array}{c}0.89 \\
(0.31)\end{array}$ & $\begin{array}{c}0.83 \\
(0.38)\end{array}$ & $\begin{array}{c}0.95 \\
(0.22)\end{array}$ & $\begin{array}{c}0.96 \\
(0.20)\end{array}$ & $\begin{array}{c}0.91 \\
(0.28)\end{array}$ \\
\hline $\begin{array}{l}\text { Fraction of visits to private sector } \\
\text { (conditional on public MBBS availability) }\end{array}$ & $\begin{array}{c}0.83 \\
(0.37)\end{array}$ & $\begin{array}{c}0.84 \\
(0.36)\end{array}$ & $\begin{array}{c}0.79 \\
(0.41)\end{array}$ & $\begin{array}{c}0.93 \\
(0.25)\end{array}$ & $\begin{array}{c}0.98 \\
(0.15)\end{array}$ & $\begin{array}{c}0.90 \\
(0.30)\end{array}$ \\
\hline Fraction of visits to unqualified providers & $\begin{array}{c}0.77 \\
(0.42)\end{array}$ & $\begin{array}{c}0.87 \\
(0.34)\end{array}$ & $\begin{array}{c}0.55 \\
(0.50)\end{array}$ & $\begin{array}{c}0.82 \\
(0.39)\end{array}$ & $\begin{array}{c}0.89 \\
(0.31)\end{array}$ & $\begin{array}{c}0.64 \\
(0.48)\end{array}$ \\
\hline $\begin{array}{l}\text { Fraction of visits to unqualified providers } \\
\text { (conditional on public availability) }\end{array}$ & $\begin{array}{c}0.74 \\
(0.44)\end{array}$ & $\begin{array}{c}0.82 \\
(0.38)\end{array}$ & $\begin{array}{c}0.54 \\
(0.50)\end{array}$ & $\begin{array}{c}0.81 \\
(0.39)\end{array}$ & $\begin{array}{c}0.86 \\
(0.35)\end{array}$ & $\begin{array}{c}0.64 \\
(0.48)\end{array}$ \\
\hline $\begin{array}{l}\text { Fraction of visits to unqualified providers } \\
\text { (conditional on public MBBS availability) }\end{array}$ & $\begin{array}{c}0.60 \\
(0.49) \\
\end{array}$ & $\begin{array}{c}0.77 \\
(0.42) \\
\end{array}$ & $\begin{array}{c}0.38 \\
(0.48) \\
\end{array}$ & $\begin{array}{c}0.66 \\
(0.47)\end{array}$ & $\begin{array}{c}0.81 \\
(0.39)\end{array}$ & $\begin{array}{r}0.39 \\
(0.49) \\
\end{array}$ \\
\hline \multicolumn{7}{|c|}{ Panel C: Sample Characteristics from household census of provider choice } \\
\hline Number of villages & 100 & & & 46 & & \\
\hline Average village population & 1,149 & & & 1,199 & & \\
\hline Average number of households per village & 233 & & & 239 & & \\
\hline Number of reported provider visits & 19,331 & & & 12,122 & & \\
\hline Average number of visits per household per & 0.83 & & & 1.10 & & \\
\hline
\end{tabular}


Table 2: Characteristics of providers and practices where SPs were administered
(1)
(2)
(3)
(4)
(5)
(6)
(7)

(8)

(9)

(10)

Representative sample

Representative sample of Public MBBS

(3 districts)

providers

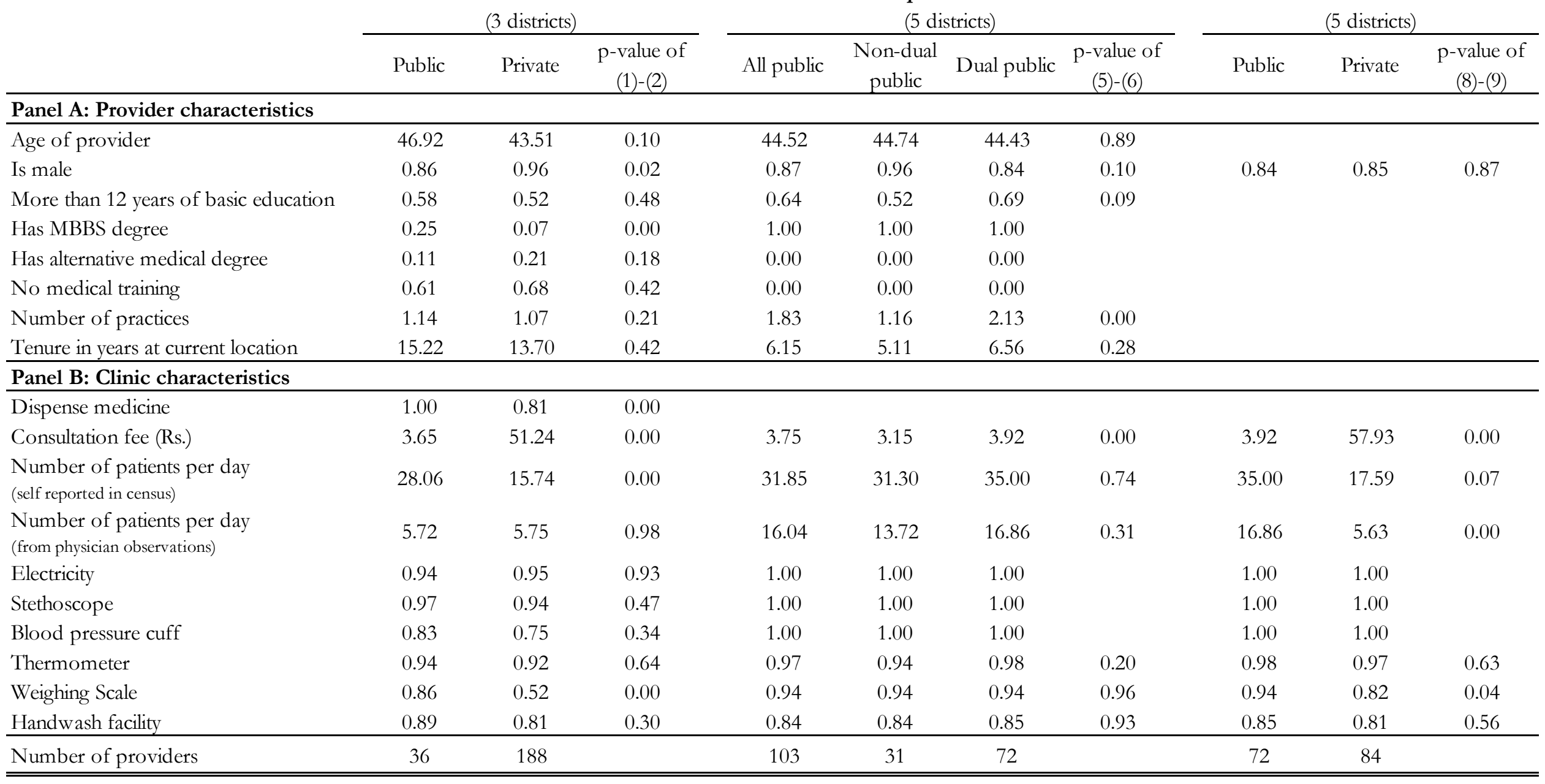

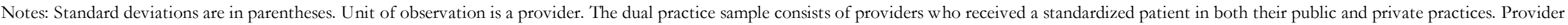

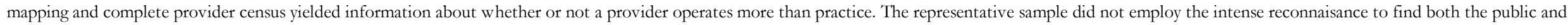

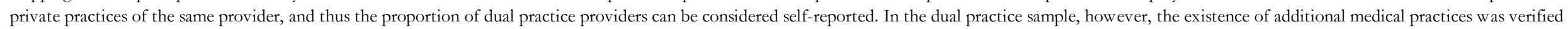

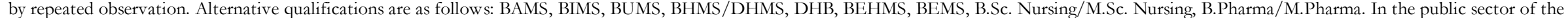
representative sample, there are 3 providers with BAMS and 1 with B.Pharma/M.Pharma. In the private sector, there are 21 with BAMS, 9 with BHMS/DHMS, 3 each with BIMS and DHB, 2 with

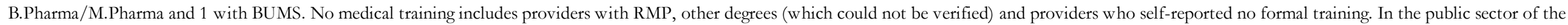

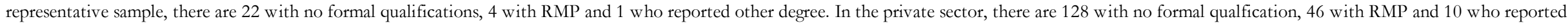
other degrees. Means for consultation fee were calculated from direct observations of clinical interactions. All other variables derive from a survey administered during the census of providers. 
Table 3: Effort in the public and private sectors

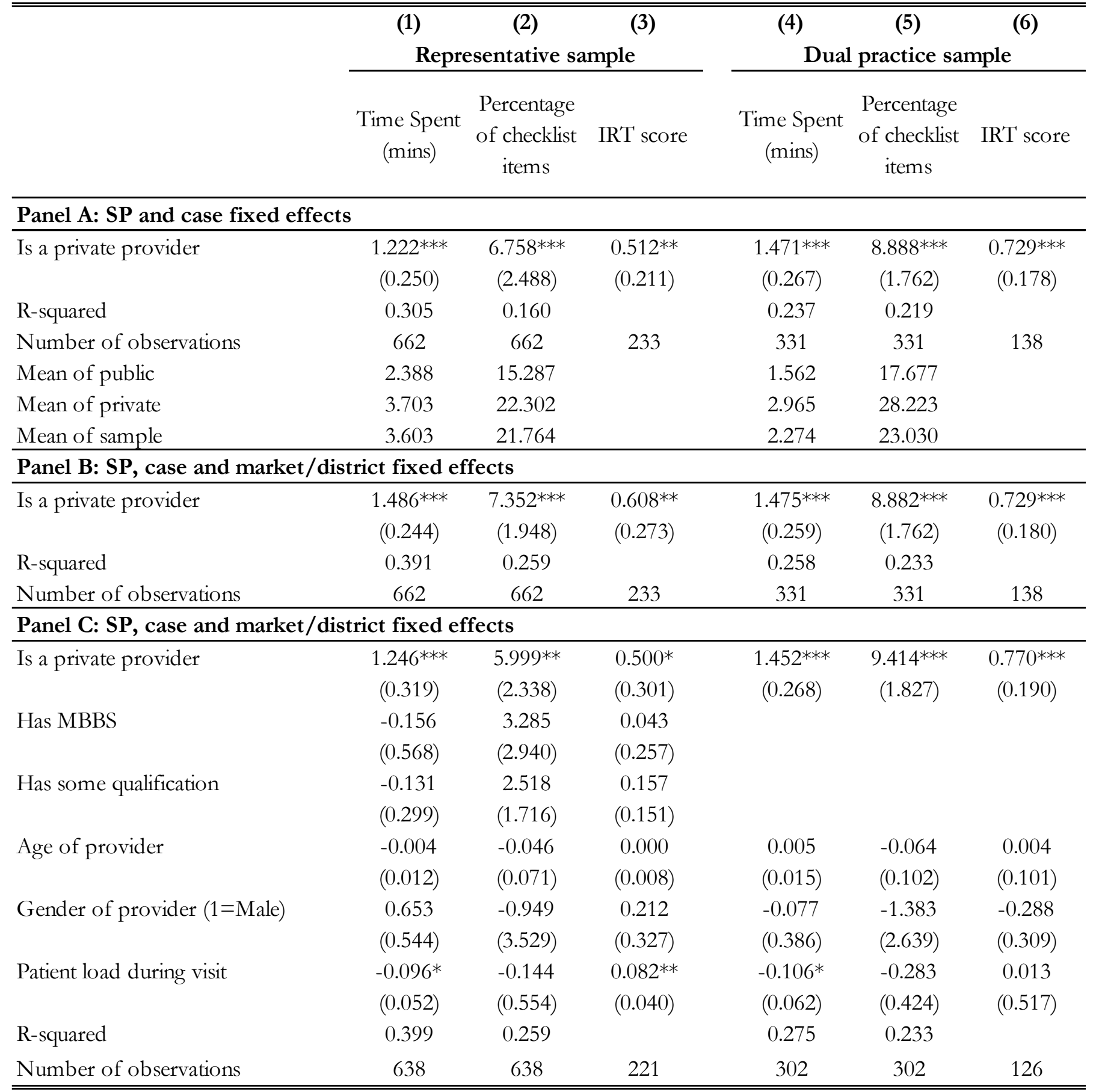

Notes: *** Significant at $1 \%$, ** Significant at $5 \%, *$ Significant at $10 \%$. Robust standard errors clustered at the market level are in parenthesis. All regressions include a constant. Observations are standardized provider-patient interactions, except in IRT score column where each observation is a provider. The score is computed using all cases, plausible values scores are used. Market fixed effects are used for the representative sample, and district fixed effects for dual practice sample. 
Table 4: Diagnosis in the public and private sectors (unstable angina and asthma cases only)

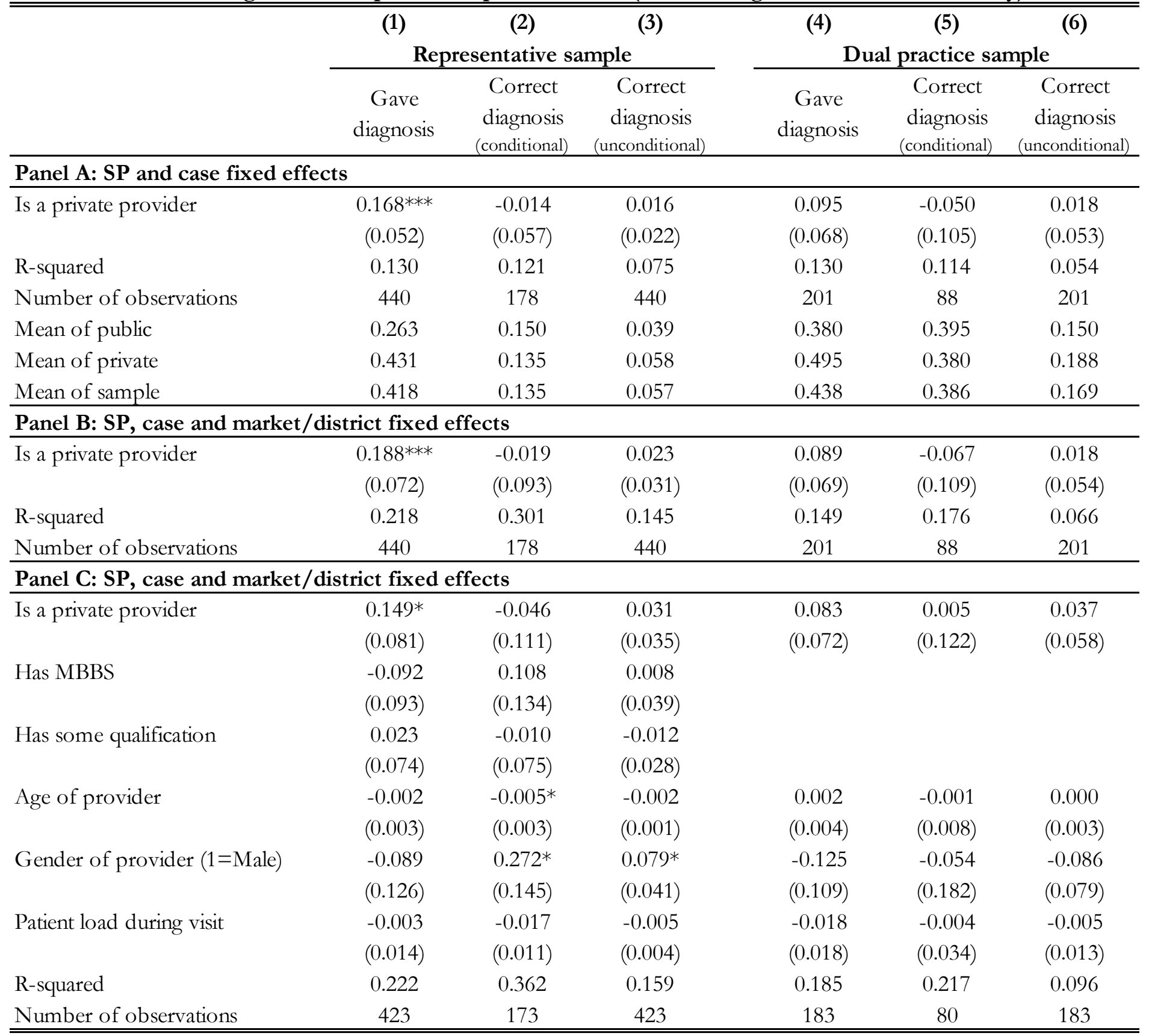

Notes: *** Significant at $1 \%, * *$ Significant at $5 \%, *$ Significant at $10 \%$. Robust standard errors clustered at the market level are in parenthesis. All regressions include a constant. Observations are standardized provider-patient interactions. Market fixed effects are used for the representative sample, and district fixed effects for dual practice sample. 
Table 5: Treatment in the public and private sectors (unstable angina and asthma cases only)

\begin{tabular}{|c|c|c|c|c|c|c|c|c|c|c|c|c|}
\hline & (1) & (2) & (3) & (4) & (5) & (6) & (7) & (8) & (9) & (10) & (11) & (12) \\
\hline & \multicolumn{6}{|c|}{ Representative sample } & \multicolumn{6}{|c|}{ Dual practice sample } \\
\hline & $\begin{array}{l}\text { Correct } \\
\text { treatment }\end{array}$ & $\begin{array}{l}\text { Palliative } \\
\text { treatment }\end{array}$ & $\begin{array}{c}\text { Unnecessary } \\
\text { treatment }\end{array}$ & $\begin{array}{c}\text { Correct } \\
\text { treatment } \\
\text { only }\end{array}$ & Antibiotic & $\begin{array}{c}\text { Number of } \\
\text { medicines }\end{array}$ & $\begin{array}{l}\text { Correct } \\
\text { treatment }\end{array}$ & $\begin{array}{l}\text { Palliative } \\
\text { treatment }\end{array}$ & $\begin{array}{c}\text { Unnecessary } \\
\text { treatment }\end{array}$ & $\begin{array}{c}\text { Correct } \\
\text { treatment } \\
\text { only } \\
\end{array}$ & Antibiotic & $\begin{array}{c}\text { Number of } \\
\text { medicines }\end{array}$ \\
\hline \multicolumn{13}{|c|}{ Panel A: SP and case fixed effects } \\
\hline \multirow[t]{2}{*}{ Is a private provider } & 0.052 & -0.038 & 0.061 & -0.008 & 0.016 & $0.972 * * *$ & $0.138^{* *}$ & $-0.113^{*}$ & -0.014 & 0.018 & $-0.119 *$ & 0.064 \\
\hline & $(0.045)$ & $(0.056)$ & $(0.072)$ & $(0.023)$ & $(0.062)$ & $(0.279)$ & $(0.064)$ & $(0.061)$ & $(0.051)$ & $(0.025)$ & $(0.068)$ & $(0.182)$ \\
\hline R-squared & 0.260 & 0.215 & 0.066 & 0.044 & 0.079 & 0.087 & 0.270 & 0.306 & 0.107 & 0.025 & 0.114 & 0.128 \\
\hline Number of observations & 440 & 440 & 440 & 440 & 440 & 440 & 201 & 201 & 201 & 201 & 201 & 201 \\
\hline Mean of public & 0.211 & 0.526 & 0.737 & 0.026 & 0.263 & 2.092 & 0.380 & 0.630 & 0.830 & 0.020 & 0.480 & 2.800 \\
\hline Mean of private & 0.270 & 0.496 & 0.808 & 0.017 & 0.279 & 3.097 & 0.554 & 0.475 & 0.842 & 0.040 & 0.386 & 2.950 \\
\hline Mean of sample & 0.266 & 0.498 & 0.802 & 0.018 & 0.278 & 3.021 & 0.468 & 0.552 & 0.836 & 0.030 & 0.433 & 2.876 \\
\hline \multicolumn{13}{|c|}{ Panel B: SP, case and market/district fixed effects } \\
\hline \multirow[t]{2}{*}{ Is a private provider } & 0.051 & 0.040 & 0.095 & -0.020 & 0.086 & $0.894 * * *$ & $0.140^{* *}$ & $-0.116^{*}$ & -0.014 & 0.017 & $-0.121 *$ & 0.052 \\
\hline & $(0.059)$ & $(0.068)$ & $(0.070)$ & $(0.026)$ & $(0.069)$ & $(0.234)$ & $(0.064)$ & $(0.061)$ & $(0.050)$ & $(0.026)$ & $(0.068)$ & $(0.181)$ \\
\hline R-squared & 0.384 & 0.350 & 0.233 & 0.255 & 0.239 & 0.289 & 0.294 & 0.312 & 0.166 & 0.039 & 0.130 & 0.155 \\
\hline Number of observations & 440 & 440 & 440 & 440 & 440 & 440 & 201 & 201 & 201 & 201 & 201 & 201 \\
\hline \multicolumn{13}{|c|}{ Panel C: SP, case and market/district fixed effects } \\
\hline \multirow[t]{2}{*}{ Is a private provider } & 0.101 & 0.060 & 0.066 & -0.005 & 0.112 & $0.638^{* *}$ & $0.160^{* *}$ & -0.095 & -0.014 & 0.017 & -0.100 & 0.045 \\
\hline & $(0.071)$ & $(0.080)$ & $(0.075)$ & $(0.027)$ & $(0.080)$ & $(0.284)$ & $(0.068)$ & $(0.064)$ & $(0.057)$ & $(0.028)$ & $(0.071)$ & $(0.192)$ \\
\hline \multirow[t]{2}{*}{ Has MBBS } & $0.309^{* * *}$ & $0.246^{* *}$ & -0.132 & $0.106^{* *}$ & $0.267 * * *$ & -0.397 & & & & & & \\
\hline & $(0.087)$ & $(0.100)$ & $(0.089)$ & $(0.051)$ & $(0.086)$ & $(0.352)$ & & & & & & \\
\hline \multirow[t]{2}{*}{ Has some qualification } & 0.088 & 0.086 & 0.029 & -0.001 & 0.099 & -0.116 & & & & & & \\
\hline & $(0.057)$ & $(0.066)$ & $(0.054)$ & $(0.014)$ & $(0.063)$ & $(0.241)$ & & & & & & \\
\hline \multirow[t]{2}{*}{ Age of provider } & -0.001 & -0.001 & -0.002 & -0.000 & -0.000 & -0.012 & -0.002 & $-0.007^{*}$ & 0.001 & -0.002 & -0.001 & $-0.019 *$ \\
\hline & $(0.002)$ & $(0.003)$ & $(0.002)$ & $(0.000)$ & $(0.003)$ & $(0.010)$ & $(0.004)$ & $(0.004)$ & $(0.003)$ & $(0.001)$ & $(0.004)$ & $(0.011)$ \\
\hline \multirow[t]{2}{*}{ Gender of provider ( $1=$ Male $)$} & 0.133 & -0.118 & -0.068 & 0.001 & -0.029 & -0.128 & 0.049 & 0.097 & 0.111 & 0.007 & 0.152 & 0.285 \\
\hline & $(0.098)$ & $(0.122)$ & $(0.091)$ & $(0.033)$ & $(0.132)$ & $(0.332)$ & $(0.100)$ & $(0.090)$ & $(0.081)$ & $(0.038)$ & $(0.100)$ & $(0.290)$ \\
\hline \multirow[t]{2}{*}{ Patient load during visit } & -0.008 & -0.017 & 0.007 & -0.001 & -0.008 & 0.009 & 0.001 & 0.005 & 0.014 & -0.004 & 0.002 & $0.076^{*}$ \\
\hline & $(0.010)$ & $(0.011)$ & $(0.008)$ & $(0.001)$ & $(0.008)$ & $(0.045)$ & $(0.015)$ & $(0.014)$ & $(0.017)$ & $(0.003)$ & $(0.016)$ & $(0.040)$ \\
\hline R-squared & 0.406 & 0.370 & 0.253 & 0.278 & 0.272 & 0.293 & 0.273 & 0.316 & 0.180 & 0.053 & 0.159 & 0.180 \\
\hline Number of observations & 423 & 423 & 423 & 423 & 423 & 423 & 183 & 183 & 183 & 183 & 183 & 183 \\
\hline
\end{tabular}

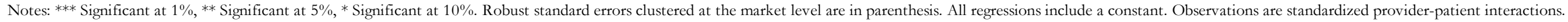

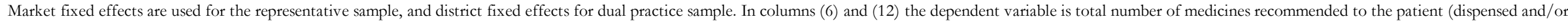
prescribed). 
Table 6: Correlates of price charged (private interactions)

\begin{tabular}{|c|c|c|c|c|c|c|}
\hline & (1) & (2) & (3) & (4) & (5) & (6) \\
\hline & \multirow{2}{*}{\multicolumn{6}{|c|}{$\begin{array}{c}\text { Fees in Rs. } \\
\text { Dual practice sample }\end{array}$}} \\
\hline & \multicolumn{2}{|c|}{ Representative sample } & & Dual practice sample & Pooled sample & \\
\hline & $\begin{array}{c}\text { Binary } \\
\text { regressions }\end{array}$ & $\begin{array}{c}\text { Multiple } \\
\text { regression }\end{array}$ & $\begin{array}{c}\text { Binary } \\
\text { regressions }\end{array}$ & $\begin{array}{l}\text { Multiple } \\
\text { regression }\end{array}$ & $\begin{array}{c}\text { Binary } \\
\text { regressions }\end{array}$ & $\begin{array}{l}\text { Multiple } \\
\text { regression }\end{array}$ \\
\hline \multirow[t]{2}{*}{ Time spent with SP (minutes) } & $1.763^{* * *}$ & 0.771 & $2.625^{* * *}$ & $2.138^{* * *}$ & $1.530 * * *$ & $0.839 * *$ \\
\hline & $(0.454)$ & $(0.475)$ & $(0.587)$ & $(0.678)$ & $(0.363)$ & $(0.393)$ \\
\hline \multirow[t]{2}{*}{ Percentage of checklist items } & $0.411^{* * *}$ & $0.368^{* * *}$ & $0.364^{* * *}$ & 0.063 & $0.396^{* * *}$ & $0.312^{* * *}$ \\
\hline & $(0.091)$ & $(0.101)$ & $(0.100)$ & $(0.125)$ & $(0.073)$ & $(0.092)$ \\
\hline \multirow[t]{2}{*}{ Correct diagnosis (unconditional) } & -3.749 & -2.137 & 7.504 & 6.022 & 3.026 & 3.051 \\
\hline & $(4.212)$ & $(2.122)$ & $(9.350)$ & $(9.080)$ & $(4.678)$ & $(4.192)$ \\
\hline \multirow[t]{2}{*}{ Correct treatment } & $7.065^{* * *}$ & 0.050 & 7.744* & 3.800 & $7.978^{* * *}$ & 1.969 \\
\hline & $(1.789)$ & $(2.892)$ & $(4.145)$ & $(4.955)$ & $(1.895)$ & $(2.351)$ \\
\hline \multirow[t]{2}{*}{ Palliative treatment } & $8.036^{* * *}$ & $5.581 * * *$ & $10.435^{* *}$ & 7.381 & $7.890^{* * *}$ & $6.125^{* * *}$ \\
\hline & $(2.056)$ & $(2.036)$ & $(4.242)$ & $(4.602)$ & $(1.807)$ & $(1.863)$ \\
\hline \multirow[t]{2}{*}{ Unnecessary treatment } & $14.039 * * *$ & 4.030 & $14.973^{* * *}$ & 4.263 & $14.283^{* * *}$ & $5.681 * *$ \\
\hline & $(2.395)$ & $(3.341)$ & $(5.032)$ & $(6.181)$ & $(2.126)$ & $(2.855)$ \\
\hline \multirow[t]{2}{*}{ Number of medicines dispensed } & $4.774 * * *$ & $4.215^{* * *}$ & $9.517 * * *$ & $10.675^{* * *}$ & $4.101 * * *$ & $3.840 * * *$ \\
\hline & $(1.656)$ & $(1.379)$ & $(3.035)$ & (3.785) & $(1.373)$ & $(1.402)$ \\
\hline \multirow[t]{2}{*}{ Number of medicines prescribed } & -0.202 & -1.188 & $3.169 *$ & 2.895 & 0.834 & -1.213 \\
\hline & $(1.129)$ & $(0.881)$ & $(1.885)$ & $(2.780)$ & $(0.863)$ & $(1.071)$ \\
\hline \multirow[t]{2}{*}{ Referred/Asked to see child } & $-19.161 * * *$ & $-13.301 * * *$ & $-9.882 * *$ & -4.554 & $-16.737 * * *$ & $-14.223^{* * *}$ \\
\hline & $(4.115)$ & $(3.636)$ & $(4.763)$ & $(4.601)$ & $(3.366)$ & (3.205) \\
\hline \multirow[t]{2}{*}{ Has MBBS } & $24.325^{* * *}$ & $28.416 * * *$ & & & $14.504 * * *$ & $22.130 * * *$ \\
\hline & $(6.644)$ & (7.997) & & & $(4.608)$ & $(4.205)$ \\
\hline \multirow[t]{2}{*}{ Has some qualification } & 4.444 & $5.399 * *$ & & & 2.317 & $6.087 * * *$ \\
\hline & $(3.276)$ & $(2.139)$ & & & $(2.930)$ & $(2.185)$ \\
\hline \multirow[t]{2}{*}{ Patient load during visit } & 0.736 & 0.441 & -0.073 & -0.311 & 0.402 & 0.068 \\
\hline & $(0.665)$ & $(0.333)$ & $(0.807)$ & $(0.812)$ & $(0.621)$ & $(0.522)$ \\
\hline \multirow[t]{2}{*}{ Age of provider } & -0.150 & -0.103 & 0.267 & 0.254 & -0.091 & -0.013 \\
\hline & $(0.144)$ & $(0.091)$ & $(0.239)$ & $(0.218)$ & $(0.119)$ & $(0.083)$ \\
\hline \multirow[t]{2}{*}{ Gender of provider (1=Male) } & $-8.164 * *$ & -4.923 & -1.284 & -4.168 & $-7.555^{* * *}$ & -3.220 \\
\hline & $(3.497)$ & $(4.969)$ & $(4.882)$ & $(5.478)$ & $(2.924)$ & $(4.079)$ \\
\hline \multirow[t]{2}{*}{ Constant } & & 10.526 & & -11.604 & & 3.318 \\
\hline & & $(6.561)$ & & $(12.278)$ & & $(5.933)$ \\
\hline R2 & & 0.393 & & 0.449 & & 0.358 \\
\hline Number of observations & & 543 & & 154 & & 697 \\
\hline Mean price charged & & 27.327 & & 32.740 & & 28.524 \\
\hline SD & & 26.079 & & 28.592 & & 26.729 \\
\hline
\end{tabular}

Notes: *** Significant at 1\%, ** Significant at 5\%, * Significant at 10\%. Robust standard errors clustered at the market level are in parenthesis. Observations are

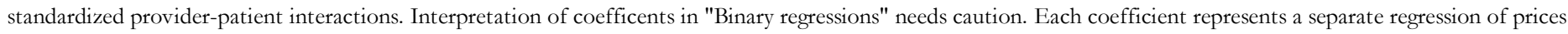

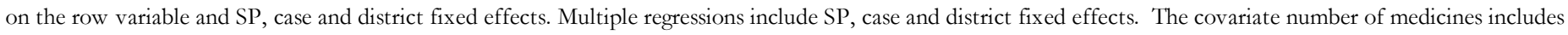
all medicines recommended to the patient (dispensed and/or prescribed). 
Table 7: Wages in the public sector (public observations only)

\begin{tabular}{|c|c|c|c|c|}
\hline & \multirow{2}{*}{\multicolumn{2}{|c|}{$\begin{array}{c}\mathbf{( 1 )}(2) \\
\text { Log of Monthly Salary } \\
\text { (pooled sample) }\end{array}$}} & \multirow{2}{*}{\multicolumn{2}{|c|}{$\begin{array}{c}(3) \quad(4) \\
\text { Desirability index } \\
\text { (PHC/CHC sample) }\end{array}$}} \\
\hline & & & & \\
\hline & $\begin{array}{c}\text { Binary } \\
\text { regressions }\end{array}$ & $\begin{array}{l}\text { Multiple } \\
\text { regression }\end{array}$ & $\begin{array}{c}\text { Binary } \\
\text { regressions }\end{array}$ & $\begin{array}{l}\text { Multiple } \\
\text { regression }\end{array}$ \\
\hline \multirow[t]{2}{*}{ Percentage of checklist items } & 0.002 & -0.001 & 0.005 & 0.004 \\
\hline & $(0.003)$ & $(0.002)$ & $(0.009)$ & $(0.009)$ \\
\hline \multirow[t]{2}{*}{ Time spent with SP (minutes) } & $-0.049 *$ & -0.010 & -0.056 & -0.076 \\
\hline & $(0.025)$ & $(0.014)$ & $(0.073)$ & $(0.076)$ \\
\hline \multirow[t]{2}{*}{ Correct Treatment } & 0.011 & $-0.097 * *$ & -0.268 & -0.146 \\
\hline & $(0.059)$ & $(0.048)$ & $(0.235)$ & $(0.201)$ \\
\hline \multirow[t]{2}{*}{ Has MBBS } & $1.056^{* * *}$ & $1.289 * * *$ & & \\
\hline & $(0.168)$ & $(0.175)$ & & \\
\hline \multirow[t]{2}{*}{ Has some qualification } & -0.094 & $0.851^{* * *}$ & & \\
\hline & $(0.367)$ & $(0.301)$ & & \\
\hline \multirow[t]{2}{*}{ Age of provider } & $0.011^{* *}$ & $0.018^{* * *}$ & $0.052^{* * *}$ & $0.060 * *$ \\
\hline & $(0.006)$ & $(0.006)$ & $(0.019)$ & $(0.024)$ \\
\hline \multirow[t]{2}{*}{ Gender of provider ( $1=$ Male $)$} & 0.114 & 0.128 & -0.580 & -0.887 \\
\hline & $(0.188)$ & $(0.106)$ & $(0.509)$ & $(0.739)$ \\
\hline \multirow[t]{2}{*}{ Born in same district } & $-0.384 * * *$ & 0.020 & -0.178 & 0.113 \\
\hline & $(0.146)$ & $(0.081)$ & $(0.444)$ & $(0.431)$ \\
\hline \multirow[t]{2}{*}{ Is a dual provider } & $0.578^{* * *}$ & $0.145^{*}$ & 0.021 & -0.169 \\
\hline & $(0.135)$ & $(0.085)$ & $(0.402)$ & $(0.523)$ \\
\hline \multirow[t]{2}{*}{ Constant } & & $8.056^{* * *}$ & & -1.361 \\
\hline & & $(0.315)$ & & $(1.188)$ \\
\hline $\mathrm{R} 2$ & & 0.623 & & 0.155 \\
\hline Number of observations & & 302 & & 183 \\
\hline
\end{tabular}

Notes: *** Significant at $1 \%$, ** Significant at $5 \%$, Significant at $10 \%$. Robust standard errors clustered at the market level are in parenthesis. The desirability index is a constructed using principal component analysis of proximity to several amenities (paved road, bus stop, railway station, Internet, post-office and bank), availability of infrastructure (stethoscope, spyghamometer, torchlight, w eighing scale, hand washing facility, drinking water, staff toilet, patient toilet, fridge, sterilizers, electric connection, electric supply, power generator, telephone, computer, IV drip, cots/beds, disposable syringes), and PHC size (number of staff and number of patients). In binary regressions columns, each coefficient represents a separate regression of prices on the row variable, a constant and district fixed effects. Multiple regressions include district fixed effects. 
Table 8: Real patients in the public and private sectors

\begin{tabular}{|c|c|c|c|c|c|c|c|c|c|c|}
\hline & (1) & (2) & (3) & (4) & (5) & (6) & (7) & (8) & (9) & (10) \\
\hline & \multicolumn{5}{|c|}{ Representative sample } & \multicolumn{5}{|c|}{ Dual sample } \\
\hline & $\begin{array}{l}\text { Time spent } \\
\text { (mins) }\end{array}$ & $\begin{array}{c}\text { Total } \\
\text { questions }\end{array}$ & $\begin{array}{c}\text { Physical } \\
\text { examination }\end{array}$ & $\begin{array}{r}\text { Dispensed/ } \\
\text { prescribed } \\
\text { medicines }\end{array}$ & $\begin{array}{c}\text { Number of } \\
\text { medicines }\end{array}$ & $\begin{array}{l}\text { Time spent } \\
\text { (mins) }\end{array}$ & $\begin{array}{c}\text { Total } \\
\text { questions }\end{array}$ & $\begin{array}{c}\text { Physical } \\
\text { examination }\end{array}$ & $\begin{array}{l}\text { Dispensed/ } \\
\text { prescribed } \\
\text { medicines }\end{array}$ & $\begin{array}{l}\text { Number of } \\
\text { medicines }\end{array}$ \\
\hline \multicolumn{11}{|c|}{ Panel A: no patient controls or fixed effects } \\
\hline Is a private provider & $\begin{array}{c}1.456^{* * *} \\
(0.323)\end{array}$ & $\begin{array}{c}0.799^{* * * *} \\
(0.180)\end{array}$ & $\begin{array}{c}0.371^{* * * *} \\
(0.108)\end{array}$ & $\begin{array}{c}-0.026^{* *} \\
(0.011)\end{array}$ & $\begin{array}{c}0.500^{* * *} \\
(0.121)\end{array}$ & $\begin{array}{c}1.894 * * * \\
(0.569)\end{array}$ & $\begin{array}{c}1.154^{* * * *} \\
(0.318)\end{array}$ & $\begin{array}{c}0.143^{* *} \\
(0.063)\end{array}$ & $\begin{array}{l}-0.008 \\
(0.009)\end{array}$ & $\begin{array}{l}-0.021 \\
(0.134)\end{array}$ \\
\hline R-squared & 0.054 & 0.030 & 0.103 & 0.003 & 0.017 & 0.115 & 0.082 & 0.017 & 0.001 & 0.000 \\
\hline Number of observations & 1,137 & 1,137 & 1,133 & 1,138 & 1,138 & 1,085 & 1,083 & 1,082 & 1,090 & 1,090 \\
\hline Mean of public & 2.378 & 2.994 & 0.473 & 0.994 & 2.319 & 1.499 & 3.284 & 0.678 & 0.991 & 3.190 \\
\hline Mean of private & 3.833 & 3.793 & 0.844 & 0.968 & 2.819 & 3.393 & 4.439 & 0.821 & 0.983 & 3.169 \\
\hline Mean of sample & 3.621 & 3.676 & 0.790 & 0.972 & 2.746 & 1.899 & 3.527 & 0.708 & 0.989 & 3.185 \\
\hline Number of public providers & 29 & 29 & 29 & 29 & 29 & 51 & 51 & 51 & 51 & 51 \\
\hline Number of private providers & 169 & 169 & 169 & 169 & 169 & 40 & 40 & 41 & 41 & 41 \\
\hline \multicolumn{11}{|c|}{ Panel B: including patient controls and market/district fixed effects } \\
\hline Is a private provider & $\begin{array}{c}1.192^{* * *} \\
(0.325)\end{array}$ & $\begin{array}{c}0.615^{* *} \\
(0.248)\end{array}$ & $\begin{array}{c}0.510^{* * *} \\
(0.086)\end{array}$ & $\begin{array}{c}0.009 \\
(0.014)\end{array}$ & $\begin{array}{c}0.548^{* * *} \\
(0.150)\end{array}$ & $\begin{array}{c}1.464^{* * * *} \\
(0.300)\end{array}$ & $\begin{array}{c}0.494^{* * *} \\
(0.128)\end{array}$ & $\begin{array}{c}0.080^{* *} \\
(0.039)\end{array}$ & $\begin{array}{l}-0.016 \\
(0.012)\end{array}$ & $\begin{array}{l}-0.014 \\
(0.098)\end{array}$ \\
\hline Has MBBS degree & $\begin{array}{l}-0.427 \\
(0.516)\end{array}$ & $\begin{array}{l}0.416^{*} \\
(0.227)\end{array}$ & $\begin{array}{c}0.155^{* *} \\
(0.079)\end{array}$ & $\begin{array}{l}-0.024 \\
(0.015)\end{array}$ & $\begin{array}{l}-0.336 \\
(0.211)\end{array}$ & & & & & \\
\hline Has some qualification & $\begin{array}{c}0.349 \\
(0.388)\end{array}$ & $\begin{array}{c}0.021 \\
(0.143)\end{array}$ & $\begin{array}{c}0.004 \\
(0.050)\end{array}$ & $\begin{array}{c}-0.034 * * \\
(0.015)\end{array}$ & $\begin{array}{l}-0.157 \\
(0.139)\end{array}$ & & & & & \\
\hline Age of Provider & $\begin{array}{c}-0.027 * * \\
(0.010)\end{array}$ & $\begin{array}{c}0.006 \\
(0.005)\end{array}$ & $\begin{array}{c}0.001 \\
(0.002)\end{array}$ & $\begin{array}{c}0.001 \\
(0.001)\end{array}$ & $\begin{array}{c}0.007 \\
(0.006)\end{array}$ & $\begin{array}{l}-0.004 \\
(0.006)\end{array}$ & $\begin{array}{c}-0.011^{* *} \\
(0.005)\end{array}$ & $\begin{array}{l}-0.001 \\
(0.002)\end{array}$ & $\begin{array}{l}-0.000 \\
(0.000)\end{array}$ & $\begin{array}{c}-0.014 * * * \\
(0.004)\end{array}$ \\
\hline Gender of Provider ( $1=$ Male $)$ & $\begin{array}{c}-1.433^{* *} \\
(0.688)\end{array}$ & $\begin{array}{l}-0.780 \\
(0.756)\end{array}$ & $\begin{array}{c}0.016 \\
(0.087)\end{array}$ & $\begin{array}{c}0.006 \\
(0.019)\end{array}$ & $\begin{array}{l}-0.116 \\
(0.203)\end{array}$ & $\begin{array}{l}-0.362^{*} \\
(0.202)\end{array}$ & $\begin{array}{c}0.056 \\
(0.203)\end{array}$ & $\begin{array}{c}-0.102 * \\
(0.054)\end{array}$ & $\begin{array}{c}0.008 \\
(0.015)\end{array}$ & $\begin{array}{l}-0.017 \\
(0.139)\end{array}$ \\
\hline R-squared & 0.295 & 0.331 & 0.368 & 0.113 & 0.309 & 0.167 & 0.354 & 0.195 & 0.051 & 0.180 \\
\hline Number of observations & 835 & 835 & 833 & 835 & 835 & 812 & 811 & 810 & 813 & 813 \\
\hline
\end{tabular}

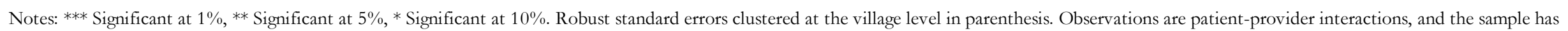

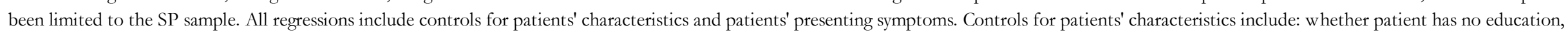

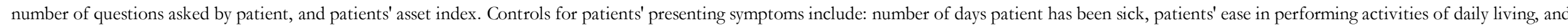

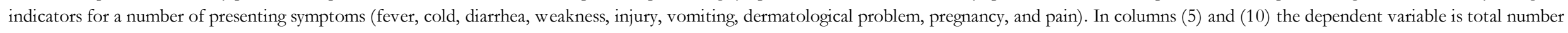
of medicines recommended to the patient (dispensed and/or prescribed). 


\section{Appendices (Text, Figures, Tables)}

\section{A Mapping and Sampling of Providers}

\section{A.1 Mapping of Providers: Representative Sample}

We first randomly selected five districts in the state of Madhya Pradesh, stratified by region and an index of health outcomes. In each district, we sampled 20 villages by probability proportional to size (PPS) sampling. Because of the rural focus of the study, we restricted the sampling frame to villages with populations under 5,000. The sample of villages is thus representative of rural Madhya Pradesh.

In each sampled village, we conducted at least three Participatory Resource Assessments in different locations within the village and obtained a list of all the healthcare providers that households' sought primary care services from. These lists were used primarily to identify the geographical locations that households' sought care from. For instance, households may seek care from providers within the village, but also on the nearest highway. If 5 percent or more households reported visiting a provider in an outside location, we identified that location as a "cluster village" and considered it a part of the "healthcare market" for the sampled village. Fifty-five sampled villages have one cluster village, 13 villages have two, and one village has three. The remaining 31 villages have no cluster villages (i.e. less than 5 percent of primary healthcare visits were to a location outside the village). For our sample as a whole, we identified 184 unique locations, including the 100 sampled villages.

Surveyors then visited each location and administered a provider census to all healthcare providers in the location - regardless of whether they had been mentioned in the participatory assessments. The provider census details the provider's demographic, practice and clinic characteristics.

Following the provider census in the villages, we administered a short household census and obtained information on household demographics and healthcare seeking behavior. For each household member, we asked about incidence of any illness (primary or otherwise) in the past one month, if they sought medical attention for that illness, and (if yes), the name and address of the provider they visited (regardless of the location of the provider). Surveyors mapped the household visits to the providers lists; this is the mapping we use to compute the fraction of visits to public and private providers and providers with different qualifications

in Table 1. In instances where households reported visiting providers not already on the list, we probed for providers' name, address and practice details and added the providers to our listing and census exercise. We verified through this exercise that we had covered 
at least 95 percent of all providers visited by households in each village. This exhaustive mapping process ensured that we mapped the complete "health market" where households in our sampled villages sought primary care services.

\section{A.2 Sampling of Providers for SP visits: Representative Sample}

We conducted the SP work in three out of the five districts in our sample because of logistic considerations. Although SPs were recruited from the local community, they needed plausible reasons for their presence in the village (which they were not from), and the typical narrative was that they were traveling and/or passing through the village. In order to minimize SP detection, we excluded 5 remote markets (as assessed by road access) from the possible 60 markets, where we thought a traveling excuse might not be plausible.

We sampled providers for the SPs to visit from a smaller set of "eligible providers" than what we had mapped. All public nurses and midwives (ANMs), community health workers (ASHA), and day-care center workers (Anganwadi), were excluded from the sample as they provided primarily preventive care such as vaccinations. We also exclude mobile and itinerant providers, chemists, and pharmacists from the sample. Finally, we exclude 55 providers with whom we could not complete the provider census prior to sampling (typically due to the unavailability of provider, we were able to conduct the census with only 17 of these providers in subsequent rounds). These restrictions remove 7 markets from our study, primarily because there were no eligible providers in these markets. We also drop two other markets because they share a cluster with other sampled villages and do not have eligible providers inside the village. Our study in the representative sample therefore covers 46 markets in 3 districts of Madhya Pradesh (see Table A.1). Based on the eligibility criteria defined above, these 46 markets have 649 eligible providers (130 public and 519 private) from which we sample.

In each market we randomly sampled up to two eligible providers in each public clinic and up to six private providers in each market. ${ }^{1}$ In the private sector, we sampled one provider per clinic. We also sampled all MBBS providers in both public and private sectors. Since the unit of analysis for the representative sample is the clinic and not the provider, this sampling procedure was equivalent to sampling private clinics with simple random sampling (after sampling all private MBBS doctors), and sampling larger public clinics (those with two or more eligible providers) twice. We sampled a total of 247 providers of which 45 are public providers and 202 are private providers (Appendix Table A.1).

\footnotetext{
${ }^{1}$ One market in Gwalior district was an exception to this rule. In the cluster village of a particular market, we found 113 providers. In this market, we relaxed our sampling protocol and sampled 20 private providers.
} 


\section{A.3 Completion of SPs: Representative Sample}

We sampled 247 providers in 235 clinics and SPs were completed with 224 providers in 215 clinics. Of these, 214 providers are those we sampled. Furthermore, for $27 \mathrm{SP}$ interactions (corresponding to 10 providers) we saw a provider who had not been sampled. We knew the identity of the provider because he/she had been included in the census but was practicing in a clinic different from their own. For 18 observations (corresponding to 8 public and 2 private clinics and 10 public and 2 private providers sampled) we do not know the identity of the provider. These were most likely staff present in the clinic who are not licensed to provide care, but who do so when the doctor is absent.

The discrepancy between whom we sampled and whom we actually saw does not affect interpretation of our results in Panels A and B of Tables 3-5, but it does in Panel C, where we include controls for provider characteristics. Panels A and B present results without provider controls, so whether or not we have background data on the provider is irrelevant, because we know which market they were practicing in and whether they were public or private. This is why the public-private difference here should be interpreted as the difference in random visits to providers' clinics rather than providers. In Panel $\mathrm{C}$, we present results including provider controls. Here, for 27 interactions where we saw providers we did not sample but mapped (and conducted provider census), we use their background information. The 18 observations where we do not know the provider at all are dropped from the estimation sample.

\section{A.4 Mapping of Providers: Dual Practice Sample}

We obtained a list of all Primary Health Centers (PHCs) and Community Health Centers (CHCs) from the Ministry of Health of Madhya Pradesh. Excluding PHCs/CHCs which were mapped as part of the representative sample, we mapped 200 more facilities in this round. Of these 200 facilities, 40 did not have an MBBS provider posted (see Appendix Table A.2). In the remaining 160 PHCs/CHCs we located 216 providers (some providers were mapped to multiple facilities). Our field team then undertook detailed field work to find out if these providers operated private practices and if yes, to locate their private practices. We were able to locate a private practice for 132 of the 216 providers (61.1 percent) (this is the sample we call the "dual practice sample"). After the mapping, we administered the provider census to all providers. To the extent possible, the census was administered in the private clinic of the provider. 


\section{A.5 Sampling of Providers: Dual Practice Sample}

We sampled one MBBS doctor from every PHC/CHC with preference for one with a dual practice there were multiple MBBS doctors in the clinic. In cases where a provider was posted to multiple public facilities, and where there were no additional MBBS providers in these facilities, we randomly sampled the provider from one of the multiple facilities they were posted to. With this sampling strategy, we sampled from 143 of the 160 facilities we could have sampled from. Of the 143 providers, 94 operated private practices (65.7 percent, see Table A.2).

\section{A.6 Completion of SPs Interactions}

SPs completed interactions with 118 of the 143 providers sampled, primarily because providers were absent or were away on "long leave" in the 6-month phase between the listing and the SP work. We attempted to complete these providers over a minimum of 4 attempts and were forced to stop trying at that point due to the heightened risk of detection. Of the 49 providers without private facilities, SPs completed interactions with 30 providers (61.2 percent). Of the 94 providers with private practices, SPs were able to complete at least one interaction with 88 providers (93.6 percent, either public or private). The number of dual practice providers for whom we have at least one observation in both their public and private practice is 69. As discussed in text, it was more difficult to complete a case in the public practice of the public MBBS doctors (because of high absence rates), and we had a lower completion rate in the public practices of these doctors than in the private practices (75 percent versus 93 percent).

\section{B Standardized Patient Data Collection and Notes}

\section{B.1 Description of Tracer Conditions and Relevance for India}

SPs presented either a case of unstable angina, asthma, or dysentery of an absent child.

- Unstable Angina: A 45-year-old male complains of chest pain the previous night. Appropriate history taking would reveal classic signs (radiating, crushing pain) and risk factors (smoking, untreated diabetes, and family history of cardiac illness) of unstable angina or an imminent myocardial infarction.

- Asthma: A 25-year-old male or female presents with difficulty breathing the night before the visit. When questioned appropriately, the SP reveals that the episode lasted for 10 to 15 minutes and involved a "whistling" sound (wheezing) and that he or she 
has had similar episodes before, often triggered by house cleaning and cooking smoke. The SP also reports a family history of similar symptoms.

- Dysentery: A 26-year-old father of a 2-year-old complains that his child has diarrhea and requests medicines. When probed, the SP reveals details of their water source and sanitation habits, in addition to the presence of fever and the frequency and quality of the child's stools.

For all cases, checklists of recommended history questions and examinations were developed together with an advisory committee and SPs were trained to recall the questions asked and examinations performed. These were then recorded during debriefing with the supervisor using a structured questionnaire within an hour of the interaction. In a recent study, we test the reliability of recall by comparing audio recordings with recall and find a very high correlation of $0.63(\mathrm{p}<.001)$ (Das et al., 2015).

\section{B.2 Relevance of Cases}

Incidence of cardiovascular and respiratory diseases has been increasing, and diarrheal disease kills more than 200,000 children per year in India (Black et al., 2010; Patel et al., 2011) The Indian government's National Rural Health Mission (NRHM) has developed triage, management, and treatment protocols for unstable angina, asthma, and dysentery in public clinics, suggesting clear guidelines for patients presenting with any of these conditions (Jindal et al., 2005). The cases were also chosen to minimize risk to standardized patients since they could not portray any symptoms of infection given the documented high propensity to administer medicines intravenously with unsterilized needles and to use thermometers that have not been appropriately disinfected (Banerjee, Deaton and Duflo, 2004).

\section{B.3 SP Recruitment, Script Development and Training}

22 SPs were recruited from an initial group of 45 who were extensively screened and trained for 3 weeks. The age and sex of recruited SPs corresponded to the relevant tracer conditions. For instance, angina was depicted by male SPs between 40 and 50 years old.

Scripts were developed under the guidance of a medical anthropologist with active SP participation that described the social and family contexts of the patient if a provider were to ask questions about these details. Script development and SP training jointly ensured that the clinical symptoms and case history reflected the social and cultural milieu of which the SP was assumed to be a member and, second, the presentation of symptoms and answers to history were consistent with biomedical facts about the disease. SPs were trained to present 
symptoms and answer questions pertaining to case history that were medically correct. For example all opening statements and questions pertaining to the type of cough and its duration were standardized. SPs were also trained to distinguish between questions to which answers could be improvised but had to be appropriate to the social role of the SP and answers that had to be given using local idioms but in a standardized format without any alterations.

All SPs underwent rigorous training for 100-150 hours that started with a focus on the cases and the development of scripts and proceeded to memorization and appropriate roleplaying, as well as techniques to perfect recall of the questions asked and examinations completed during the interaction. Following the training, SPs visited doctors who were working with our team to provide feedback on their presentation and depiction of the cases. Finally, dry runs were completed with unannounced visits to consented providers to help build the confidence of the SPs and take them through a number of "real-life" situations. Field work started once protocols were in place for the variety of these experiences.

With consent from the Institutional Review Board at Harvard University, the study was first piloted in Delhi with 64 consented providers who had been previously informed that they would be visited by an SP within the next 6 months (see Das et al. (2012)). In the pilot phase of the study, a total of 248 out of a potential 256 SP interactions were completed. Within a month of the SP visit, field-workers visited the consented providers to enquire if they had been visited by an SP. In cases where the provider felt that an SP visit had occurred, we elicited the sex, approximate age and symptoms of the SP. We could confirm a match between the providers' suspicions and the actual SP sent to the provider in only 2 cases for a detection rate of less than 1 percent.

The Institutional Review Boards of Innovations for Poverty Action and the Central and State governments in India granted clearance for the study. To minimize detection in rural Madhya Pradesh, where providers are more likely to recognize their entire patient population, the study proceeded as an audit, and providers were not aware that they were being visited by standardized patients. Clearance was granted for this deception design because the risks to providers and their patients were minimal, whereas accurate measures of provider practice were nonexistent. The expected length of clinical interactions, patient loads, and levels of provider anxiety induced by the cases were thought to be small, and standardized patients had to pay providers whatever they charged. The waiver of consent is consistent with the principle that where the research subject provides a public service to other customers, the public have a right to know about the quality of the service provided (Norris, 2002). 


\section{B.4 Categorizing Treatment in SP Interactions}

In rural Madhya Pradesh, as in much of India, providers often dispense medicines in the clinic rather than prescribe them for purchase from external chemists (some do both). Our field staff recorded names of all dispensed/prescribed medicines in SP exit interviews and used multiple resources to classify medicines as accurately as possible. Field staff were given a list of commonly used drugs in India along with their medical classification, and the CIMS Drug Information System (in print), which they used to record exact medicine names and classes. For drugs that were not immediately confirmed, they consulted local chemists and pharmacists and obtained correct names to the extent possible.

To construct our main treatment variables - correct treatment, palliative treatment and unnecessary/harmful treatment - we obtained from a panel of doctors in the United States and India a full list of correct and palliative treatments/medicines for each case. These include nitrates, aspirin, clopidogrel, anti-platelet agents, blood thinners, beta blocker, morphine, other pain control, ACE inhibitor and vasodilator for unstable angina; ORS, electrolytes and zinc for dysentery; and inhaled-corticosteroids, leukotriene inhibitors, cromones, inhaled-anticholinergics and oral-corticosteroids for asthma (see Table A4).

After medicine coding in the field, the authors and members of the ISERDD team in Delhi verified the codes assigned to all medicines and recoded if them when necessary. To further ensure the coding was correct, we used a third party, a pharmaceutical consulting firm in Delhi, to independently verify our classification of medicines.

Medicine coding is relatively straightforward in instances where providers prescribe and SPs receive a written prescription. In cases where providers dispense, it was easier to obtain names when medicines came with packaging than when they did not. In the 1,123 complete SP interactions, SPs were recommended a total of 2,772 medicines corresponding to 969 unique medicines (by medicine names, ignoring unlabeled ones). We are unable to classify 14.18 percent of the all 2,772 medicines because they were unlabeled (providers dispensed them as loose samples or in crushed powder form). We are further unable to classify 3.64 percent of medicines (93 unique medicines by name) because we could not match them to secondary information sources. SPs received at least one unclassifiable medicine in 268 interactions (23.9 percent of all interactions). However, in 211 of these interactions (18.8 percent), SPs received classifiable medicines along with the unclassifiable medicines. In only 57 interactions (5.1 percent) were all medicines unclassifiable.

We construct our main treatment variables - correct treatment, palliative treatment and unnecessary treatment - after completing the medicine coding process described above. For each interaction, we determine if any recommended medicines fall into correct, palliative 
and/or unnecessary treatments, treating all unlabeled and unidentifiable medicines as unnecessary. It is possible that the unlabeled and unidentifiable medicines construe correct or palliative treatment. However, the likelihood that the provider dispenses an unclassifiable medicine is decreasing in other measures of provider quality from the SP study. We are therefore confident that such medicines are more likely unnecessary treatments than not. Our results are also robust to excluding interactions that include unclassifiable medicines.

\section{Theoretical Appendix}

We provide a simple theoretical framework which demonstrates providers' choice of effort and treatment with and without market incentives as well as the effects of their choices on a patient's health outcome. This framework incorporates three possible channels which can generate excessive unnecessary treatments. The first channel is ignorance. Providers want to treat patients, but they do not know the cause of patient's symptoms and dispense a cocktail of medicines hoping that one of the medicines would work. Second, there is a pecuniary incentive to sell more medicines. Third, excessive treatment can be driven by patients. Patients have their own expectation about proper treatment, and providers may satisfy patients by meeting their demand for medication.

One key insight from the framework is that unnecessary treatments are not only driven by market incentives but can also arise due to low effort of providers. When providers lack motivation to exert enough effort to substantially reduce their ignorance about a patient's condition, they may provide multiple treatments to increase the chance of providing a correct one. Market incentives induce higher effort (and a more precise diagnosis) but also lead to more unnecessary treatment at any given level of effort. ${ }^{2}$ Hence health outcomes produced under a market system may not necessarily dominate that of public system, and vice versa.

Our aim is to provide a framework which helps to interpret the empirical findings related to the choice of effort and treatment by providers facing different incentives. We abstract from any market equilibrium component such as pricing, entry and exit decisions of providers, or any strategic interaction among providers in the market or across sectors (public and private). Patients' expectations also enter exogenously. We also assume that private providers have dynamic incentives to acquire a positive reputation, but do not endogenize these market incentives since a static framework is adequate to interpret our empirical findings. A theoretical extension where we provide one potential way of endogenizing market incentives is available on request, but is omitted here because our data does not allow us to study the dynamics of reputation and price setting.

\footnotetext{
${ }^{2}$ This can also explain why we do not see a higher level of over-treatment by private providers in the dual sample, even though we find that there is a marginal incentive to over-treat.
} 


\section{C.1 Problem Setup}

A patient visits a provider endowed with a level of medical knowledge $K$. The patient has an illness defined by the required type of treatment denoted by $n^{\text {true }}$. Patients with different underlying illnesses may experience similar symptoms. In other words, given a set of symptoms, there is a distribution of $n^{\text {true }}$ associated with the symptoms. A provider's job is to identify the true state of the patient and perform adequate treatments. The provider-patient transaction is modeled as a two-stage process: consultation and treatment. A subscript $i$ for $i^{t h}$ provider is used when there is a need to emphasize heterogeneity among providers. The subscript is suppressed otherwise for notational simplicity.

\section{C.2 Consultation Stage}

A patient visits a provider. The true state of the patient $n^{\text {true }}$ is unobserved to both the patient and the provider. The patient describes her symptoms, and the provider forms a prior belief about the true state given the described symptoms. The prior belief follows a normal distribution:

$$
n^{\text {prior }} \sim N\left(\nu, \frac{1}{\alpha}\right)
$$

The prior belief can be thought of as the distribution of illnesses in the region which cause the given symptoms. The provider exerts costly effort $e$ to learn about $n^{\text {true }}$. The cost of effort is given by $e^{2}$. We can interpret $e$ as the number of checklist items completed by the provider or time spent with the patient. $e$ can also be observed by the patient. The provider draws a noisy signal $s \sim N\left(n^{\text {true }}, \frac{1}{\beta}\right)$ by exerting $e$ where $\beta=e K$. The signal is not observed by the patient. Given $s$, the provider updates his belief about $n^{\text {true }}$. The posterior belief of the true state is given by:

$$
n^{\text {post }} \sim N(\underbrace{\frac{\alpha \nu}{\alpha+\beta}+\frac{\beta s}{\alpha+\beta}}_{\equiv \mu}, \frac{1}{\alpha+\beta})
$$

This is the result of standard Bayesian normal updating, and hence, a separate proof is omitted. Note that $n^{\text {post }} \rightarrow n^{\text {true }}$ as $\beta \rightarrow \infty$.

\section{C.3 Treatment Stage}

Based on the posterior belief about the true state, the provider decides the types of treatment he will perform. The treatment is expressed as an interval $[\mu-n, \mu+n]$, and $n$ is interpreted as the variety of treatments chosen by the provider. Let $F_{e}$ denote the cumulative density 
function of the posterior belief given some level of effort $e$. Given $K$, the shape of the posterior belief is governed by $e$ ( $e$ and $\beta$ are used interchangeably depending on the context). The probability that the interval $[\mu-n, \mu+n]$ includes $n^{\text {true }}$ is denoted by $P_{e}(n)$ where $P_{e}(n)=F_{e}(\mu+n)-F_{e}(\mu-n)$. There is a health cost of using multiple treatments given by $n^{2}$. The expected health outcome, $H$, is a function of $e$ and $n$ and is given by $H(e, n)=P_{e}(n)-n^{2}$. Note that for each individual patient, the interval either includes the true state or not with probability of $P_{e}(n)$ and $1-P_{e}(n)$.

The patient has her own belief about the proper treatment that she expects to receive when visiting a clinic given the symptoms she has, which is denoted by $\bar{n}$. It is assumed that $\bar{n}$ is also known to the provider. When the chosen $n$ is different from $\bar{n}$, the provider needs to communicate with the patient to convince her that his choice of $n$ is the correct treatment. The farther away $n$ is from $\bar{n}$, the greater the need for communication. Also, the patient can be more easily convinced if the provider has exerted more effort to examine her

in the first place. The cost of communication is given by $\frac{(n-\bar{n})^{2}}{e}$. An easy way to reduce this communication cost is to simply give something close to $\bar{n}$. We are particularly interested in the case where $\bar{n}$ is large.

\section{C.4 Providers' Optimization Problem with and without Market Incentives}

Denote the maximized utility of providers in the consultation stage and treatment stage by $V_{1}$ and $V_{2}$ respectively. Without market incentives, providers have low-powered incentives and maximize their utility:

$$
\begin{gathered}
V_{1}=\max _{e}\left\{-e^{2}+V_{2}(e)\right\} \\
V_{2}(e)=\max _{n}\left\{\phi H-\frac{(n-\bar{n})^{2}}{e}\right\}
\end{gathered}
$$

where $\phi$ governs the extent to which providers care about patients' health without highpowered incentives.

In market environment, providers face market incentives in addition to low-powered incentives. Now, a provider $i$ charges a piece rate $\tau_{i}$ per unit of effort as a consultation fee and also charges $p$ per unit of $n$ for the treatment. Providers also care about their reputation in the market, which is determined by the health outcomes of their patients. Health outcomes are not fully observed in the market because the long-term health cost of excessive treatment is not as easily observed as the immediate relief of symptoms. Instead, reputation is based on the observed health outcome $H^{o}$, which is given by $H^{o}(e, n)=P_{e}(n)-\gamma_{o} n^{2}$ where $0<\gamma_{o}<1$, and $\delta$, which is a parameter that governs the extent to which providers care about their reputation in the market. When there are market incentives, providers maximize their utility 
given by:

$$
\begin{gathered}
V_{1}(\tau)=\max _{e}\left\{-e^{2}+\tau e+V_{2}(e)\right\} \\
V_{2}(e)=\max _{n}\left\{\phi H+\delta H^{o}-\frac{(n-\bar{n})^{2}}{e}+n p\right\}
\end{gathered}
$$

To focus on how the presence of market incentives shapes the optimal choices of providers, we omit the patients' expectation channel and remove the term $-\frac{(n-\bar{n})^{2}}{e}$ from the provider's maximization problem. We reintroduce the term in subsection C.1.5.

The first order conditions without market incentives are given by:

$$
\begin{gathered}
\phi f_{e}(\mu+n(e)) \frac{n(e) K}{\sqrt{\alpha+e K}}=2 e \\
f_{e}(\mu+n)=n
\end{gathered}
$$

where $f_{e}$ is the probability density function of the posterior belief given $e$. The term $f_{e}(\mu+n)$ captures the marginal benefit of increasing $n$ through the higher probability of spanning the correct treatment, and the right hand side is the marginal cost of increasing $n$ through the higher health cost of excessive treatment. In the absence of market incentives, note that providers choose $n$ which maximizes $H$ at any given $e$.

The first order condition in the consultation stage with market incentives is given by:

$$
\tau+(\phi+\delta) f_{e}(\mu+n(e)) \frac{n(e) K}{\sqrt{\alpha+e K}}=2 e
$$

and the first order condition in the treatment stage is given by:

$$
f_{e}(\mu+n)+\frac{p}{2(\phi+\delta)}=\left(\frac{\phi+\gamma_{o} \delta}{\phi+\delta}\right) n
$$

It is easy to see from (4) and (6) that given $e$, providers choose larger $n$ when there are market incentives. Because there is pecuniary benefit from $n$ and also because the cost of excessive $n$ is not fully observed in the market $\left(\gamma_{o}<1\right)$, given $e$, the marginal benefit of $n$ is always greater and the marginal cost is always smaller with market incentives. Thus, providers choose excessive $n$ where $H$ is decreasing in $n$ instead of where $H$ is maximized. This means that by slightly decreasing $n$, the health outcome can be improved.

Whether market incentives induce higher effort depends on the relative size of the rewards for $e$ and $n$ in the market. As long as the rewards for $n$ are not so large so as to dominate 
those for $e$, providers choose higher $e$ with market incentives. ${ }^{3}$ Since our empirical results find that private providers always exert more effort (in both the representative and dual samples) and we also find a robust positive relationship between prices charged and effort expended, it appears that the $\tau$ in our setting is high enough to induce additional effort from providers facing market incentives.

\section{C.5 Market Incentives and Health Outcomes}

However, even if provider effort were to always be higher under market incentives, the impact of market incentives on health outcomes is ambiguous and will depend on parameter values. In particular, when $\phi$ is very low, it is possible that health outcome under market incentives are better; however, as $\phi$ increases, health outcome without market incentives may be better. ${ }^{4}$

Figures 1 and 2 in the main text illustrate this mechanism. Panel (A) in Figure 1 illustrates a case where market incentives induce higher effort. $M B_{\text {with }}$ and $M B_{\text {without }}$ are the left hand side of (5) and (3) with respect to e. $M C_{\text {with }}$ and $M C_{\text {without }}$ are the right hand side of (5) and (3) with respect to $e$. The terms $e_{\text {with }}^{*}$ and $e_{\text {without }}^{*}$ are the optimal levels of effort with and without market incentives, respectively, for small and large $\phi$ values. The rewards for effort in the market are sufficiently large in this case that $e_{\text {with }}^{*}$ is larger than

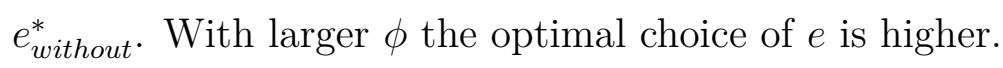

Panel (B) traces posterior variance $\frac{1}{\alpha+\beta}$, the inverse of posterior precision, as a function

\footnotetext{
${ }^{3}$ If there is little punishment for excessive treatment in the market and the marginal profit from treatment is large, providers may find it optimal to choose low $e$ and profit from large $n$ unless the rewards for effort, $\tau$, are large enough to offset the tradeoff. To see this from the first order conditions (3) and (5), observe that the marginal benefit of $e$ is larger when there are market incentives if $n(e)$ were the same for both with and without market incentives. However, providers choose larger $n$ when there are market incentives. Note that, for any given $e, n f_{e}(\mu+n)$ is increasing in $n$ when $n<\frac{1}{\sqrt{\alpha+\beta}}$, maximized when $n=\frac{1}{\sqrt{\alpha+\beta}}$, and decreasing in $n$ when $n>\frac{1}{\sqrt{\alpha+\beta}}$. Also, $n f_{e}(\mu+n)$ is bounded below by 0 and above by $\frac{1}{\sqrt{2 \pi}} \exp \left\{-\frac{1}{2}\right\}$. Let $n_{1}(e)$ and $n_{0}(e)$ denote the optimal choice of treatment as a function of $e$ with and without market incentives. Observe that when $n_{1}(e)<\frac{1}{\sqrt{\alpha+\beta}}$, the marginal benefit of $e$ with market incentives is always greater than that without market incentives. The $n_{1}(e)$ term is more likely to be smaller than $\frac{1}{\sqrt{\alpha+\beta}}$ when market incentives for $n$ are small, i.e. when $p$ is small and $H^{o}$ is close to $H$. On the other hand, when $n_{1}(e)>\frac{1}{\sqrt{\alpha+\beta}}$, the left hand side of (3) may be larger than that of (5). However, because $n f_{e}(\mu+n)$ is bounded, we can always find a $\tau$ which makes the marginal benefit of $e$ with market incentives larger.

${ }^{4}$ Although we do not provide a formal proof, we confirmed in numerical simulations that there exists a set of parameter values for which the model produces outcomes consistent with the argument provided here. There are three conditions on parameter values for the model to produce such outcome: (1) $\tau$ is large enough so that providers exert high effort even when $\phi$ is small; (2) $K$ is large enough so that exerting extra effort is meaningfully translated into higher posterior precision; (3) $p$ and $\gamma_{o}$, the parameters that govern the size of market incentives for excessive treatment, are large enough to induce excessive treatment even when $\phi$ becomes high, but also small enough to not entirely offset the gains from a more precise posterior distribution that is obtained due to market rewards for higher effort (especially at low values of $\phi$ ). The simulation results are available upon request.
} 
of $e$ holding $K$ constant. The y-intercept, $\frac{1}{\alpha}$, is the posterior variance when $e=0$. The term

$\frac{1}{\alpha+\beta}$ decreases with $e$ at diminishing rates because $\beta=e K$. When $\phi$ is small, a difference in $e$ is translated into a substantial difference in $\frac{1}{\alpha+\beta}$. When $\phi$ is large, the marginal effect of effort on $\frac{1}{\alpha+\beta}$ is small.

Panel (C) illustrates the optimal level of treatment with and without market incentives, $n_{\text {with }}^{*}$ and $n_{\text {without }}^{*}$, when the posterior variance with market incentives is substantially smaller than that without market incentives. $M B_{\text {with }}$ and $M B_{\text {without }}$ are the left hand side of (6) and (4) with respect to $n . M C_{\text {with }}$ and $M C_{\text {without }}$ are the right hand side of (6) and (4) with respect to $n$. The slope of $M C_{\text {with }}$ is smaller than 1 because the health cost of excessive treatment is not fully observed, and hence, penalties for additional treatment in the market are weaker than what providers would impose on themselves under low-powered incentives. The term $p$, the unit price of $n$, is added to $M B_{w i t h}$, so $M B_{w i t h}$ asymptotes to $\frac{p}{2(\phi+\delta)}$ rather than to 0 . When the posterior variance with market incentives is substantially smaller than that without incentives, the optimal level of $n$ with market incentives can be smaller in spite of incentives for excessive treatment. Panel (D) illustrates the optimal level of treatment when the posterior variance with market incentives is only slightly smaller than that without market incentives. In this case, the effects of market incentives on excessive treatment dominates, and the optimal level of $n$ is larger with market incentives.

Figure 2 illustrates the health outcome produced with and without market incentives with different values of $\phi$. $H$ increases with $\phi$ because $e$ increases with $\phi$, and $n$ is invariant to $\phi$ given $e$ when there are no market incentives and decreases with $\phi$ when there are market incentives. We argue that, when $\phi$ is low, $H$ is higher with market incentives; however, as $\phi$ increases, $H$ without market incentives starts to dominate that with market incentives. At low levels of $e$, a small difference in $e$ is translated into a substantial difference in the posterior precision. Although market incentives induce excessive $n$, the effect of higher posterior precision on the health outcome dominates the offsetting effect of excessive $n$. However, as $\phi$ increases, $e$ under both environment increases, and the marginal effect of $e$ on the posterior precision, and hence on the health outcome, becomes smaller. At sufficiently high levels of $e$, higher $e$ with market incentives generates a difference in the posterior precision that is too small to offset the effect of excessive $n$. Thus, when $\phi$ is high, the health outcome without market incentives is higher.

\section{C.6 Re-introducing Patient Expectations}

When patients have their own expectation about proper treatment, providers engage in costly communication to convince patients that their choice of $n$ is the correct treatment. The first order conditions with the communication cost, $\frac{(n(e)-\bar{n})^{2}}{e}$, are given below. 
Without market incentives :

$$
\begin{gathered}
\phi f_{e}(\mu+n(e)) \frac{n(e) K}{\sqrt{\alpha+e K}}+\frac{(n(e)-\bar{n})^{2}}{e^{2}}=2 e \\
f_{e}(\mu+n)=\left(1+\frac{1}{e \phi}\right) n-\frac{1}{e \phi} \bar{n}
\end{gathered}
$$

With market incentives :

$$
\begin{gathered}
\tau+(\phi+\delta) f_{e}(\mu+n(e)) \frac{n(e) K}{\sqrt{\alpha+e K}}+\frac{(n(e)-\bar{n})^{2}}{e^{2}}=2 e \\
f_{e}(\mu+n)+\frac{p}{2(\phi+\delta)}=\left(\frac{\phi+\gamma_{o} \delta}{\phi+\delta}+\frac{1}{e \phi+e \delta}\right) n-\frac{1}{e \phi+e \delta} \bar{n}
\end{gathered}
$$

Note that, in the treatment stage, the marginal cost of $n$ is smaller than that when the patients' expectation channel is omitted when $n<\bar{n}$ and it is larger when $n>\bar{n}$. Thus, there is an incentive to choose $n$ closer to $\bar{n}$. Figure C.1 illustrates the effect of some large $\bar{n}$ in the treatment stage. We consider a case where providers choose higher effort with market incentives. Panel (A) is when the patient's expectation channel is omitted and panel (B) is when the channel exists. In panel (A), $M B_{\text {with }}$ and $M B_{\text {without }}$ are the left hand sides of (6) and (4) with respect to $n . M C_{\text {with }}$ and $M C_{\text {without }}$ are the right hand sides of (6) and (4) with respect to $n$. In panel (B) they are the left and the right hand sides of (8) and (7). The optimal levels of treatment with and without market incentives are labeled as $n_{\text {with }}^{*}$ and $n_{\text {without }}^{*}$. At small values of $n, \mathrm{MC}$ of $n$ is lower in panel (B) than in (A) because the communication cost decreases as $n$ comes closer to $\bar{n}$. Thus, the optimal choice of $n$ is larger than that without patients' expectation. The effect is larger when there are no market incentives where the level of effort is lower and the communication cost of deviating from $\bar{n}$ is greater. 
Figure C.1: Optimal choice of treatment with and without patient's expectation $\bar{n}$
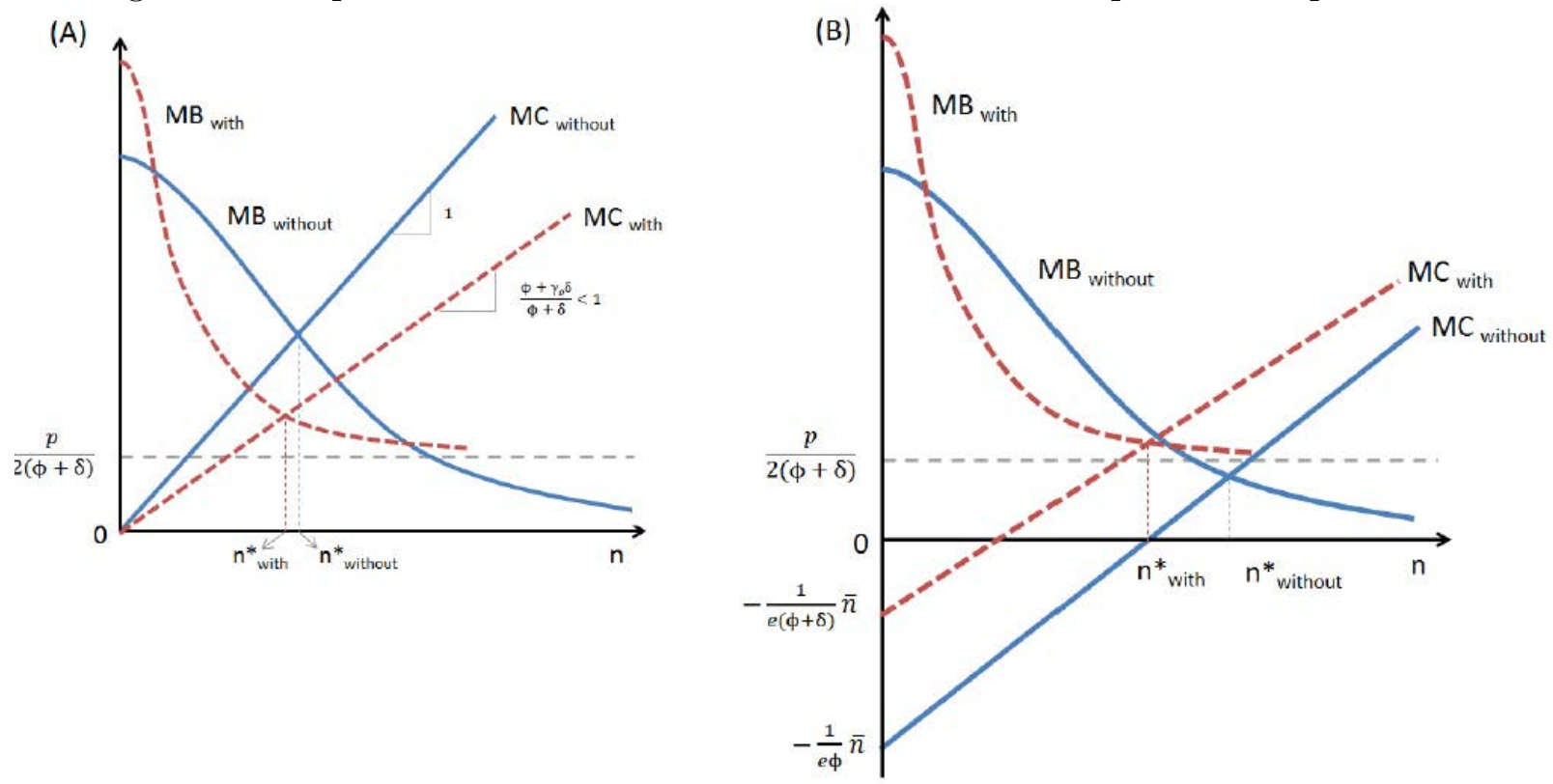

Notes: The graphs illustrate the effect of some large $\bar{n}$ when the effort with market incentives is larger than when without. Panel (A) is when the patient's expectation term is omitted and panel (B) is when it is present. In panel (A), $M B_{\text {with }}$ and $M B_{\text {without }}$ are the graphs of the left hand side of (6) and (4) with respect to $n . M C_{\text {with }}$ and $M C_{\text {without }}$ are the graphs of the right hand side of (6) and (4) with respect to $n$. In panel (B), $M B_{\text {with }}$ and $M B_{\text {without }}$ are the graphs of the left hand side of (8) and (7) with respect to $n$. $M C_{\text {with }}$ and $M C_{\text {without }}$ are the graphs of the right hand side of (8) and (7) with respect to $n$.

\section{Differential Patient Sorting Across Sectors}

Although audit studies are advantageous over other metrics of healthcare quality such as vignettes and direct observations, they are limited by the number and type of cases that can be presented. As we discussed in Section 3.1, one reason we selected our cases - unstable angina, dysentery and asthma - was that providers could clinically diagnose and recommend treatment to SPs without need for invasive procedures or examinations that could potentially harm the SPs. Then, when using SPs to measure healthcare quality, one has to worry if the cases presented represent off-equilibrium conditions for the market.

Furthermore, even if presented cases map well into the overall morbidity patterns and care seeking behaviors of the population, it is possible that patients choose to visit different provider types (public or private) for different types of conditions. Patients may choose public facilities for more serious conditions, or vice versa. Or for instance, in cases such as tuberculosis there is enormous government attention, and private providers of all stripes 
immediately refer patients to the public sector. Not accounting for such differential patient sorting across sectors will produce confounded estimates of the public-private healthcare quality gap.

Note that this is a difficult problem to address in general because observing real providerpatient interactions precludes the possibility of off-equilibrium behavior, but we cannot code the quality of care accurately because we do not know the underlying ailment. On the other hand, the SP method allows for better measurement of quality of care, but may represent an off-equilibrium interaction

Here, we provide evidence against differential patient sorting using direct observation data described in Section 7. We conducted patient exit interviews immediately after provider interactions in which we ask patients reasons for their visit including a list of symptoms, their current physical activity, and other background and demographic questions. In Table A.14 we present estimates of differences in patient characteristics in the public and private clinics. For the representative sample, for each outcome variable (rows of the table), Columns (1) and (2) present means in public and private sectors respectively, and Columns (3) and (4) present coefficients from regressing the outcome variable on a private indicator with and without market fixed effects. Our preferred estimate of public-private difference is in Column (4) and we discuss these coefficients below. Among the nine common symptoms we record, there are only two symptoms where observe significant differences in observing a symptom across sectors. Patients are 13.5 percentage points more likely to visit the private sector when they have a fever and 4.6 percentage points more likely when they experience vomiting. There is no difference in the number of days the patients has been sick before coming to see the provider. Similarly there are no significant differences between patients assets index or education levels, but patients to the private sector are more likely to have traveled from outside than patients in the public sector (which is consistent with results in table 1 that patients are more likely to travel to see a private provider).

Columns (5)-(8) repeat the same exercise for the dual sample except that we use district fixed effects instead of market fixed effects. Here, the differences in patterns of observed symptoms are even smaller and not a single outcome is statistically different across the two sectors. Patients in the private sector are richer (which is not surprising because MBBS providers charge a much higher fee) and are 8.7 percentage points (3 percent) more likely to have formal education. Finally, in both representative and dual samples we find that patients are more likely to ask provider questions in the private sector, which may simply reflect customer accountability as opposed to differential patient sorting.

Even though the mean differences in patient characteristics across sectors are small and largely insignificant, one might still worry about differences in distributions. For our public- 
private comparisons to be valid, what we need is that for any given set of symptoms and conditions, there are enough patients who visit the private sector and those who visit the public, i.e. a common support assumption.

To show that there is common support, we construct propensity scores of visiting a public or a private provider for a given set of symptoms and conditions and plot the empirical distributions of the propensity scores (Figure A.5). The x-axis in Figure A.5 is the predicted probability of visiting a public or private provider which we obtain by regressing a private dummy on symptoms, patient physical activity, and background and demographic variables (rows in Table A.14). The y-axis is the density of each propensity score estimate, by sector. The figure shows that for a given probability of visiting a public or private provider (which represents the same set of symptoms, activity and patient background), there are providers of both types - public and private - throughout the probability distribution. These results suggest that differential patient sorting is not likely to affect our results. 


\section{Appendix Figures and Tables}

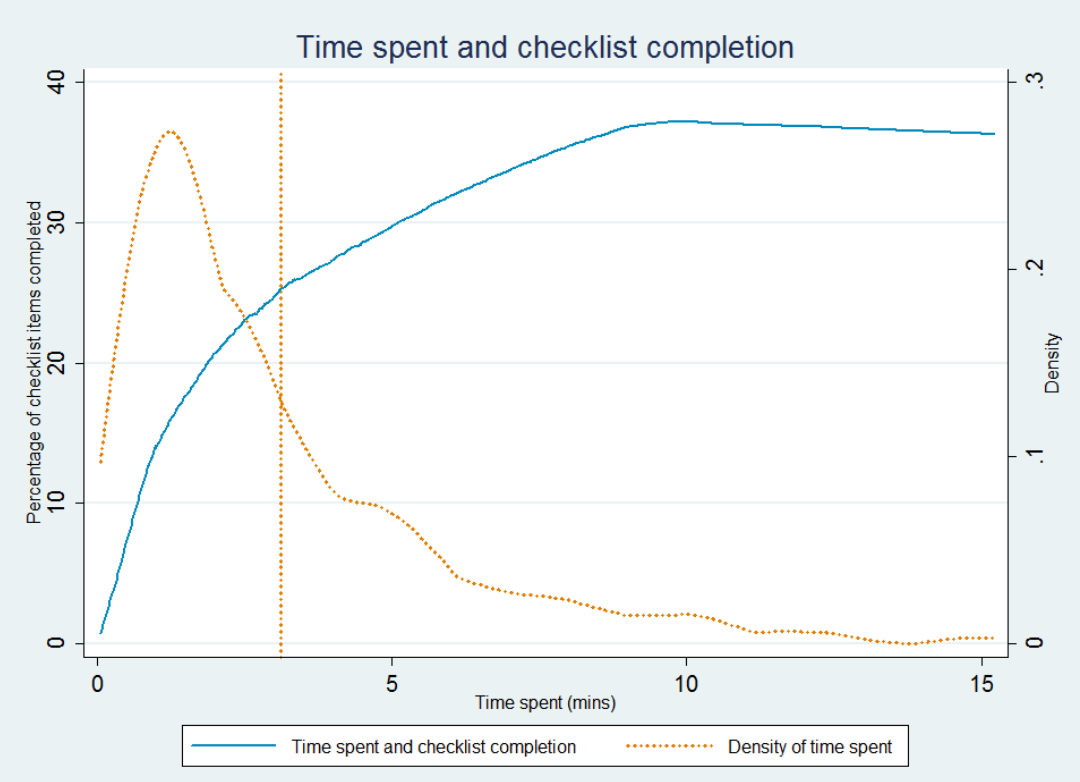

$\ddot{\infty}$

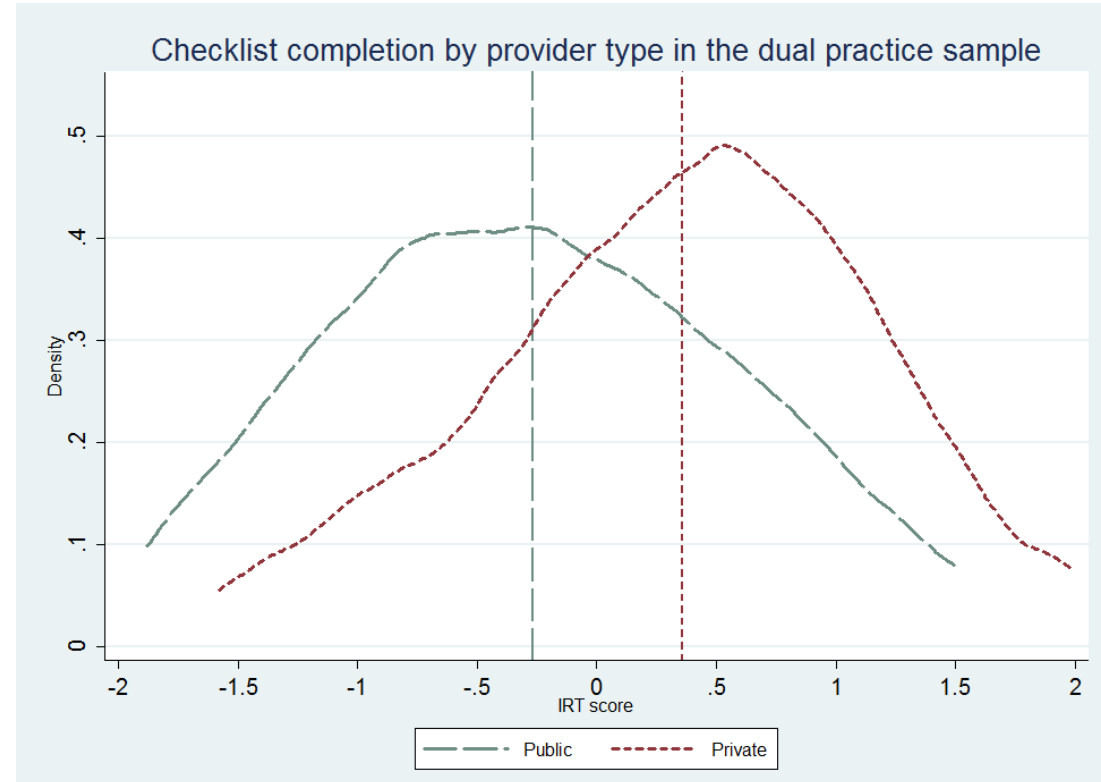

Figure A.3

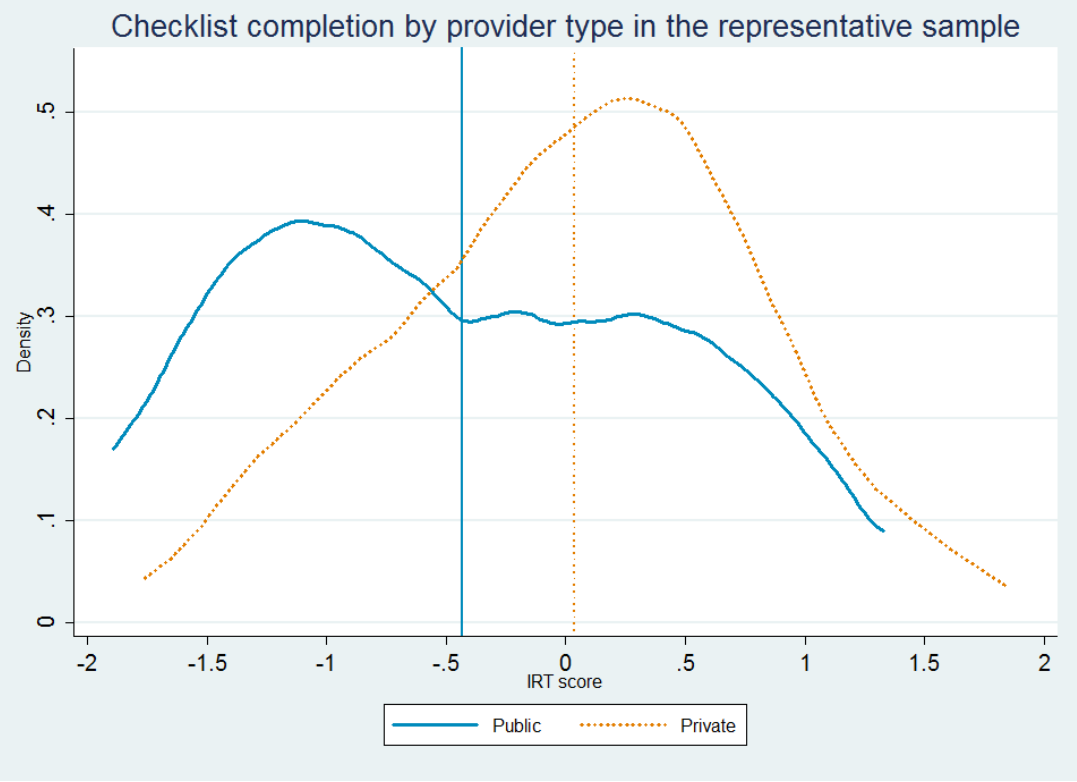

Figure A.2

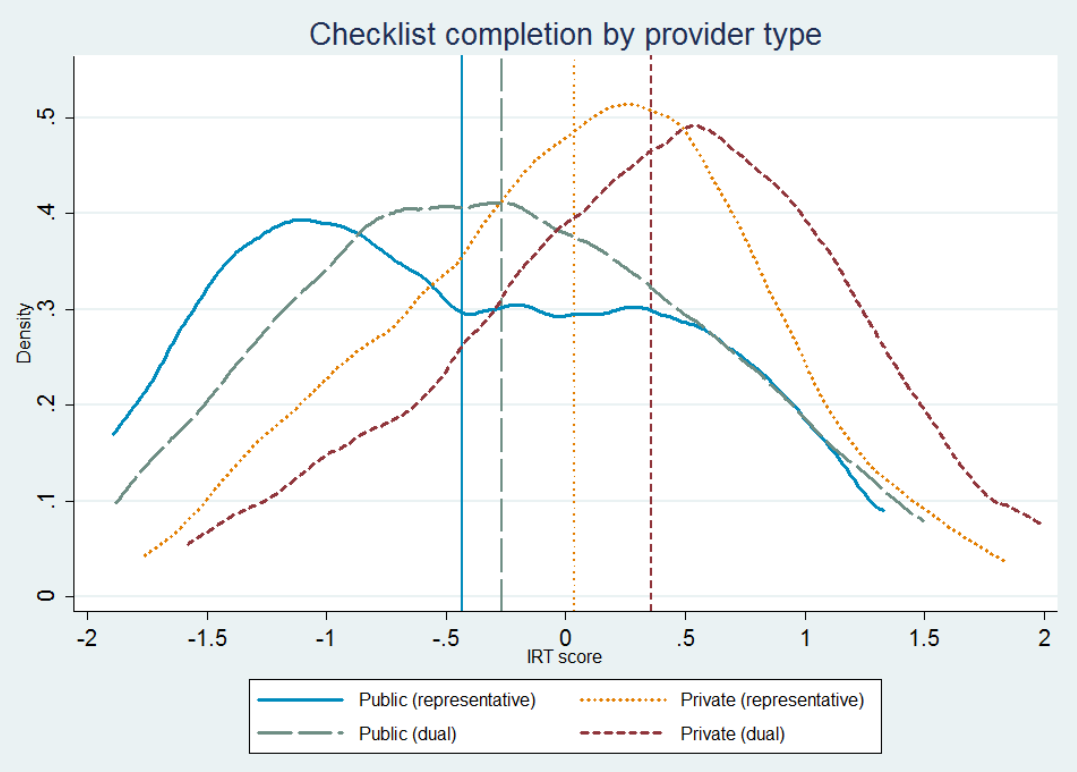

Figure A.4 


\section{Real patients' sorting across sectors}

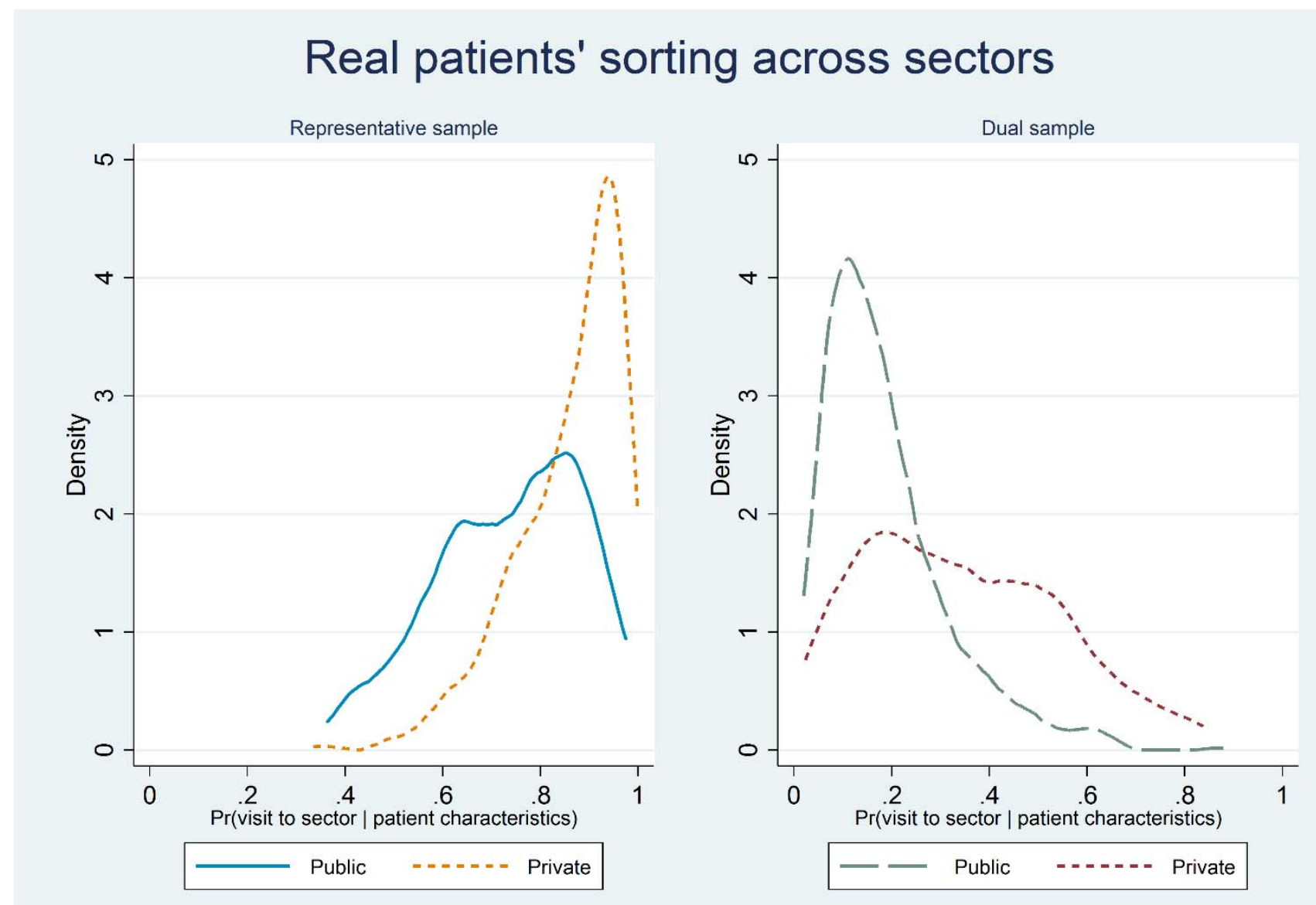

Notes: Patients' characteristics include symptoms presenting, activities of daily living, and patient demographics

Figure A.5 
Table A.1: Sampling and completion of SPs in the representative sample

\begin{tabular}{|c|c|c|c|c|c|c|c|}
\hline & (1) & (2) & (3) & (4) & (5) & (6) & (7) \\
\hline & \multirow{2}{*}{ Markets } & \multicolumn{3}{|c|}{ Number of providers } & \multicolumn{3}{|c|}{ Number of MBBS providers } \\
\hline & & Total & Public & Private & Total & Public & Private \\
\hline \multicolumn{8}{|l|}{ Panel A: Sampling and completion by market } \\
\hline Total eligible & 60 & 719 & 144 & 575 & 51 & 23 & 28 \\
\hline Markets selected for SP & 46 & 649 & 130 & 519 & 50 & 23 & 27 \\
\hline \multicolumn{8}{|l|}{ Reasons for not sampling market } \\
\hline No eligible provider & 7 & & & & & & \\
\hline Common cluster market, no provider within village & 2 & & & & & & \\
\hline Sampled for SPs & & 247 & 45 & 202 & 28 & 12 & 16 \\
\hline Not sampled for SPs & 14 & 472 & 99 & 373 & 23 & 11 & 12 \\
\hline Completed SPs & 46 & 224 & 36 & 188 & 23 & 9 & 14 \\
\hline \multicolumn{8}{|l|}{ Panel B: Sampling and completion by sector } \\
\hline At least 1 public provider sampled & 22 & 151 & 36 & 115 & 20 & 9 & 11 \\
\hline At least 1 public provider completed & 20 & 141 & 36 & 105 & 20 & 9 & 11 \\
\hline At least 1 public MBBS provider sampled & 10 & 98 & 21 & 77 & 18 & 8 & 10 \\
\hline At least 1 public MBBS provider completed & 9 & 87 & 19 & 68 & 18 & 9 & 9 \\
\hline \multicolumn{8}{|l|}{ Private Sector } \\
\hline At least 1 private provider sampled & 44 & 218 & 30 & 188 & 22 & 8 & 14 \\
\hline At least 1 private provider completed & 44 & 218 & 30 & 188 & 22 & 8 & 14 \\
\hline At least 1 private MBBS provider sampled & 8 & 68 & 5 & 63 & 16 & 2 & 14 \\
\hline At least 1 private MBBS provider completed & 7 & 67 & 5 & 62 & 16 & 2 & 14 \\
\hline \multicolumn{8}{|l|}{ Private and Public Sector } \\
\hline Markets with at least 1 public and 1 private provider sampled & 20 & 145 & 30 & 115 & 19 & 8 & 11 \\
\hline Markets with at least 1 public and 1 private provider completed & 18 & 135 & 30 & 105 & 19 & 8 & 11 \\
\hline
\end{tabular}

Notes: In 5 markets where SP work was over completed, the SP saw a provider other than a sampled provider 
Table A.2: Mapping, sampling and completion in the dual practice sample

\begin{tabular}{|c|c|c|c|c|c|}
\hline \multirow[b]{2}{*}{ Facilities } & (1) & (2) & \multirow[b]{2}{*}{ Providers } & (3) & (4) \\
\hline & $\begin{array}{c}\text { Number of } \\
\text { facilities }\end{array}$ & Percentage & & $\begin{array}{c}\text { Number of } \\
\text { providers }\end{array}$ & Percentage \\
\hline \multicolumn{6}{|c|}{ Panel A: Mapping } \\
\hline Total & 200 & & Total & 216 & \\
\hline without doctors & 40 & $20.0 \%$ & without private facilities & 84 & $38.9 \%$ \\
\hline with doctors & 160 & $80.0 \%$ & with private facilities & 132 & $61.1 \%$ \\
\hline \multicolumn{6}{|c|}{ Panel B: Sampling } \\
\hline \multirow[t]{3}{*}{ Total } & 143 & & Total & 143 & \\
\hline & & & without private facilities & 49 & $34.3 \%$ \\
\hline & & & with private facilties & 94 & $65.7 \%$ \\
\hline \multicolumn{6}{|c|}{ Panel C: Completed (at least one case) } \\
\hline \multirow[t]{3}{*}{ Total } & 102 & & Total & 118 & \\
\hline & & & without private facilities & 30 & $25.4 \%$ \\
\hline & & & with private facilities & 88 & $74.6 \%$ \\
\hline
\end{tabular}

Notes: Reasons for not completing SP surveys include transferred and provider not found. In almost all cases our field staff made at least three attempts to complete a case. During fieldwork we replaced five sampled providers with other providers. In two cases, it was because the provider was on sick leave, two cases because provider had been transferred and one case because provider had gone on training. 
Table A.3: Randomization balance for dual sample providers' assignment of Unstable Angina cases

\begin{tabular}{|c|c|c|c|c|c|c|c|c|c|}
\hline & (1) & (2) & (3) & (4) & (5) & (7) & (8) & (9) & (10) \\
\hline & \multicolumn{7}{|c|}{ Asthma outcomes } & \multicolumn{2}{|c|}{ Dysentery outcomes } \\
\hline & $\begin{array}{l}\text { Time spent } \\
\text { (mins) }\end{array}$ & $\begin{array}{c}\text { Percent } \\
\text { checklist } \\
\text { completed }\end{array}$ & $\begin{array}{c}\text { Gave } \\
\text { diagnosis }\end{array}$ & $\begin{array}{l}\text { Correct } \\
\text { diagnosis }\end{array}$ & $\begin{array}{l}\text { Correct } \\
\text { treatment }\end{array}$ & $\begin{array}{l}\text { Palliative } \\
\text { treatment }\end{array}$ & $\begin{array}{c}\text { Unnecessary } \\
\text { treatment }\end{array}$ & $\begin{array}{l}\text { Time spent } \\
\text { (mins) }\end{array}$ & $\begin{array}{c}\text { Percent } \\
\text { checklist } \\
\text { completed }\end{array}$ \\
\hline Is private & $\begin{array}{c}1.497 * * * \\
(0.483)\end{array}$ & $\begin{array}{c}13.190^{* * *} \\
(3.292)\end{array}$ & $\begin{array}{c}0.181 \\
(0.118)\end{array}$ & $\begin{array}{c}0.077 \\
(0.099)\end{array}$ & $\begin{array}{c}0.131 \\
(0.113)\end{array}$ & $\begin{array}{c}-0.230^{* *} \\
(0.117)\end{array}$ & $\begin{array}{l}-0.017 \\
(0.075)\end{array}$ & $\begin{array}{c}0.302 \\
(0.241)\end{array}$ & $\begin{array}{l}9.109^{* *} \\
(4.119)\end{array}$ \\
\hline Received Unstable Angina in private & $\begin{array}{c}0.433 \\
(0.518)\end{array}$ & $\begin{array}{c}5.441 \\
(3.534)\end{array}$ & $\begin{array}{l}0.100 \\
(0.127)\end{array}$ & $\begin{array}{c}0.075 \\
(0.106)\end{array}$ & $\begin{array}{l}-0.194 \\
(0.121)\end{array}$ & $\begin{array}{l}-0.079 \\
(0.126)\end{array}$ & $\begin{array}{c}0.094 \\
(0.080)\end{array}$ & $\begin{array}{c}0.205 \\
(0.255)\end{array}$ & $\begin{array}{l}-0.862 \\
(4.356)\end{array}$ \\
\hline $\begin{array}{l}\text { (Is private) } \mathrm{x} \\
\text { (Received Unstable Angina in private) }\end{array}$ & 0.143 & -2.996 & -0.214 & -0.094 & 0.044 & 0.131 & -0.051 & 0.268 & -0.604 \\
\hline & $(0.719)$ & $(4.898)$ & $(0.176)$ & $(0.147)$ & $(0.168)$ & $(0.174)$ & $(0.111)$ & $(0.354)$ & $(6.053)$ \\
\hline Constant & $\begin{array}{c}1.644^{* * *} \\
(0.347)\end{array}$ & $\begin{array}{c}13.687 * * * \\
(2.367)\end{array}$ & $\begin{array}{c}0.307^{* * *} \\
(0.085)\end{array}$ & $\begin{array}{c}0.150^{* *} \\
(0.071)\end{array}$ & $\begin{array}{c}0.639 * * * \\
(0.081)\end{array}$ & $\begin{array}{c}0.487^{* * *} \\
(0.084)\end{array}$ & $\begin{array}{c}0.873 * * * \\
(0.054)\end{array}$ & $\begin{array}{c}0.783^{* * *} \\
(0.172)\end{array}$ & $\begin{array}{c}17.088^{* * *} \\
(2.941)\end{array}$ \\
\hline
\end{tabular}

Notes: *** Significant at $1 \%, * *$ Significant at $5 \%, *$ Significant at $10 \%$. Robust standard errors are in parenthesis. All regressions include district fixed effects. 
Table A.4: Checklist items, diagnoses and treatments

\begin{tabular}{|c|c|c|c|}
\hline & $\begin{array}{c}\text { (1) } \\
\text { Unstable angina }\end{array}$ & $\begin{array}{c}\text { (2) } \\
\text { Asthma }\end{array}$ & $\begin{array}{c}\text { (3) } \\
\text { Dysentery }\end{array}$ \\
\hline \multicolumn{4}{|c|}{ Panel A: Checklist Items } \\
\hline History questions & $\begin{array}{l}\text { where is the pain, when started, } \\
\text { severity of pain, radiation, previous } \\
\text { similar, since when, shortness of } \\
\text { breath, sw eating, beedi-cigarette, } \\
\text { family history }\end{array}$ & $\begin{array}{l}\text { current breathing probes, cough, } \\
\text { expectoration probes, previous } \\
\text { breathing problems, since when } \\
\text { problems, shortness constant of } \\
\text { episodic, what triggers, fever, chest } \\
\text { pain, weight loss, beedi-cigarette, } \\
\text { family history }\end{array}$ & $\begin{array}{l}\text { age of child, qualities of school, } \\
\text { frequency, quantity of stool, } \\
\text { urination, child active/ playful, fever, } \\
\text { abdominal pain, vomitting, source } \\
\text { of water, what has child eaten, child } \\
\text { taking fluids }\end{array}$ \\
\hline Examinations & $\begin{array}{l}\text { pulse, bp, auscultation (front or } \\
\text { back), temperature attempt, ecg } \\
\text { in/outside clinic }\end{array}$ & $\begin{array}{l}\text { pulse, bp, auscultation (front or } \\
\text { back), temperature attempt }\end{array}$ & \\
\hline \multicolumn{4}{|l|}{ Panel B: Diagnosis } \\
\hline Correct & $\begin{array}{l}\text { Heart attack, angina, myocardial } \\
\text { infarction, attack }\end{array}$ & Asthma, asthma attack & Dysentery, bacteria \\
\hline Incorrect & $\begin{array}{l}\text { Blood pressure problem, } \\
\text { gastrointestinal problem, muscle } \\
\text { problem, the weather, injury, nerve } \\
\text { pull, lack of blood, swelling in chest, } \\
\text { pain from drinking cold water, } \\
\text { heavy work, bad blood, decaying } \\
\text { lungs, chest congestion }\end{array}$ & $\begin{array}{l}\text { Blood pressure problem, } \\
\text { gastrointensinal problem, heart } \\
\text { problem, the weather, cough in } \\
\text { chest, thyroid problem, weakness, } \\
\text { lack of blood, infection in windpipe, } \\
\text { pregnancy, allergy }\end{array}$ & $\begin{array}{l}\text { Weather, heat in liver, acidity, } \\
\text { diarrhea }\end{array}$ \\
\hline \multicolumn{4}{|l|}{ Panel C: Treatment } \\
\hline Correct & $\begin{array}{l}\text { Aspirin, clopidogrel/other anti- } \\
\text { platelet agents, do an ECG. }\end{array}$ & $\begin{array}{l}\text { Bronchodilators, theophylline, } \\
\text { inhaled or oral corticosteroids, } \\
\text { leukotriene inhibitors, cromones, } \\
\text { inhaled anticholinergics }\end{array}$ & ORS, rehydration \\
\hline Palliative & $\begin{array}{l}\text { Nitroglycerin, blood thinners, } \\
\text { betablockers, ACE inhibitors, } \\
\text { vasodilators, other cardiac } \\
\text { medication, morphine, other pain } \\
\text { medication, referral or referral for } \\
\text { an ECG. }\end{array}$ & Anti-allergy medication & Antibiotics,zinc \\
\hline Unncessary or harmful & $\begin{array}{l}\text { Antibiotics, oral rehydration salts, } \\
\text { oral electrolyte solution, zinc, } \\
\text { steroids, inhaler, bronchodilators, } \\
\text { theophylline, inhaled corticosteroids, } \\
\text { leukotriene inhibitors, cromones, } \\
\text { inhaled anti-cholinergics, oral cortico- } \\
\text { steroids, other anti-asthmatic } \\
\text { medication, anti-allergy medication, } \\
\text { psychiatric medication. }\end{array}$ & $\begin{array}{l}\text { Aspirin, clopidogrel, anti-platelet } \\
\text { agents, blood thinners, betablockers, } \\
\text { ACE inhibitors, vasodilators, other } \\
\text { cardiac medication, morphine, other } \\
\text { pain medication, oral rehydration } \\
\text { - salts, oral electrolyte solution, zinc, } \\
\text { antibiotics, anti-ulcer medication, } \\
\text { psychiatric medication }\end{array}$ & $\begin{array}{l}\text { Aspirin, clopidogrel, anti-platelet } \\
\text { agents, blood thinners, betablockers, } \\
\text { ACE inhibitors, vasodilators, other } \\
\text { cardiac medication, morphine, other } \\
\text { pain medication, steroids, inhaler, } \\
\text { bronchodilators, theophylline, } \\
\text { inhaled corticosteroids, leukotriene } \\
\text { inhibitors, cromones, inhaled anti- } \\
\text { cholinergics, oral cortico-steroids, } \\
\text { other anti-asthmatic medication, anti- } \\
\text { allergy medication, psychiatric } \\
\text { medication }\end{array}$ \\
\hline
\end{tabular}

Notes: See Appendix B for coding of treatments 
Table A.5: List of checklist items used in the treatment of SPs

\begin{tabular}{|c|c|c|c|c|c|c|c|c|c|}
\hline & \multirow{3}{*}{$\begin{array}{c}1) \\
\text { Item } \\
\text { discriminat } \\
\text { ion tercile } \\
\end{array}$} & \multirow{3}{*}{$\begin{array}{l}\text { (2) } \\
\text { All }\end{array}$} & (3) & (4) & (5) & \multirow[t]{2}{*}{ (6) } & (7) & (8) & (9) \\
\hline & & & \multicolumn{3}{|c|}{ Representative sample } & & \multicolumn{3}{|c|}{ Dual practice sample } \\
\hline & & & Public & Private & $\begin{array}{c}\text { Difference } \\
(4)-(3)\end{array}$ & All & Public & Private & $\begin{array}{c}\text { Difference } \\
(9)-(8)\end{array}$ \\
\hline \multicolumn{10}{|l|}{ Panel A: Unstable Angina } \\
\hline \multicolumn{10}{|l|}{ History questions } \\
\hline where is the pain & high & 0.659 & 0.486 & 0.694 & $0.208^{* * *}$ & 0.582 & 0.528 & 0.645 & 0.117 \\
\hline when started & low & 0.369 & 0.270 & 0.389 & $0.119^{*}$ & 0.149 & 0.167 & 0.129 & -0.038 \\
\hline doing when began & high & 0.074 & 0.054 & 0.078 & 0.024 & 0.119 & 0.083 & 0.161 & 0.078 \\
\hline severity of pain & low & 0.258 & 0.162 & 0.278 & $0.116^{*}$ & 0.284 & 0.167 & 0.419 & $0.253^{* *}$ \\
\hline radiation & high & 0.143 & 0.108 & 0.150 & 0.042 & 0.299 & 0.222 & 0.387 & $0.165^{*}$ \\
\hline previous similar & medium & 0.392 & 0.270 & 0.417 & $0.146^{* *}$ & 0.328 & 0.278 & 0.387 & 0.109 \\
\hline since when & low & 0.263 & 0.216 & 0.272 & 0.056 & 0.209 & 0.111 & 0.323 & $0.211^{* *}$ \\
\hline quality of pain & high & 0.115 & 0.108 & 0.117 & 0.009 & 0.179 & 0.111 & 0.258 & $0.147^{*}$ \\
\hline pain changes & low & 0.060 & 0.054 & 0.061 & 0.007 & 0.104 & 0.056 & 0.161 & $0.106^{*}$ \\
\hline shortness of breath & medium & 0.138 & 0.081 & 0.150 & 0.069 & 0.045 & 0.056 & 0.032 & -0.023 \\
\hline nausea & medium & 0.295 & 0.297 & 0.294 & -0.003 & 0.209 & 0.056 & 0.387 & $0.332 * * *$ \\
\hline sweating & high & 0.290 & 0.270 & 0.294 & 0.024 & 0.313 & 0.194 & 0.452 & $0.257^{* *}$ \\
\hline beedi-cigarette & low & 0.069 & 0.054 & 0.072 & 0.018 & 0.134 & 0.083 & 0.194 & $0.110^{*}$ \\
\hline family history & high & 0.014 & 0.000 & 0.017 & 0.017 & 0.045 & 0.000 & 0.097 & $0.097^{* *}$ \\
\hline \multicolumn{10}{|l|}{ Examination questions } \\
\hline pulse & low & 0.392 & 0.243 & 0.422 & $0.179 * *$ & 0.537 & 0.417 & 0.677 & $0.261 * *$ \\
\hline $\mathrm{bp}$ & medium & 0.313 & 0.135 & 0.350 & $0.215^{* * *}$ & 0.373 & 0.222 & 0.548 & $0.326^{* * *}$ \\
\hline auscultation (either front or back) & low & 0.447 & 0.189 & 0.500 & $0.311^{* * *}$ & 0.522 & 0.444 & 0.613 & $0.168^{*}$ \\
\hline temperature attempt & medium & 0.134 & 0.108 & 0.139 & 0.031 & 0.134 & 0.028 & 0.258 & $0.230^{* * *}$ \\
\hline ecg in/outside clinic & medium & 0.230 & 0.243 & 0.228 & -0.015 & 0.313 & 0.278 & 0.355 & 0.077 \\
\hline Number of observations & & 217 & 37 & 180 & & 67 & 36 & 31 & \\
\hline \multicolumn{10}{|l|}{ Panel B: Asthma } \\
\hline \multicolumn{10}{|l|}{ History questions } \\
\hline current breathing probes & medium & 0.601 & 0.385 & 0.647 & $0.262^{* * *}$ & 0.552 & 0.422 & 0.671 & $0.250 * * *$ \\
\hline cough & low & 0.677 & 0.590 & 0.696 & 0.106 & 0.575 & 0.453 & 0.686 & $0.233^{* * *}$ \\
\hline expectoration probes & low & 0.148 & 0.077 & 0.163 & $0.086^{*}$ & 0.045 & 0.016 & 0.071 & $0.056^{*}$ \\
\hline previous breathing problems & high & 0.439 & 0.333 & 0.462 & $0.129 *$ & 0.410 & 0.266 & 0.543 & $0.277^{* * *}$ \\
\hline previous episode probes & medium & 0.184 & 0.128 & 0.196 & 0.067 & 0.201 & 0.109 & 0.286 & $0.176^{* * *}$ \\
\hline since when problems & medium & 0.475 & 0.385 & 0.495 & 0.110 & 0.328 & 0.234 & 0.414 & $0.180^{* *}$ \\
\hline how often happens & high & 0.108 & 0.128 & 0.103 & -0.025 & 0.067 & 0.047 & 0.086 & 0.039 \\
\hline shortness constant or episodic & low & 0.103 & 0.051 & 0.114 & 0.063 & 0.090 & 0.047 & 0.129 & $0.082^{* *}$ \\
\hline what triggers & medium & 0.117 & 0.077 & 0.125 & 0.048 & 0.164 & 0.094 & 0.229 & $0.135^{* *}$ \\
\hline how long lasts & high & 0.067 & 0.077 & 0.065 & -0.012 & 0.052 & 0.016 & 0.086 & $0.070^{* *}$ \\
\hline childhood illness & medium & 0.027 & 0.000 & 0.033 & 0.033 & 0.030 & 0.016 & 0.043 & 0.027 \\
\hline age & high & 0.170 & 0.308 & 0.141 & $-0.166^{* * *}$ & 0.537 & 0.578 & 0.500 & -0.078 \\
\hline fever & low & 0.309 & 0.231 & 0.326 & 0.095 & 0.306 & 0.219 & 0.386 & $0.167^{* *}$ \\
\hline chest pain & low & 0.336 & 0.154 & 0.375 & $0.221 * * *$ & 0.231 & 0.172 & 0.286 & $0.114^{*}$ \\
\hline w eight loss & high & 0.000 & 0.000 & 0.000 & 0.000 & 0.015 & 0.016 & 0.014 & -0.001 \\
\hline night sweats & high & 0.054 & 0.051 & 0.054 & 0.003 & 0.067 & 0.047 & 0.086 & 0.039 \\
\hline beedi-cigarette & high & 0.018 & 0.026 & 0.016 & -0.009 & 0.045 & 0.016 & 0.071 & $0.056^{*}$ \\
\hline family history & medium & 0.022 & 0.000 & 0.027 & 0.027 & 0.037 & 0.031 & 0.043 & 0.012 \\
\hline \multicolumn{10}{|l|}{ Examination questions } \\
\hline pulse & low & 0.502 & 0.256 & 0.554 & $0.298^{* * *}$ & 0.388 & 0.313 & 0.457 & $0.145^{* *}$ \\
\hline bp & medium & 0.278 & 0.205 & 0.293 & 0.088 & 0.239 & 0.109 & 0.357 & $0.248^{* * *}$ \\
\hline auscultation (either front or back) & low & 0.516 & 0.333 & 0.554 & $0.221^{* * *}$ & 0.649 & 0.484 & 0.800 & $0.316^{* * *}$ \\
\hline temp attempt & low & 0.166 & 0.103 & 0.179 & 0.077 & 0.082 & 0.063 & 0.100 & 0.038 \\
\hline Number of observations & & 223 & 39 & 184 & & 134 & 64 & 70 & \\
\hline
\end{tabular}


Table A.5 continued

\begin{tabular}{|c|c|c|c|c|c|c|c|c|c|}
\hline & \multirow{3}{*}{$\begin{array}{c}(1) \\
\text { Item } \\
\text { discriminat } \\
\text { ion tercile }\end{array}$} & & (2) & (3) & (4) & & $(5)$ & (6) & (7) \\
\hline & & \multicolumn{4}{|c|}{ Representative sample } & \multicolumn{4}{|c|}{ Dual practice sample } \\
\hline & & All & Public & Private & $\begin{array}{c}\text { Difference } \\
(3)-(2)\end{array}$ & All & Public & Private & $\begin{array}{c}\text { Difference } \\
(6)-(5)\end{array}$ \\
\hline \multicolumn{10}{|l|}{ Panel C: Dysentery } \\
\hline \multicolumn{10}{|l|}{ History questions } \\
\hline age of child & low & 0.919 & 0.795 & 0.945 & $0.150^{* * *}$ & 0.930 & 0.921 & 0.939 & 0.019 \\
\hline qualities of stool & low & 0.167 & 0.077 & 0.186 & $0.109^{* *}$ & 0.271 & 0.159 & 0.379 & $0.220^{* * *}$ \\
\hline frequency & medium & 0.288 & 0.179 & 0.311 & $0.132^{* *}$ & 0.372 & 0.270 & 0.470 & $0.200^{* * *}$ \\
\hline quantity of stool & high & 0.050 & 0.000 & 0.060 & $0.060^{*}$ & 0.031 & 0.016 & 0.045 & 0.030 \\
\hline urination & high & 0.018 & 0.000 & 0.022 & 0.022 & 0.008 & 0.016 & 0.000 & -0.016 \\
\hline active/playful & high & 0.032 & 0.026 & 0.033 & 0.007 & 0.000 & 0.000 & 0.000 & 0.000 \\
\hline fever & medium & 0.171 & 0.077 & 0.191 & $0.114 * *$ & 0.295 & 0.222 & 0.364 & $0.141^{* *}$ \\
\hline abdominal pain & low & 0.113 & 0.077 & 0.120 & 0.043 & 0.256 & 0.222 & 0.288 & 0.066 \\
\hline vomiting & low & 0.216 & 0.077 & 0.246 & $0.169 * * *$ & 0.295 & 0.254 & 0.333 & 0.079 \\
\hline source of water & high & 0.023 & 0.000 & 0.027 & 0.027 & 0.016 & 0.000 & 0.030 & $0.030^{*}$ \\
\hline what has eaten & medium & 0.050 & 0.000 & 0.060 & $0.060^{*}$ & 0.093 & 0.032 & 0.152 & $0.120^{* * *}$ \\
\hline taking fluids & medium & 0.023 & 0.000 & 0.027 & 0.027 & 0.062 & 0.048 & 0.076 & 0.028 \\
\hline Number of observations & & 222 & 39 & 183 & & 130 & 63 & 67 & \\
\hline
\end{tabular}


Table A.6: Effort in the public and private sectors by checklist item discrimination terciles
(1)
(2)
(3)
(4)
(5)
(6)

Outcome variable: Percentage of recommended type of checklist items

Representative sample

Dual practice sample

Low Medium High

Low Medium High

discrimination discrimination discrimination discrimination discrimination discrimination

\begin{tabular}{|c|c|c|c|c|c|c|}
\hline \multicolumn{7}{|c|}{ Panel A: SP and case fixed effects } \\
\hline \multirow[t]{2}{*}{ Is a private provider } & $10.982^{* * *}$ & $7.085^{* *}$ & 1.760 & $10.519 * * *$ & $11.745^{* * *}$ & $5.122^{* * *}$ \\
\hline & $(3.281)$ & $(2.875)$ & $(2.143)$ & $(2.404)$ & $(2.360)$ & $(1.746)$ \\
\hline R-squared & 0.144 & 0.175 & 0.238 & 0.279 & 0.236 & 0.318 \\
\hline Number of observations & 662 & 662 & 662 & 330 & 330 & 330 \\
\hline Mean of public & 21.770 & 13.975 & 10.197 & 28.254 & 14.592 & 10.020 \\
\hline Mean of private & 32.966 & 21.322 & 12.235 & 41.104 & 28.800 & 15.234 \\
\hline Mean of sample & 32.108 & 20.759 & 12.079 & 34.756 & 21.782 & 12.659 \\
\hline \multicolumn{7}{|c|}{ Panel B: SP, case and market/district fixed effects } \\
\hline \multirow{2}{*}{ Is a private provider } & $11.290^{* * *}$ & $8.597 * * *$ & 1.594 & $10.594 * * *$ & $11.709 * * *$ & $5.077 * * *$ \\
\hline & $(2.609)$ & $(2.535)$ & $(1.969)$ & $(2.358)$ & $(2.381)$ & $(1.751)$ \\
\hline R-squared & 0.253 & 0.256 & 0.300 & 0.301 & 0.247 & 0.322 \\
\hline Number of observations & 662 & 662 & 662 & 330 & 330 & 330 \\
\hline \multicolumn{7}{|c|}{ Panel C: SP, case and market/district fixed effects } \\
\hline \multirow[t]{2}{*}{ Is a private provider } & $8.538^{* * *}$ & $7.317^{* *}$ & 1.657 & $11.786^{* * *}$ & $12.518^{* * *}$ & $4.523 * *$ \\
\hline & $(3.030)$ & $(3.092)$ & $(2.381)$ & $(2.480)$ & $(2.466)$ & $(1.794)$ \\
\hline \multirow[t]{2}{*}{ Has MBBS } & 2.548 & 5.175 & 2.307 & & & \\
\hline & $(3.949)$ & $(3.449)$ & $(2.916)$ & & & \\
\hline \multirow[t]{2}{*}{ Has some qualification } & 2.300 & $4.764^{*}$ & 0.721 & & & \\
\hline & $(2.563)$ & $(2.447)$ & $(1.831)$ & & & \\
\hline \multirow[t]{2}{*}{ Age of provider } & -0.151 & -0.009 & 0.044 & -0.069 & -0.135 & -0.042 \\
\hline & $(0.102)$ & $(0.094)$ & $(0.077)$ & $(0.139)$ & $(0.138)$ & $(0.101)$ \\
\hline \multirow[t]{2}{*}{ Gender of provider (1=Male) } & 1.009 & -1.353 & -2.369 & 2.773 & -2.792 & -3.651 \\
\hline & $(4.541)$ & $(5.383)$ & $(3.361)$ & $(3.586)$ & $(3.565)$ & $(2.593)$ \\
\hline \multirow[t]{2}{*}{ Patient load during visit } & -0.041 & -0.396 & 0.050 & -0.501 & -0.203 & -0.211 \\
\hline & $(0.736)$ & $(0.557)$ & $(0.528)$ & $(0.576)$ & $(0.572)$ & $(0.416)$ \\
\hline R-squared & 0.254 & 0.262 & 0.301 & 0.290 & 0.252 & 0.330 \\
\hline Number of observations & 638 & 638 & 638 & 301 & 301 & 301 \\
\hline
\end{tabular}

Notes: *** Significant at $1 \%, * *$ Significant at $5 \%, *$ Significant at $10 \%$. Robust standard errors clustered at the market level are in parenthesis. Observations are standardized provider-patient interactions. Checklist item discrimination parameters are estimated using the IRT methodology. The classification of items into terciles of difficulty is done within each case, but the results are robust to classifying the items jointly across all cases. Market fixed effects are used for the representative sample, and district fixed effects for dual practice sample. 
Table A.7: Effort, diagnosis and treatment by case

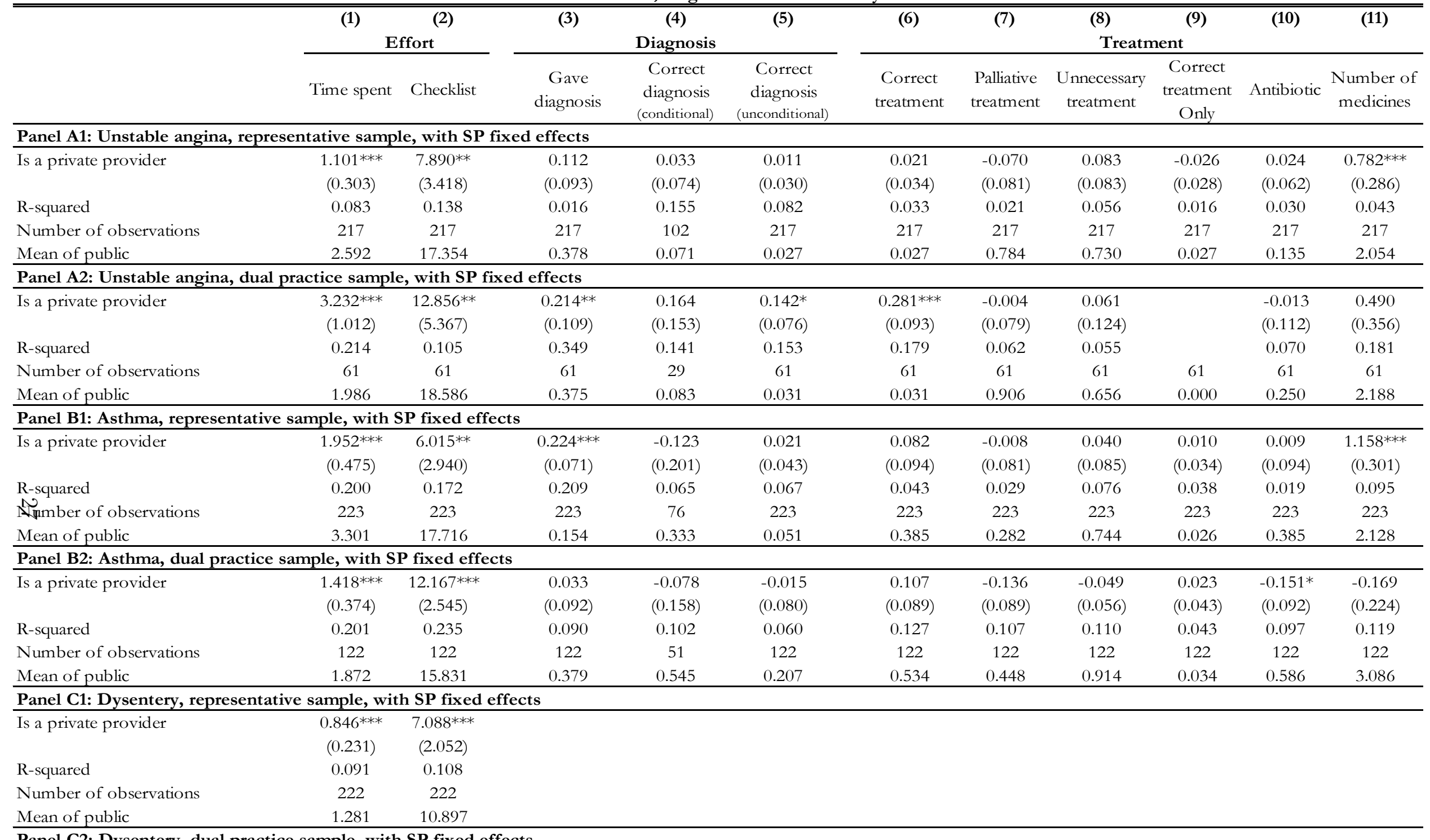

Panel C2: Dysentery, dual practice sample, with SP fixed effects

\begin{tabular}{lcc}
\hline Is a private provider & $0.395^{* *}$ & $5.279^{* *}$ \\
& $(0.181)$ & $(2.569)$ \\
R-squared & 0.095 & 0.340 \\
Number of observations & 119 & 119 \\
Mean of public & 0.879 & 16.228 \\
\hline \hline
\end{tabular}

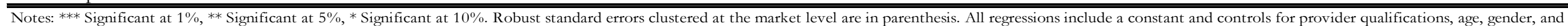
patient load. Observations are standardized provider-patient interactions. In column (11) the dependent variable is total number of medicines recommended to the patient (dispensed and/or prescribed). 
Table A.8: Summary of treatment by case

\begin{tabular}{|c|c|c|c|c|c|c|}
\hline & (1) & (2) & (3) & (4) & (5) & (6) \\
\hline & \multicolumn{3}{|c|}{ Representative sample } & \multicolumn{3}{|c|}{ Dual practice sample } \\
\hline & Public & Private & $\begin{array}{c}\text { Difference } \\
(2)-(1)\end{array}$ & Public & Private & $\begin{array}{c}\text { Difference } \\
(5)-(4)\end{array}$ \\
\hline \multicolumn{7}{|l|}{ Panel A: Unstable Angina } \\
\hline Correct treatment & 0.03 & 0.06 & 0.03 & 0.03 & 0.29 & $0.26 * * *$ \\
\hline Correct treatment (alternate) & 0.46 & 0.37 & -0.09 & 0.42 & 0.61 & $0.20^{*}$ \\
\hline Palliative treatment & 0.78 & 0.71 & -0.07 & 0.92 & 0.90 & -0.01 \\
\hline Unnecessary treatment & 0.73 & 0.80 & 0.07 & 0.67 & 0.74 & 0.08 \\
\hline Aspirin & 0.03 & 0.04 & 0.02 & 0.03 & 0.23 & $0.20^{* * *}$ \\
\hline Anti-platelet agents & 0.03 & 0.01 & -0.02 & 0.00 & 0.03 & 0.03 \\
\hline Referred & 0.30 & 0.24 & -0.05 & 0.22 & 0.32 & 0.10 \\
\hline ECG & 0.24 & 0.23 & -0.02 & 0.28 & 0.35 & 0.08 \\
\hline ECG \& Referred & 0.11 & 0.12 & 0.01 & 0.08 & 0.16 & 0.08 \\
\hline Antibiotic & 0.14 & 0.17 & 0.03 & 0.28 & 0.23 & -0.05 \\
\hline Number of observations & 37 & 180 & & 36 & 31 & \\
\hline \multicolumn{7}{|l|}{ Panel B: Asthma } \\
\hline Correct treatment & 0.38 & 0.50 & $0.12^{*}$ & 0.58 & 0.67 & 0.09 \\
\hline Palliative treatment & 0.28 & 0.29 & 0.01 & 0.47 & 0.29 & $-0.18^{* *}$ \\
\hline Unnecessary treatment & 0.74 & 0.83 & $0.09 *$ & 0.92 & 0.89 & -0.04 \\
\hline Bronchodilators & 0.33 & 0.36 & 0.03 & 0.52 & 0.59 & 0.07 \\
\hline Theophylline & 0.13 & 0.22 & $0.09 *$ & 0.31 & 0.31 & 0.00 \\
\hline Oral Corticosteroids & 0.15 & 0.31 & $0.16^{* *}$ & 0.16 & 0.24 & 0.09 \\
\hline Antibiotic & 0.38 & 0.40 & 0.02 & 0.59 & 0.46 & $-0.14^{*}$ \\
\hline Number of observations & 39 & 184 & & 64 & 70 & \\
\hline \multicolumn{7}{|l|}{ Panel C: Dysentery } \\
\hline Correct treatment & 0.08 & 0.13 & 0.05 & 0.33 & 0.22 & $-0.11 *$ \\
\hline Palliative treatment & 0.44 & 0.61 & $0.18^{* *}$ & 0.75 & 0.61 & $-0.13^{*}$ \\
\hline Unnecessary treatment & 0.28 & 0.56 & $0.28^{* * *}$ & 0.35 & 0.40 & 0.05 \\
\hline ORS & 0.05 & 0.12 & 0.07 & 0.33 & 0.21 & $-0.12^{*}$ \\
\hline Asked to see child & 0.33 & 0.14 & $-0.20 * * *$ & 0.27 & 0.42 & $0.15^{* *}$ \\
\hline Antibiotic & 0.44 & 0.61 & $0.18^{* *}$ & 0.75 & 0.61 & $-0.13^{*}$ \\
\hline Number of observations & 39 & 183 & & 63 & 67 & \\
\hline
\end{tabular}

Notes: In Unstable Angina, alternate definition for correct treatment codes referrals and referrals for ECG as correct. In the dysentery case, note the large and significant differences in "asked to see the child" across public and private providers in the representative and dual samples. If we were to assume the same rate of correct treatment by public and private providers in the cases where they "asked to see the child" as in the cases where a treatment was provided, then the differences in correct treatment are no longer significant in either sample. If we carry out a bounding exercise, the differences are still not significant, and the standard errors are too wide for meaningful inference. This is why we exclude the dysentery case in our pooled analysis of treatment across cases. 
Table A.9: Robustness of treatment results with alternative definition for correct treatment for unstable angina

\begin{tabular}{|c|c|c|c|c|}
\hline & (1) & $(2)$ & (3) & (4) \\
\hline & \multicolumn{2}{|c|}{ All (compare with table 4) } & \multicolumn{2}{|c|}{ Unstable angina only (compare with table A8) } \\
\hline & Representative sample & Dual practice sample & Representative sample & Dual sample \\
\hline & Correct treatment & Correct treatment & Correct treatment & Correct treatment \\
\hline \multicolumn{5}{|l|}{ Panel A: SP fixed effects } \\
\hline \multirow[t]{2}{*}{ Is a private provider } & -0.014 & $0.119^{*}$ & -0.112 & $0.206^{*}$ \\
\hline & $(0.063)$ & $(0.069)$ & $(0.088)$ & $(0.121)$ \\
\hline R-squared & 0.075 & 0.086 & 0.092 & 0.070 \\
\hline Number of observations & 440 & 201 & 217 & 67 \\
\hline Mean of public & 0.421 & 0.520 & 0.459 & 0.417 \\
\hline Mean of private & 0.421 & 0.653 & 0.360 & 0.613 \\
\hline Mean of sample & 0.421 & 0.587 & 0.367 & 0.507 \\
\hline \multicolumn{5}{|c|}{ Panel B: SP and market/district fixed effects } \\
\hline \multirow[t]{2}{*}{ Is a private provider } & 0.001 & $0.122 *$ & -0.065 & $0.195^{*}$ \\
\hline & $(0.069)$ & $(0.070)$ & $(0.118)$ & $(0.115)$ \\
\hline R-squared & 0.196 & 0.095 & 0.298 & 0.186 \\
\hline Number of observations & 440 & 201 & 217 & 67 \\
\hline \multicolumn{5}{|c|}{ Panel C: SP and market/district fixed effects } \\
\hline \multirow[t]{2}{*}{ Is a private provider } & -0.009 & $0.126^{*}$ & -0.203 & 0.190 \\
\hline & $(0.070)$ & $(0.075)$ & $(0.141)$ & $(0.122)$ \\
\hline \multirow[t]{2}{*}{ Has MBBS } & $0.340^{* * *}$ & & 0.233 & \\
\hline & $(0.081)$ & & $(0.147)$ & \\
\hline \multirow[t]{2}{*}{ Has some qualification } & $0.164 * * *$ & & 0.139 & \\
\hline & $(0.057)$ & & $(0.095)$ & \\
\hline \multirow[t]{2}{*}{ Age of provider } & 0.000 & -0.004 & 0.002 & -0.006 \\
\hline & $(0.004)$ & $(0.004)$ & $(0.004)$ & $(0.006)$ \\
\hline \multirow[t]{2}{*}{ Gender of provider $(1=$ Male $)$} & 0.256 & 0.007 & $0.334^{* *}$ & -0.165 \\
\hline & $(0.158)$ & $(0.111)$ & $(0.170)$ & $(0.162)$ \\
\hline \multirow[t]{2}{*}{ Patient load during visit } & $-0.030 * * *$ & -0.006 & $-0.022^{* *}$ & -0.034 \\
\hline & $(0.008)$ & $(0.018)$ & $(0.010)$ & $(0.021)$ \\
\hline R-squared & 0.244 & 0.106 & 0.352 & 0.240 \\
\hline Number of observations & 423 & 183 & 208 & 61 \\
\hline
\end{tabular}

Notes: *** Significant at $1 \%, * *$ Significant at $5 \%, *$ Significant at $10 \%$. Robust standard errors clustered at the market level are in parenthesis. All regressions include a constant. Observations are standardized provider-patient interactions. Columns (1) and (2) also include case fixed effects. Market fixed effects are used for the representative sample, and district fixed effects for dual practice sample. Alternative definition for Unstable Angina adds "referral" and "referral for ECG" as correct treatment. 
Table A.10: Robustness of provider effort results to exclusion of dysentery cases

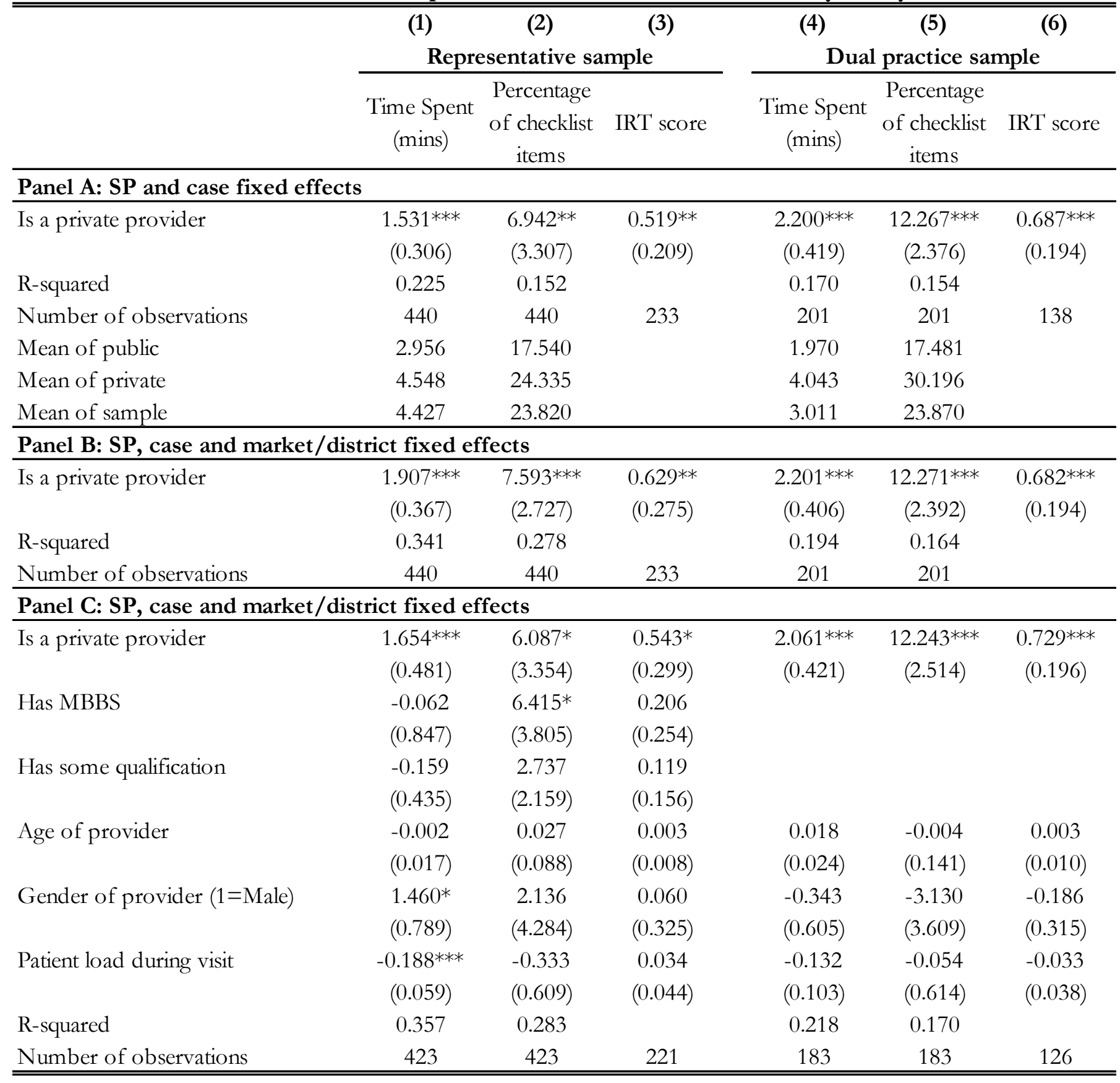

Notes: *** Significant at 1\%, ** Significant at 5\%, * Significant at 10\%. Robust standard errors clustered at the market level are in parenthesis. All regressions include a constant. Observations are standardized provider-patient interactions, except in IRT score column where each observation is a provider. The score is computed using all cases, plausible values scores are used. Market fixed effects are used for the representative sample, and district fixed effects for dual practice sample. 
Table A.11: Robustness of results to inclusion of facilties controls

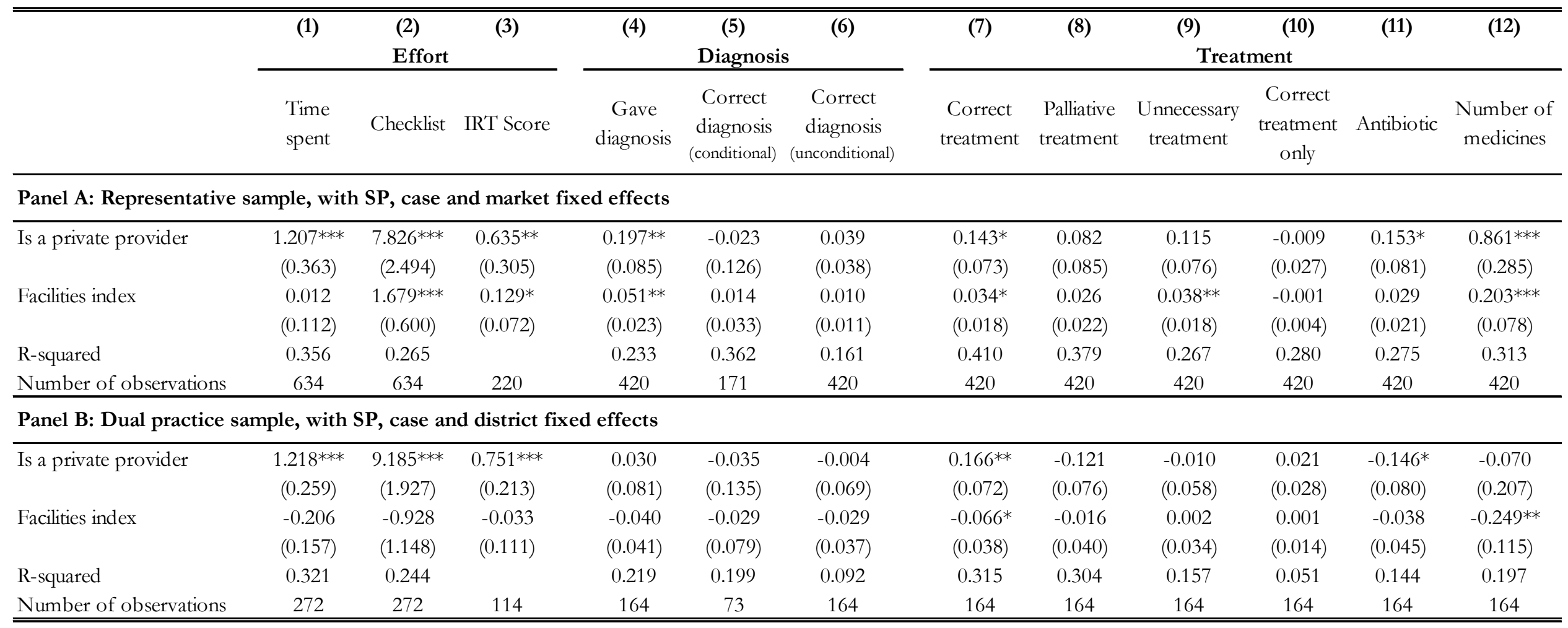

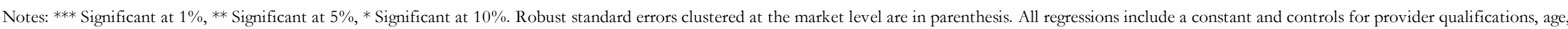

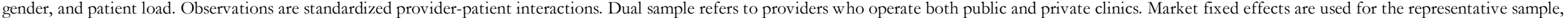

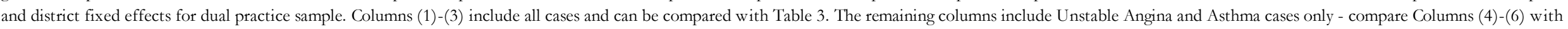

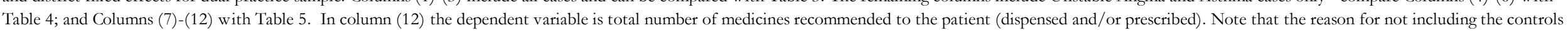

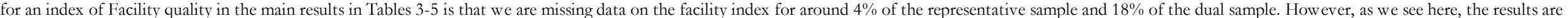
robust to including the facility controls. 
Table A.12: Correlates of price charged

(private interactions, excludes cases where all medicines are unidentifiable)

\begin{tabular}{|c|c|c|c|c|c|c|}
\hline & (1) & (2) & (3) & (4) & (5) & (6) \\
\hline & \multicolumn{6}{|c|}{ Fees in Rs. } \\
\hline & $\begin{array}{c}\text { Binary } \\
\text { regressions }\end{array}$ & $\begin{array}{l}\text { Multiple } \\
\text { regression }\end{array}$ & $\begin{array}{c}\text { Binary } \\
\text { regressions }\end{array}$ & $\begin{array}{l}\text { Multiple } \\
\text { regression }\end{array}$ & $\begin{array}{c}\text { Binary } \\
\text { regressions }\end{array}$ & $\begin{array}{l}\text { Multiple } \\
\text { regression }\end{array}$ \\
\hline \multirow[t]{2}{*}{ Time spent with SP (minutes) } & $1.720^{* * *}$ & 0.618 & $2.625^{* * *}$ & $2.279 * * *$ & $1.484 * * *$ & $0.709 *$ \\
\hline & $(0.476)$ & $(0.477)$ & $(0.587)$ & $(0.692)$ & $(0.377)$ & $(0.401)$ \\
\hline \multirow[t]{2}{*}{ Percentage of checklist items } & $0.397 * * *$ & $0.339 * * *$ & $0.364 * * *$ & 0.055 & $0.386^{* * *}$ & $0.291 * * *$ \\
\hline & $(0.089)$ & $(0.096)$ & $(0.100)$ & $(0.129)$ & $(0.071)$ & $(0.084)$ \\
\hline \multirow[t]{2}{*}{ Correct diagnosis (unconditional) } & -4.269 & $-3.647 *$ & 7.504 & 5.494 & 2.690 & 2.685 \\
\hline & $(3.978)$ & $(1.993)$ & $(9.350)$ & $(9.046)$ & $(4.658)$ & $(4.148)$ \\
\hline \multirow[t]{2}{*}{ Correct treatment } & $6.199 * * *$ & -1.564 & $7.744^{*}$ & 4.475 & $7.306^{* * *}$ & 0.602 \\
\hline & $(1.757)$ & $(2.919)$ & $(4.145)$ & $(4.967)$ & $(1.934)$ & $(2.404)$ \\
\hline \multirow[t]{2}{*}{ Palliative treatment } & $7.711^{* * *}$ & 2.198 & $10.435^{* *}$ & 7.757 & $7.796^{* * *}$ & $3.542 * *$ \\
\hline & $(1.810)$ & $(1.722)$ & $(4.242)$ & $(4.873)$ & $(1.743)$ & $(1.726)$ \\
\hline \multirow[t]{2}{*}{ Unnecessary treatment } & $15.794 * * *$ & 3.147 & $14.973 * * *$ & 5.137 & $15.655^{* * *}$ & $4.888^{*}$ \\
\hline & $(2.842)$ & $(2.963)$ & $(5.032)$ & $(6.240)$ & $(2.451)$ & $(2.746)$ \\
\hline \multirow[t]{2}{*}{ Dispensed medicines } & $19.525^{* * *}$ & $16.400^{* * *}$ & $16.118^{* * *}$ & $12.371 *$ & $16.511 * * *$ & $15.688^{* * *}$ \\
\hline & (2.993) & $(2.726)$ & $(6.070)$ & $(7.019)$ & (2.319) & $(2.830)$ \\
\hline \multirow[t]{2}{*}{ Prescribed medicines } & -2.931 & -4.331 & 7.540 & -2.854 & 0.071 & -4.133 \\
\hline & $(3.600)$ & $(3.639)$ & $(5.997)$ & $(6.734)$ & $(2.918)$ & (3.202) \\
\hline \multirow[t]{2}{*}{ Number of medicines } & $5.540^{* * *}$ & 1.630 & $5.863^{* * *}$ & 3.016 & $5.283^{* * *}$ & 1.111 \\
\hline & $(0.842)$ & (1.394) & $(1.783)$ & $(2.987)$ & $(0.787)$ & $(1.348)$ \\
\hline \multirow[t]{2}{*}{ Referred/Asked to see child } & $-20.348^{* * *}$ & $-10.054 * * *$ & $-9.882^{* *}$ & -4.867 & $-17.533 * * *$ & $-11.860^{* * *}$ \\
\hline & (4.999) & (3.683) & $(4.763)$ & $(4.888)$ & (3.911) & (3.021) \\
\hline \multirow[t]{2}{*}{ Has MBBS } & $23.517 * * *$ & $27.905^{* * *}$ & & & $14.155^{* * *}$ & $23.516^{* * *}$ \\
\hline & $(6.150)$ & $(7.830)$ & & & $(4.369)$ & $(3.923)$ \\
\hline \multirow[t]{2}{*}{ Has some qualification } & 4.305 & $6.067 * * *$ & & & 2.127 & $6.952^{* * *}$ \\
\hline & $(3.768)$ & $(2.282)$ & & & $(3.376)$ & $(2.370)$ \\
\hline \multirow[t]{2}{*}{ Patient load during visit } & 1.017 & $0.867^{* *}$ & -0.073 & -0.285 & 0.512 & 0.276 \\
\hline & $(0.888)$ & $(0.404)$ & $(0.807)$ & $(0.810)$ & $(0.748)$ & $(0.581)$ \\
\hline \multirow[t]{2}{*}{ Age of provider } & -0.186 & -0.111 & 0.267 & 0.248 & -0.119 & -0.018 \\
\hline & $(0.155)$ & $(0.100)$ & $(0.239)$ & $(0.218)$ & $(0.126)$ & $(0.089)$ \\
\hline \multirow[t]{2}{*}{ Gender of provider ( $1=$ Male $)$} & $-8.238^{* *}$ & -5.876 & -1.284 & -3.760 & $-7.475^{* *}$ & -3.810 \\
\hline & (3.518) & $(4.543)$ & $(4.882)$ & $(5.580)$ & $(2.961)$ & $(3.919)$ \\
\hline \multirow[t]{2}{*}{ Constant } & & 9.745 & & -11.295 & & 2.234 \\
\hline & & $(7.179)$ & & $(11.810)$ & & $(6.345)$ \\
\hline $\mathrm{R} 2$ & & 0.446 & & 0.444 & & 0.398 \\
\hline Number of observations & & 495 & & 154 & & 649 \\
\hline Mean price charged & & 27.638 & & 32.740 & & 28.849 \\
\hline $\mathrm{SD}$ & & 26.557 & & 28.592 & & 27.118 \\
\hline
\end{tabular}

Notes: *** Significant at $1 \%$, ** Significant at $5 \%$, * Significant at $10 \%$. Robust standard errors clustered at the market level are in parenthesis. Observations are standardized provider-patient interactions. Interpretation of coefficents in "Binary regressions" needs caution. Each coefficient represents a separate regression of prices on the row variable and case and district fixed effects. Multiple regressions include case and district fixed effects. 
Table A.13: Cost in the public sector

\begin{tabular}{|c|c|c|}
\hline & (1) & $(2)$ \\
\hline Panel A: Staff per facility & $\mathbf{N}$ & Average monthly wage (Rs.) \\
\hline Medical Officer in Charge/Medical Officer & 1.92 & Rs.32,245 \\
\hline GNM/ANM/VHN/LHV & 3.24 & Rs.16,305 \\
\hline MPW/MNA/Assistant/Compounder & 1.43 & Rs.16,657 \\
\hline Pharmacist/Chemist/Lab Assistant/Technician & 0.8 & Rs.16,571 \\
\hline Paramedic/other & 6.08 & Rs.13,387 \\
\hline All & 13.47 & Rs.17,315 \\
\hline Number of facilities & 115 & \\
\hline \multicolumn{3}{|l|}{ Panel B: Visits to the public facilities per month } \\
\hline Year 2008 & 111,039 & \\
\hline Year 2009 & 113,230 & \\
\hline Year 2010 & 111,473 & \\
\hline \multicolumn{3}{|l|}{ Panel C: Average per patient cost } \\
\hline Year 2008 & Rs. 241.87 & \\
\hline Year 2009 & Rs.237.66 & \\
\hline Year 2010 & Rs.241.61 & \\
\hline
\end{tabular}

Notes: We use an extremely conservative measure of per patient cost in the public sector facility. We assume that salary cost are the only cost in running a public health facility. Furthermore, we assume that every patient that visits the public health facility visits for a primary care visit, while people also visit public health facilities for preventative services such as vaccination. Wages data were collected in the year 2010, note that we use 2010 wage data to compute cost per patient in 2008 and 2009. Wages in 2008 and 2009 could have been lower. 
Table A.14: Real patients' characteristics in the public and private sectors

\begin{tabular}{|c|c|c|c|c|c|c|c|c|}
\hline & (1) & (2) & (3) & (4) & (5) & (6) & (7) & (8) \\
\hline & \multicolumn{4}{|c|}{ Representative Sample } & \multicolumn{4}{|c|}{ Dual Practice } \\
\hline & \multirow[t]{2}{*}{ Public } & \multirow[t]{2}{*}{ Private } & \multicolumn{2}{|c|}{$\begin{array}{c}\text { Difference } \\
\text { (coeff. on private) }\end{array}$} & \multirow[t]{2}{*}{ Public } & \multirow[t]{2}{*}{ Private } & \multicolumn{2}{|c|}{$\begin{array}{c}\text { Difference } \\
\text { (coeff. on private) }\end{array}$} \\
\hline & & & $\begin{array}{c}\text { no fixed } \\
\text { effects }\end{array}$ & $\begin{array}{c}\text { market } \\
\text { fixed effects }\end{array}$ & & & $\begin{array}{c}\text { no fixed } \\
\text { effects }\end{array}$ & $\begin{array}{c}\text { market } \\
\text { fixed effects }\end{array}$ \\
\hline \multicolumn{9}{|l|}{ Patient/Case Characteristics } \\
\hline Number of symptoms & 1.446 & 1.568 & $\begin{array}{l}0.122^{* *} \\
(0.057)\end{array}$ & $\begin{array}{c}0.092 \\
(0.081)\end{array}$ & 2.075 & 2.113 & $\begin{array}{c}0.038 \\
(0.095)\end{array}$ & $\begin{array}{c}0.026 \\
(0.101)\end{array}$ \\
\hline Fever & 0.309 & 0.445 & $\begin{array}{c}0.136 \text { *** } \\
(0.034)\end{array}$ & $\begin{array}{c}0.135^{* *} \\
(0.054)\end{array}$ & 0.550 & 0.548 & $\begin{array}{l}-0.002 \\
(0.043)\end{array}$ & $\begin{array}{c}0.012 \\
(0.043)\end{array}$ \\
\hline Cold & 0.272 & 0.195 & $\begin{array}{l}-0.077 \\
(0.049)\end{array}$ & $\begin{array}{l}-0.015 \\
(0.062)\end{array}$ & 0.476 & 0.434 & $\begin{array}{l}-0.042 \\
(0.054)\end{array}$ & $\begin{array}{l}-0.047 \\
(0.050)\end{array}$ \\
\hline Diarrhea & 0.105 & 0.151 & $\begin{array}{c}0.046 \\
(0.033)\end{array}$ & $\begin{array}{c}0.008 \\
(0.040)\end{array}$ & 0.066 & 0.075 & $\begin{array}{c}0.009 \\
(0.014)\end{array}$ & $\begin{array}{c}0.006 \\
(0.015)\end{array}$ \\
\hline Weakness & 0.148 & 0.209 & $\begin{array}{l}0.061 * \\
(0.034)\end{array}$ & $\begin{array}{c}0.047 \\
(0.047)\end{array}$ & 0.182 & 0.176 & $\begin{array}{l}-0.006 \\
(0.029)\end{array}$ & $\begin{array}{l}-0.016 \\
(0.031)\end{array}$ \\
\hline Injury & 0.093 & 0.069 & $\begin{array}{l}-0.023 \\
(0.023)\end{array}$ & $\begin{array}{l}-0.045 \\
(0.030)\end{array}$ & 0.061 & 0.070 & $\begin{array}{c}0.010 \\
(0.016)\end{array}$ & $\begin{array}{c}0.011 \\
(0.017)\end{array}$ \\
\hline Vomitting & 0.031 & 0.116 & $\begin{array}{c}0.085^{* * *} \\
(0.019)\end{array}$ & $\begin{array}{l}0.046^{*} \\
(0.025)\end{array}$ & 0.056 & 0.057 & $\begin{array}{c}0.001 \\
(0.018)\end{array}$ & $\begin{array}{c}0.001 \\
(0.018)\end{array}$ \\
\hline Dermatological & 0.062 & 0.054 & $\begin{array}{l}-0.007 \\
(0.024)\end{array}$ & $\begin{array}{c}0.016 \\
(0.023)\end{array}$ & 0.086 & 0.070 & $\begin{array}{l}-0.016 \\
(0.021)\end{array}$ & $\begin{array}{l}-0.017 \\
(0.022)\end{array}$ \\
\hline Pregnancy & 0.037 & 0.010 & $\begin{array}{l}-0.027 \\
(0.033)\end{array}$ & $\begin{array}{c}0.013 \\
(0.018)\end{array}$ & 0.035 & 0.058 & $\begin{array}{c}0.022 \\
(0.019)\end{array}$ & $\begin{array}{c}0.024 \\
(0.019)\end{array}$ \\
\hline Pain & 0.426 & 0.346 & $\begin{array}{l}-0.080 \\
(0.081)\end{array}$ & $\begin{array}{l}-0.127 \\
(0.104)\end{array}$ & 0.648 & 0.659 & $\begin{array}{c}0.011 \\
(0.043)\end{array}$ & $\begin{array}{l}-0.008 \\
(0.037)\end{array}$ \\
\hline Number of days sick & 0.623 & 1.584 & $\begin{array}{c}0.961 \\
(4.295)\end{array}$ & $\begin{array}{l}-2.264 \\
(2.819)\end{array}$ & 1.570 & 1.742 & $\begin{array}{c}0.172 \\
(1.068)\end{array}$ & $\begin{array}{l}-0.438 \\
(1.022)\end{array}$ \\
\hline \multicolumn{9}{|l|}{ Activities of Daily Living } \\
\hline Can easily dress & 1.000 & 0.983 & $\begin{array}{c}-0.017 * * * \\
(0.006)\end{array}$ & $\begin{array}{c}-0.019 * \\
(0.009)\end{array}$ & 0.957 & 0.938 & $\begin{array}{l}-0.020 \\
(0.023)\end{array}$ & $\begin{array}{l}-0.018 \\
(0.023)\end{array}$ \\
\hline Can easily work & 0.856 & 0.901 & $\begin{array}{c}0.045 \\
(0.051)\end{array}$ & $\begin{array}{c}0.077 \\
(0.051)\end{array}$ & 0.748 & 0.798 & $\begin{array}{c}0.050 \\
(0.047)\end{array}$ & $\begin{array}{c}0.050 \\
(0.049)\end{array}$ \\
\hline Can easily lift & 0.698 & 0.730 & $\begin{array}{c}0.032 \\
(0.104)\end{array}$ & $\begin{array}{c}0.038 \\
(0.124)\end{array}$ & 0.666 & 0.692 & $\begin{array}{c}0.027 \\
(0.071)\end{array}$ & $\begin{array}{c}0.017 \\
(0.071)\end{array}$ \\
\hline Can easily walk & 0.623 & 0.699 & $\begin{array}{c}0.076 \\
(0.131)\end{array}$ & $\begin{array}{c}0.146 \\
(0.104)\end{array}$ & 0.785 & 0.755 & $\begin{array}{l}-0.030 \\
(0.074)\end{array}$ & $\begin{array}{l}-0.049 \\
(0.071)\end{array}$ \\
\hline \multicolumn{9}{|c|}{ Patient Background and Demographics } \\
\hline New patient & 0.944 & 0.850 & $\begin{array}{c}-0.094 * * \\
(0.036)\end{array}$ & $\begin{array}{l}-0.001 \\
(0.043)\end{array}$ & 0.911 & 0.903 & $\begin{array}{l}-0.008 \\
(0.037)\end{array}$ & $\begin{array}{l}-0.003 \\
(0.038)\end{array}$ \\
\hline Age & 30.006 & 25.401 & $\begin{array}{l}-4.605 \\
(3.087)\end{array}$ & $\begin{array}{l}-5.082 \\
(3.530)\end{array}$ & 28.913 & 30.700 & $\begin{array}{c}1.788 \\
(2.042)\end{array}$ & $\begin{array}{c}1.410 \\
(2.040)\end{array}$ \\
\hline Is Male & 0.494 & 0.579 & $\begin{array}{c}0.086 \\
(0.053)\end{array}$ & $\begin{array}{c}0.021 \\
(0.059)\end{array}$ & 0.487 & 0.454 & $\begin{array}{l}-0.033 \\
(0.042)\end{array}$ & $\begin{array}{l}-0.039 \\
(0.041)\end{array}$ \\
\hline Assets index & 0.455 & 0.411 & $\begin{array}{l}-0.044 \\
(0.423)\end{array}$ & $\begin{array}{l}-0.238 \\
(0.442)\end{array}$ & -0.077 & 1.006 & $\begin{array}{c}1.084^{* * *} \\
(0.220)\end{array}$ & $\begin{array}{c}1.146^{* * *} \\
(0.211)\end{array}$ \\
\hline Has formal education & 0.565 & 0.517 & $\begin{array}{l}-0.048 \\
(0.085)\end{array}$ & $\begin{array}{l}-0.053 \\
(0.081)\end{array}$ & 0.546 & 0.637 & $\begin{array}{c}0.091 * * \\
(0.035)\end{array}$ & $\begin{array}{c}0.087 * * \\
(0.034)\end{array}$ \\
\hline No. of questions patient asked & 0.369 & 0.478 & $\begin{array}{c}0.109 \\
(0.103)\end{array}$ & $\begin{array}{c}0.387^{* *} \\
(0.152)\end{array}$ & 0.488 & 0.956 & $\begin{array}{c}0.467^{* * *} \\
(0.125)\end{array}$ & $\begin{array}{c}0.472^{* * *} \\
(0.125)\end{array}$ \\
\hline Is from this village & 0.759 & 0.529 & $\begin{array}{c}-0.230 * * * \\
(0.060)\end{array}$ & $\begin{array}{c}-0.149 * * \\
(0.063)\end{array}$ & 0.538 & 0.582 & $\begin{array}{c}0.045 \\
(0.049)\end{array}$ & $\begin{array}{c}0.036 \\
(0.051)\end{array}$ \\
\hline Came by foot & 0.741 & 0.451 & $\begin{array}{c}-0.290 * * * \\
(0.044)\end{array}$ & $\begin{array}{c}-0.158^{* * *} \\
(0.041)\end{array}$ & 0.594 & 0.414 & $\begin{array}{c}-0.180^{* *} \\
(0.068)\end{array}$ & $\begin{array}{c}-0.186^{* * *} \\
(0.068)\end{array}$ \\
\hline
\end{tabular}

Notes: *** Significant at $1 \%, * *$ Significant at $5 \%, *$ Significant at $10 \%$. Robust standard errors clustered at the market level are in parenthesis. Data is from patient-exit surveys which obtained by observing all providers for a full day of practice. Columns (3) and (7) present binary regression coefficients from estimating the relevant row variable on an indicator for private provider visit, and thus represent the mean difference of the row variable between private and public sectors. Columns (4) and (8) repeat the exercise but add market fixed effects in the representative sample and district fixed effects in the dual sample. 
Table A.15: Difference between dual and non-dual providers' treatment of SPs (public sample only)

\begin{tabular}{|c|c|c|c|c|c|c|c|c|c|c|c|c|c|}
\hline & (1) & (2) & (3) & (4) & (5) & (6) & (7) & (8) & (9) & (10) & (11) & (12) & (13) \\
\hline & \multicolumn{3}{|c|}{ Effort } & \multicolumn{3}{|c|}{ Diagnosis } & \multicolumn{7}{|c|}{ Treatment } \\
\hline & Time spent & Checklist & IRT Score & $\begin{array}{c}\text { Gave } \\
\text { diagnosis }\end{array}$ & $\begin{array}{c}\text { Correct } \\
\text { diagnosis } \\
\text { (conditional) }\end{array}$ & $\begin{array}{c}\text { Correct } \\
\text { diagnosis } \\
\text { (unconditional) }\end{array}$ & $\begin{array}{l}\text { Correct } \\
\text { treatment }\end{array}$ & $\begin{array}{l}\text { Palliative } \\
\text { treatment }\end{array}$ & $\begin{array}{c}\text { Unnecessary } \\
\text { treatment }\end{array}$ & $\begin{array}{c}\text { Correct } \\
\text { treatment } \\
\text { Only }\end{array}$ & Antibiotic & $\begin{array}{c}\text { Number of } \\
\text { medicines }\end{array}$ & $\begin{array}{c}\text { Referred } \\
\text { patient }\end{array}$ \\
\hline \multicolumn{14}{|c|}{ Panel A: Dual practice sample, with SP, case and district fixed effects } \\
\hline Is a dual provider & $\begin{array}{c}-0.659 * * * \\
(0.210)\end{array}$ & $\begin{array}{l}-3.881^{*} \\
(2.045)\end{array}$ & $\begin{array}{l}-0.329 \\
(0.218)\end{array}$ & $\begin{array}{l}-0.022 \\
(0.057)\end{array}$ & $\begin{array}{c}0.021 \\
(0.100)\end{array}$ & $\begin{array}{c}0.014 \\
(0.034)\end{array}$ & $\begin{array}{l}-0.007 \\
(0.064)\end{array}$ & $\begin{array}{l}-0.029 \\
(0.072)\end{array}$ & $\begin{array}{l}-0.029 \\
(0.064)\end{array}$ & $\begin{array}{l}-0.017 \\
(0.026)\end{array}$ & $\begin{array}{l}-0.106 \\
(0.077)\end{array}$ & $\begin{array}{l}-0.212 \\
(0.209)\end{array}$ & $\begin{array}{l}-0.015 \\
(0.049)\end{array}$ \\
\hline R-squared & 0.268 & 0.131 & & 0.187 & 0.319 & 0.113 & 0.340 & 0.211 & 0.096 & 0.044 & 0.142 & 0.153 & 0.159 \\
\hline Number of observations & 252 & 252 & 103 & 252 & 73 & 252 & 161 & 161 & 161 & 161 & 161 & 161 & 161 \\
\hline Mean of non-dual observations & 2.343 & 22.788 & & 0.326 & 0.207 & 0.067 & 0.295 & 0.705 & 0.836 & 0.033 & 0.541 & 2.885 & 0.131 \\
\hline Mean of dual observations & 1.562 & 17.677 & & 0.270 & 0.341 & 0.092 & 0.380 & 0.630 & 0.830 & 0.020 & 0.480 & 2.800 & 0.080 \\
\hline Mean of sample & 1.838 & 19.482 & & 0.290 & 0.288 & 0.083 & 0.348 & 0.658 & 0.832 & 0.025 & 0.503 & 2.832 & 0.099 \\
\hline \multicolumn{14}{|c|}{ Panel B: Dual practice sample, with SP, case and district fixed effects } \\
\hline Is a dual provider & $\begin{array}{c}-0.677 * * * \\
(0.248)\end{array}$ & $\begin{array}{l}-4.285^{*} \\
(2.291)\end{array}$ & $\begin{array}{c}-0.420^{*} \\
(0.253)\end{array}$ & $\begin{array}{l}-0.065 \\
(0.071)\end{array}$ & $\begin{array}{l}-0.092 \\
(0.140)\end{array}$ & $\begin{array}{l}-0.020 \\
(0.042)\end{array}$ & $\begin{array}{l}-0.007 \\
(0.077)\end{array}$ & $\begin{array}{l}-0.005 \\
(0.090)\end{array}$ & $\begin{array}{l}-0.073 \\
(0.073)\end{array}$ & $\begin{array}{l}-0.012 \\
(0.029)\end{array}$ & $\begin{array}{c}-0.153^{*} \\
(0.088)\end{array}$ & $\begin{array}{l}-0.293 \\
(0.267)\end{array}$ & $\begin{array}{l}-0.050 \\
(0.060)\end{array}$ \\
\hline Age of provider & $\begin{array}{c}-0.017 * \\
(0.009)\end{array}$ & $\begin{array}{l}-0.077 \\
(0.101)\end{array}$ & $\begin{array}{l}-0.001 \\
(0.011)\end{array}$ & $\begin{array}{l}-0.001 \\
(0.003)\end{array}$ & $\begin{array}{l}-0.003 \\
(0.008)\end{array}$ & $\begin{array}{l}-0.002 \\
(0.002)\end{array}$ & $\begin{array}{l}-0.004 \\
(0.004)\end{array}$ & $\begin{array}{l}-0.007 \\
(0.004)\end{array}$ & $\begin{array}{c}0.001 \\
(0.003)\end{array}$ & $\begin{array}{l}-0.002 \\
(0.001)\end{array}$ & $\begin{array}{l}-0.006 \\
(0.004)\end{array}$ & $\begin{array}{c}-0.028^{* *} \\
(0.012)\end{array}$ & $\begin{array}{c}0.000 \\
(0.002)\end{array}$ \\
\hline Patient load during visit & $\begin{array}{l}-0.031 \\
(0.040)\end{array}$ & $\begin{array}{c}0.495 \\
(0.584)\end{array}$ & $\begin{array}{c}0.036 \\
(0.031)\end{array}$ & $\begin{array}{c}0.008 \\
(0.011)\end{array}$ & $\begin{array}{l}-0.019 \\
(0.048)\end{array}$ & $\begin{array}{l}-0.000 \\
(0.010)\end{array}$ & $\begin{array}{l}-0.010 \\
(0.019)\end{array}$ & $\begin{array}{c}0.033 * * \\
(0.016)\end{array}$ & $\begin{array}{c}0.004 \\
(0.023)\end{array}$ & $\begin{array}{l}-0.006 \\
(0.005)\end{array}$ & $\begin{array}{c}0.002 \\
(0.026)\end{array}$ & $\begin{array}{c}0.006 \\
(0.086)\end{array}$ & $\begin{array}{c}0.020 \\
(0.017)\end{array}$ \\
\hline R-squared & 0.310 & 0.143 & & 0.198 & 0.375 & 0.145 & 0.360 & 0.259 & 0.202 & 0.099 & 0.255 & 0.248 & 0.275 \\
\hline Number of observations & 215 & 215 & 90 & 215 & 62 & 215 & 137 & 137 & 137 & 137 & 137 & 137 & 137 \\
\hline
\end{tabular}

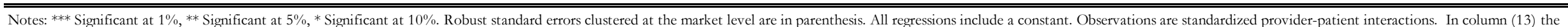
dependent variable is total number of medicines recommended to the patient (dispensed and/or prescribed). 
Table A.16: Robustness to alternative metrics for public-private comparison

\begin{tabular}{|c|c|c|c|c|c|c|c|c|c|c|c|}
\hline & (1) & (2) & (3) & (4) & (5) & (6) & (7) & (8) & (9) & (10) & (11) \\
\hline & \multicolumn{2}{|c|}{ Effort } & \multicolumn{3}{|c|}{ Diagnosis } & \multicolumn{6}{|c|}{ Treatment } \\
\hline & Time spent & Checklist & Gave diagnosis & $\begin{array}{c}\text { Correct } \\
\text { diagnosis } \\
\text { (conditional) }\end{array}$ & $\begin{array}{c}\text { Correct } \\
\text { diagnosis } \\
\text { (unconditional) }\end{array}$ & $\begin{array}{l}\text { Correct } \\
\text { treatment }\end{array}$ & $\begin{array}{l}\text { Palliative } \\
\text { treatment }\end{array}$ & $\begin{array}{c}\text { Unnecessary } \\
\text { treatment }\end{array}$ & $\begin{array}{l}\text { Correct } \\
\text { treatment } \\
\text { only }\end{array}$ & Antibiotic & $\begin{array}{c}\text { Number of } \\
\text { medicines }\end{array}$ \\
\hline \multicolumn{12}{|c|}{ Panel A: Best public vs. best private (by correct treatment) } \\
\hline Is a private provider & $\begin{array}{c}1.632^{* * *} \\
(0.388)\end{array}$ & $\begin{array}{c}11.288^{* * *} \\
(2.855)\end{array}$ & $\begin{array}{c}0.235^{* * *} \\
(0.090)\end{array}$ & $\begin{array}{c}0.033 \\
(0.136)\end{array}$ & $\begin{array}{c}0.079 \\
(0.054)\end{array}$ & $\begin{array}{c}0.162^{* *} \\
(0.079)\end{array}$ & $\begin{array}{c}0.074 \\
(0.077)\end{array}$ & $\begin{array}{c}0.169 \\
(0.117)\end{array}$ & $\begin{array}{l}-0.014 \\
(0.056)\end{array}$ & $\begin{array}{c}0.143 \\
(0.109)\end{array}$ & $\begin{array}{c}1.147^{* * *} \\
(0.429)\end{array}$ \\
\hline R-squared & 0.453 & 0.417 & 0.430 & 0.714 & 0.363 & 0.592 & 0.447 & 0.353 & 0.218 & 0.435 & 0.463 \\
\hline Number of observations & 286 & 286 & 192 & 76 & 192 & 192 & 192 & 192 & 192 & 192 & 192 \\
\hline Mean of public & 2.547 & 16.000 & 0.271 & 0.154 & 0.042 & 0.271 & 0.521 & 0.708 & 0.042 & 0.250 & 2.063 \\
\hline Mean of private & 3.613 & 24.551 & 0.438 & 0.238 & 0.104 & 0.438 & 0.535 & 0.750 & 0.049 & 0.292 & 3.014 \\
\hline Mean of sample & 3.352 & 22.458 & 0.396 & 0.224 & 0.089 & 0.396 & 0.531 & 0.740 & 0.047 & 0.281 & 2.776 \\
\hline \multicolumn{12}{|c|}{ Panel B: Best public vs. best private (by checklist items) } \\
\hline Is a private provider & $\begin{array}{c}3.216^{* * *} \\
(0.916)\end{array}$ & $\begin{array}{c}16.987^{* * *} \\
(5.003)\end{array}$ & $\begin{array}{c}0.263^{* *} \\
(0.116)\end{array}$ & $\begin{array}{c}0.119 \\
(0.160)\end{array}$ & $\begin{array}{l}0.079 \\
(0.056)\end{array}$ & $\begin{array}{c}0.141 \\
(0.095)\end{array}$ & $\begin{array}{c}0.034 \\
(0.104)\end{array}$ & $\begin{array}{c}0.167 \\
(0.139)\end{array}$ & $\begin{array}{l}-0.027 \\
(0.028)\end{array}$ & $\begin{array}{c}0.222 \\
(0.156)\end{array}$ & $\begin{array}{c}1.581^{* * *} \\
(0.503)\end{array}$ \\
\hline R-squared & 0.586 & 0.501 & 0.610 & 0.823 & 0.487 & 0.616 & 0.699 & 0.468 & 0.540 & 0.473 & 0.674 \\
\hline Number of observations & 191 & 191 & 129 & 63 & 129 & 129 & 129 & 129 & 129 & 129 & 129 \\
\hline Mean of public & 2.481 & 18.832 & 0.333 & 0.133 & 0.044 & 0.200 & 0.556 & 0.689 & 0.022 & 0.178 & 1.800 \\
\hline Mean of private & 4.708 & 30.269 & 0.571 & 0.146 & 0.083 & 0.286 & 0.595 & 0.845 & 0.012 & 0.310 & 3.381 \\
\hline Mean of sample & 3.938 & 26.317 & 0.488 & 0.143 & 0.070 & 0.256 & 0.581 & 0.791 & 0.016 & 0.264 & 2.829 \\
\hline
\end{tabular}

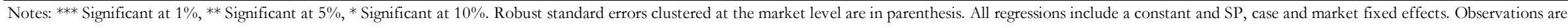
standardized provider-patient interactions. In column (11) the dependent variable is total number of medicines recommended to the patient (dispensed and/or prescribed). 